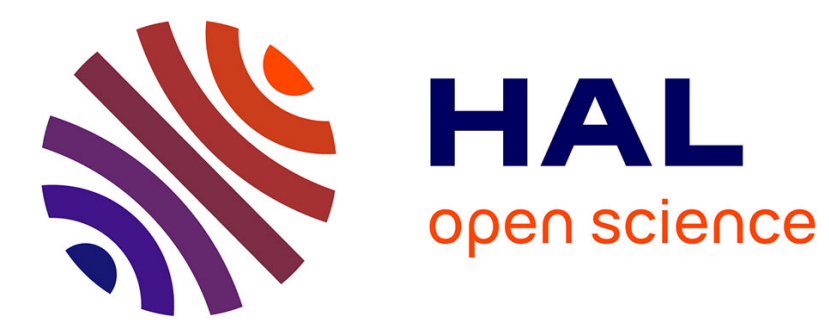

\title{
Deep learning massively accelerates super-resolution localization microscopy
}

Wei Ouyang, Andrey Aristov, Mickaël Lelek, Xian Hao, Christophe Zimmer

\section{To cite this version:}

Wei Ouyang, Andrey Aristov, Mickaël Lelek, Xian Hao, Christophe Zimmer. Deep learning massively accelerates super-resolution localization microscopy. Nature Biotechnology, 2018, 36 (5), pp.460-468. 10.1038/nbt.4106 . pasteur-02074397

\section{HAL Id: pasteur-02074397}

\section{https://hal-pasteur.archives-ouvertes.fr/pasteur-02074397}

Submitted on 5 Mar 2020

HAL is a multi-disciplinary open access archive for the deposit and dissemination of scientific research documents, whether they are published or not. The documents may come from teaching and research institutions in France or abroad, or from public or private research centers.
L'archive ouverte pluridisciplinaire HAL, est destinée au dépôt et à la diffusion de documents scientifiques de niveau recherche, publiés ou non, émanant des établissements d'enseignement et de recherche français ou étrangers, des laboratoires publics ou privés. 
2 ANNA-PALM: Deep learning accelerates super-resolution localization

3 microscopy by orders of magnitude

4

5

6 Wei Ouyang $1,2,3$, Andrey Aristov ${ }^{1,2,3}$, Mickaël Lelek ${ }^{1,2,3}$, Xian Hao ${ }^{1,2,3}$, Christophe

$7 \quad$ Zimmer ${ }^{1,2,3}$

8

$9 \quad{ }^{1}$ Institut Pasteur, Unité Imagerie et Modélisation, Paris, France

$10 \quad 2$ UMR 3691, CNRS

$11{ }^{3}$ C3BI, USR 3756, IP CNRS

12

13

14

15

16

17

18

19

20

21

22

23 Corresponding author: C. Zimmer 
24 The speed of super-resolution microscopy methods based on single molecule

25 localization, e.g. PALM or STORM, is severely limited by the need to record

26 many thousands of frames with a low number of observed molecules in each.

27 Here, we present ANNA-PALM, a computational strategy that uses artificial

28 neural networks to reconstruct super-resolution views from sparse, rapidly

29 acquired localization images and/or widefield images. Simulations and

30 experimental imaging of microtubules, nuclear pores and mitochondria show

31 that high-quality super-resolution images can be reconstructed from up to two

32 orders of magnitude fewer frames than usually needed, without

33 compromising spatial resolution. Super-resolution reconstructions are even

34 possible from widefield images alone, though adding localization data

35 improves image quality. We demonstrate super-resolution imaging of $>\mathbf{1 , 0 0 0}$

36 fields of view containing $>1,000$ cells in $\sim 3 \mathrm{~h}$, yielding an image spanning

37 spatial scales from $\sim 20 \mathrm{~nm}$ to $\sim 2 \mathrm{~mm}$. The drastic reduction in acquisition

38 time and sample irradiation afforded by ANNA-PALM enables faster and

39 gentler high-throughput and live cell super-resolution imaging. 
41 Fluorescence microscopy methods that overcome the diffraction limit of resolution

42 ( 200-300 nm) allow imaging of biological structures with molecular specificity 43 closer to the molecular scale. Among super-resolution microscopy approaches,

44 those based on single molecule localization, such as PALM$^{1}$ or STORM$^{2}$ (hereafter 45 referred to collectively as PALM) are particularly attractive owing to their exquisite 46 spatial resolution and ease of implementation. In these methods, random subsets of 47 fluorophores are imaged in many consecutive diffraction-limited frames, 48 computationally localized to high precision, and the combined localizations are used 49 to generate a super-resolution view. In practice, typically $10^{3}-10^{5}$ diffraction-limited

50 frames are needed to assemble a single super-resolution image. This requirement

51 follows from two conditions that must be simultaneously satisfied to ensure high

52 spatial resolution: (i) a low number $\left(\sim 10-10^{2}\right)$ of active fluorophores per frame, to

53 avoid overlaps between diffraction limited spots and enable precise localization of

54 individual molecules, and (ii) a large number of independent localizations to ensure

55 a sufficiently dense sampling of the underlying biological structures ${ }^{3,4}$. The large

56 number of required frames makes localization microscopy inherently slow, thereby

57 limiting its potential for high-throughput imaging, where many fields of view (FoVs)

58 are to be imaged, and for imaging live cell dynamics. As a result, most localization

59 microscopy studies are restricted to analyzing a small number of cells (typically less

60 than ten).

61 Multiple approaches have been explored to accelerate localization

62 microscopy. Using bright dyes with rapid switching kinetics, high power lasers and

63 fast cameras allows to minimize exposure time without losing signal to noise 
64 ratio 5,6 , but reaching sub-millisecond exposure remains challenging, and intense

65 irradiation exacerbates phototoxicity in live cell imaging ${ }^{7,8}$. Increasing the number

66 of active fluorophores per frame can reduce acquisition time, but despite algorithms

67 designed to handle overlapping fluorescent spots $^{9-13}$ this approach necessarily

68 compromises spatial resolution ${ }^{14,15}$.

69 Here, we introduce a computational strategy that allows reducing the total

70 number of frames and independent localizations without losing spatial resolution:

71 Unlike previous approaches, our method leverages the structural redundancy of

72 most biological images to reconstruct high quality images from vastly under-

73 sampled localization microscopy data. Our method leverages deep learning, which

74 employs artificial neural networks (ANNs) to learn complex non-linear mappings

75 between numerical inputs and outputs ${ }^{16}$. Accordingly, we call it 'artificial neural

76 network accelerated PALM', or ANNA-PALM.

\section{RESULTS}

79 A deep learning approach to super-resolution image reconstruction

80 We aim to reconstruct a super-resolution image of approximately similar 81 information content as a standard PALM acquisition (with $K$ frames and $N$

82 localizations) from a much smaller number of raw frames $(k<<K)$ without changing

83 the average density of localizations, $\rho$, i.e. from a much smaller number of total

84 localizations $(n=\rho k \ll N=\rho K)$. If PALM images are defined as 2D histograms of

85 independent localizations, this task can be formulated as restoring an image

86 corrupted by Poisson noise (and potentially additional forms of noise). Image 
87 restoration is an ill-posed problem that has an infinity of solutions in the high-

88 dimensional space of all possible images, unless additional constraints (priors) are

89 imposed that restrict the solution to a lower dimensional subspace. Suitable

90 subspaces exist because most natural images are highly redundant, and can be

91 represented to very good approximation with a much smaller number of coefficients

92 than pixels, via appropriate functions that map feature space to pixel space ${ }^{17,18}$. In

93 recent years, ANNs with multiple layers (deep nets) have proven very successful at

94 learning meaningful features and non-linear mappings for image classification,

95 segmentation, restoration and many other tasks ${ }^{16,18,19}$. Inspired by these

96 developments, we designed ANNA-PALM, a deep learning approach for restoring

97 super-resolution views from under-sampled (sparse) localization microscopy data.

98 ANNA-PALM comprises a training stage and an inference stage (Figure 1).

99 For training (Figure 1a), a few super-resolution images representative of the

100 structure of interest (e.g. microtubules, nuclear pores, or mitochondria) are

101 obtained using standard PALM imaging, i.e. by acquiring long diffraction limited

102 image sequences (e.g. $K \sim 10^{4}-10^{5}, N \sim 10^{5}-10^{7}$ ) and processing them with

103 standard localization software ${ }^{20}$, resulting in highly sampled (dense) PALM images.

104 In addition, a low resolution (widefield) image can also be acquired, as is commonly

105 done before single molecule imaging when bleaching out preactivated fluorophores.

106 Next, the dense PALM images are under-sampled by using a much smaller number

107 of input frames, $k \ll K$, thus yielding sparse PALM images from the same

108 localization data. Then, an ANN is trained to recover approximations of the dense

109 PALM images from these sparse PALM images (and the optional widefield image). 
110 Once trained, the ANN is applied to new sparse PALM images (with or without a

111 widefield image), obtained from new image sequences with small numbers of

112 frames $(k \ll K)$-and hence in much shorter time- in order to reconstruct high

113 quality super-resolution images not previously seen (inference, Figure 1b).

\section{Neural net architecture and learning strategy}

116 Our ANN, hereafter called A-net, contains a total of 25 convolutional layers, and

117 roughly 42 million trainable parameters. A-net is adapted from the pix2pix

118 network ${ }^{21}$, which itself builds on U-nets ${ }^{22}$ and generative adversarial networks

119 (GANs) ${ }^{23}$, two recent successful deep learning techniques. U-nets are special types of

120 convolutional neural networks (CNNs) that have proven effective at learning multi-

121 scale representations of images and accurate, pixel-wise mappings ${ }^{22,24}$. GANs can

122 generate new samples from real image distributions using a generator network that

123 outputs synthetic images, and a discriminator network that outputs the probability

124 that an input image is real or synthetic, both networks being trained simultaneously

125 to compete against each other ${ }^{23}$. Importantly, the generator can be conditioned on

126 input data (conditional GAN, or cGAN) ${ }^{21,23}$, e.g. on images as in the pix2pix network.

127 We modified the pix2pix architecture to accept a computational switch as additional

128 input to handle multiple types of data, and introduced an additional network to

129 evaluate the consistency between the reconstructed image and the widefield input

130 image.

131 Training of our A-net proceeds as follows. Randomly under-sampled (i.e.

132 sparse) versions of PALM images are fed as input to the A-net, while the 
133 corresponding dense PALM images are defined as the A-net's targets, i.e. desired

134 outputs (Figure 1a). Additional, optional inputs are widefield images, if available,

135 and the switch, which indicates the image type when multiple types of images (e.g.

136 microtubules and nuclear pores) are used during training. ANN training requires

137 defining an objective function (also called loss), which measures how well the

138 outputs match the targets. We implemented a loss function containing three terms.

139 The first term measures the difference between the A-net output and the dense

140 PALM image. Instead of the widely used mean squared error (MSE), which poorly

141 reflects visual quality25, we used a combination of the absolute difference (L1 norm)

142 with a multi-scale version of the structural similarity index, a perceptually

143 motivated quality metric shown to improve image restoration with deep learning

144 (MS-SSIM) ${ }^{26}$. The second term measures the consistency between the A-net output

145 and the widefield image. Although in theory the latter should simply be a blurred

146 version of the PALM image, this is often not the case in practice ${ }^{27}$. Therefore, we

147 introduced another CNN (with 4-layers), called 'low resolution estimator' to predict

148 the widefield image from the super-resolution image. The corresponding loss was

149 defined as the MS-SSIM between this CNN's output and the observed widefield

150 image. (In absence of a widefield image, this loss is set to zero). The third term

151 contains a cGAN discriminator $\operatorname{loss}^{21}$, where the discriminator is a 5-layer CNN,

152 whose inputs are the sparse PALM (and widefield) image(s) and either the dense

153 PALM image or the output of the generator above; the discriminator's output is

154 compared to 0 s and 1 s (for synthetic and real, respectively), respectively, via the

155 MSE. We use dropout ${ }^{28}$ and extensive data augmentation, including random 
156 rotations, translations, elastic deformations and addition of noise in the input image

157 to mimic false detections and unspecific labeling. As a result, only a few dense PALM

158 images are required for successful training without overfitting. On graphical

159 processing units (GPU), training ANNA-PALM from scratch takes on the order of

160 hours to days, but when starting from a previously trained A-net, retraining can be

161 done in an hour or less.

162 Once trained, the A-net can take sparse localization data with an optional

163 widefield image as input(s), and output a reconstructed super-resolution image in

164 less than a second (Figure 1b). In addition, the A-net produces an 'error map' that

165 measures the consistency of this super-resolution image with the widefield image ${ }^{27}$

166 (when available) and can be used to estimate the degree of reliability and highlight

167 potential reconstruction artifacts. For more details, see Online Methods and

\section{Supplementary Note 1.}

\section{Validating ANNA-PALM on simulated images}

171 We first sought to validate ANNA-PALM on synthetic data. For this, we used

172 Brownian dynamics simulations ${ }^{29}$ to generate 200 dense PALM images of semi-

173 flexible filaments mimicking microtubules, with a resolution $R_{\text {loc }} \approx 23 \mathrm{~nm}$. These

174 represent "perfect" PALM images that would be obtained with an infinite number of

175 localizations. We applied varying levels of Poisson noise to these perfect images to

176 create sparse PALM images corresponding to finite numbers of localizations

177 (Supplementary Figure 1). We then trained our A-net using the perfect images as 
178 targets and the sparse images (and widefield image) as inputs, varying the number

179 of localizations over a large range.

180 Next, we applied the trained A-net to a distinct set of PALM images generated

181 by the same stochastic simulation (Supplementary Figure 2). Figure 2a shows a

182 widefield image and Figure $\mathbf{2 b}$ a corresponding sparse PALM image obtained from

$183 n=6,834$ localizations. Although curvilinear structures can be seen in this image

184 despite its sparsity, small-scale features remain highly ambiguous (Figure $\mathbf{2 b}$,

185 inset), and the resolution according to a recently proposed five-fold Nyquist

186 criterion $^{3}$ is limited by sampling to $R_{5 \times \mathrm{Nyq}} \approx 85 \mathrm{~nm}$; according to this criterion,

$187 N>N_{5 \times N y q}=60,000$ localizations are needed to achieve $23 \mathrm{~nm}$ resolution

188 (Supplementary Figure 3d). Figure 2c shows the ANNA-PALM image

189 reconstructed from the wide-field image alone, which exhibits clear and continuous

190 filaments that were not previously recognizable. Most of the relatively isolated

191 filaments roughly agree with the perfect PALM image (Figure 2e). In the denser

192 regions, however, many small features are erroneous, e.g. filaments are incorrectly

193 joined, displaced, split or merged (Figure 2c, blue arrows and Supplementary

194 Figure 4, top). By contrast, the ANNA-PALM image reconstructed from the sparse

195 PALM image alone or in combination with the widefield image exhibits continuous

196 and sharp filaments in very good agreement with the perfect PALM image

197 (Supplementary Figure 3b,c and Figure 2d,e,f). The spatial resolution of these

198 reconstructed images is limited neither by diffraction nor sampling, but only by the

199 localization precision, and is thus $R_{\text {loc }} \approx 23 \mathrm{~nm}$, as in the perfect images

200 (Supplementary Figure 3e,f). These results indicate that high quality super- 
201 resolution images can be obtained from only a small fraction of the number of 202 localizations traditionally required (here, approximately $\sim 11 \%$ of $N_{5 \times N y q}$ above; see 203 Supplementary Figure 3d), hence enabling a strong reduction in acquisition time. 204 Nevertheless, reconstruction errors can still occur in areas where the sparse 205 localization data are most ambiguous, e.g. where filament density is highest 206 (Figure 2d,e,f, white arrow). These errors can be reduced by increasing the 207 localization number $n$, implying a trade-off between acquisition time and 208 reconstruction quality (Supplementary Figure 4).

209 To quantify this trade-off, we computed the MS-SSIM between reconstructed 210 ANNA-PALM and perfect PALM images $(n=\infty)$ as function of localization number, 211 from $n \sim 200$ to $n \sim 2 \times 10^{6}$, in comparison with the standard PALM images 212 (Figure 2g). The MS-SSIM ranges from 0 to 1 and reaches 1 for perfect 213 reconstructions. For standard PALM images, the MS-SSIM increases monotonically, 214 as expected, from $<0.2$ to $>0.95$ for $n=2 \times 10^{6}$ million localizations (Figure 2g, black 215 curve). Using only the sparse image as input, ANNA-PALM reconstructions achieve 216 MS-SSIM that are consistently higher and increase with localization number $n$ much 217 more rapidly than standard PALM, already exceeding 0.9 for $n \approx 10,000$ 218 localizations (Figure 2, dashed blue curve). ANNA-PALM achieves the same MS219 SSIM as standard PALM at the five-fold Nyquist sampling level $(\approx 0.65)$ with only $220 n=2,248$ localizations instead of $n=58,588$, suggesting a $\sim 26$-fold speed-up. If the 221 widefield image is used as additional input, the MS-SSIM further increases, and 222 dramatically so for low localization numbers (Figure 2g, solid blue curve). For 223 example, with $n=7,477$ localizations, ANNA-PALM achieves a MS-SSIM $(\approx 0.95)$ 
224 similar to standard PALM with $n=644,844$, implying a speed-up of roughly two

225 orders of magnitude. (Note that, if the perfect PALM image was not available for

226 these quantifications, it could be replaced by the ANNA-PALM reconstruction of a

227 dense PALM image with a large number of localizations, e.g. $n=10^{5}$, with similar

228 results- see Supplementary Figure 5).

229 As any image restoration method, ANNA-PALM can make errors. The low

230 resolution error map described above (Figure 1b) provides a means to estimate

231 where errors are most likely to occur. When applied to ANNA-PALM reconstructions

232 of a sparse PALM image, this error map highlights regions containing the highest

233 density of filament crossings, where reconstructions tend to be least accurate

234 (Supplementary Figure 6i,k). If we artificially displace a small piece of filament in

235 this image to simulate a false positive and a false negative in the reconstruction

236 (Supplementary Figure 6b,d, white and blue arrows, respectively), the affected

237 regions also light up in the error map (Supplementary Figure 6j,1). Thus, the error

238 map offers a useful tool to highlight regions most likely to contain reconstruction

239 errors, and conversely, to outline regions where reconstructions are most

240 trustworthy. Thus, simulations suggest that ANNA-PALM can considerably reduce

241 acquisition time in localization microscopy and also map reconstruction reliability.

\section{ANNA-PALM reconstructions of immunostained microtubules}

244 We next tested our method on real images of immunolabeled microtubules

245 (Figure 3). We trained our A-net on seven dense PALM images (with corresponding

246 widefield images) obtained during 10 minute long acquisitions $(K=60,000$; 
$247 \Delta \mathrm{t}=10 \mathrm{~ms}$ exposure time) (not shown). We then considered a sparse PALM image of

248 microtubules in a distinct FoV obtained from only $9 \mathrm{~s}$ of acquisition $(k=300 ; \Delta \mathrm{t}=30$

$249 \mathrm{~ms})$, together with a widefield image $(\Delta \mathrm{t}=2 \times 50 \mathrm{~ms}$ ) (Figure 3a,b). Whereas

250 microtubule filaments can already be seen in this sparse PALM image, structural

251 details below the diffraction limit are hard to discern, making it difficult to follow

252 the path of individual filaments in the denser regions and to identify features such

253 as filament crossings (Figure 3b). By contrast, the ANNA-PALM images, whether

254 reconstructed from the widefield image alone, the sparse PALM image alone, or

255 both, all display sharp and continuous filaments and clearly reveal many structural

256 details (Figure 3d-f). Their resolution is similar to or even better than the dense

257 PALM image (Supplementary Figure 7a). As for the simulations, in regions where

258 microtubule filaments are isolated, the ANNA-PALM image reconstructed from the

259 widefield image alone is in good agreement with the dense PALM image

260 (Figure 3d,g). However, it is often incorrect in areas of high microtubule density

261 (e.g. Figure 3d,g white and gray arrows). Most of these reconstruction errors are

262 corrected when applying ANNA-PALM to the sparse PALM image instead

263 (Figure 3e,h). For example, parallel sections of two microtubules unresolved in the

264 widefield image and incorrectly merged in Figure 3d are now clearly separated and

265 positioned correctly, and missed portions of other filaments are now recovered

266 (Figure 3h, white and gray arrows). Counter-intuitively, the sparse PALM image

267 exhibits high signal in some locations where the dense PALM image does not,

268 presumably because of spurious localizations due e.g. to unspecific binding

269 (Figure 3b, blue arrow). Such signal can lead to incorrect features in the ANNA- 
270 PALM reconstruction from the sparse localization data alone (Figure 3e,h, blue 271 arrows). However, when combining the widefield and sparse PALM data, these 272 artifacts are largely removed and reconstructions agree very well with the dense 273 PALM image (Figure 3f,i). Reconstruction quality increases with the number of 274 frames $k$ (Figure 3j, Supplementary Figures 8-9 and Supplementary Video 1). 275 More quantitatively, a MS-SSIM analysis similar to that for the simulated data above 276 (with the ANNA-PALM output of the dense PALM image defined as ground truth; see 277 Supplementary Figures 5, 9f) suggests that ANNA-PALM allows a hundred-fold 278 reduction of acquisition time compared to standard PALM (Supplementary 279 Figure 9g). Supplementary Figure 10 shows other examples of sparse 280 microtubule images reconstructed by ANNA-PALM.

281 As for simulations above, we used the widefield image to compute an error 282 map (Supplementary Figures 6,11). Bright areas in this error map highlight 283 regions where the reconstruction indeed disagrees with the dense PALM image; 284 conversely, reconstructions are of high quality in the majority of regions where the 285 error map is dim (Supplementary Figure 11d-f). These results demonstrate 286 experimentally that ANNA-PALM can restore high quality approximations of super287 resolution images from much shorter acquisition time than typical for PALM 288 imaging, and also predict where reconstruction errors are most likely.

290 ANNA-PALM enables high-throughput super-resolution imaging

291 The drastic improvement in imaging efficiency afforded by ANNA-PALM permits 292 super-resolution imaging of orders of magnitude more cells and FoVs per unit time. 
293 To demonstrate this, we used an automated acquisition protocol to image $>1,000$

294 cells with immunolabeled microtubules in 1,089 (33x33), partly overlapping, FoVs

295 of $55.3 \mu \mathrm{m} \times 55.3 \mu \mathrm{m}$ each (Figure 4, Supplementary Figure 12). We first acquired

296 widefield images at each of these positions, in a total of $\sim 12$ minutes, mostly

297 consisting of stage stabilization delays (Supplementary Figure 12a). Next, we

298 obtained 1,089 sparse PALM images using only $10 \mathrm{~s}$ of imaging time per FoV

299 ( $k=1,000$ frames, $\Delta t=10 \mathrm{~ms}$ ), in a total of only $\sim 3.1$ hours (Figure 4a). Neither the

300 widefield nor the sparse PALM images provided much small scale information

301 (Figure 4c, Supplementary Figure 12 c,e). However, ANNA-PALM reconstructions

302 led to high quality super-resolution images, allowing to visualize the microtubule

303 network with clarity and to distinguish microtubule filaments in dense areas that

304 appeared as unstructured regions in the sparse PALM image (Figure $\mathbf{4 b}, \mathbf{d}$ ). The

305 FWHM across filaments in the reconstructed image was $\sim 51 \mathrm{~nm}$ (Figure 4d), within

306 the range measured for the training data (Supplementary Figure 7a). Similar

307 images can be obtained by ANNA-PALM using the widefield images alone

308 (Supplementary Figure 12), although of lower quality, as discussed above.

309 Stitching the reconstructed images together yielded a single super-resolution image

310 that contained approximately seven billion $20 \times 20 \mathrm{~nm}$ pixels and covered an area of

$3111.8 \mathrm{~mm} \times 1.8 \mathrm{~mm}$, thereby spanning almost five orders of magnitude in spatial scales

312 (Figure 4b,d, Supplementary Figure 12b and Supplementary Video 2).

313

314 ANNA-PALM is robust to experimental perturbations 
315 ANNA-PALM can reconstruct accurate super-resolution images from sparse data 316 because it was trained on similar images before. This raises the question of whether 317 an ANN trained in one experimental condition can be successfully applied to 318 another condition. To test this, we used the A-net as trained above to analyze

319 microtubule images of cells subjected to drugs affecting the cytoskeletal network.

320 We first treated U373 cells with $1 \mu \mathrm{M}$ of Taxol, an antimitotic agent that inhibits the

321 depolymerization of microtubules and increases their bending rigidity ${ }^{30,31}$.

322 Figure $5 \mathbf{d}$ shows a sparse PALM image of these cells $(k=800, \mathrm{k} \Delta \mathrm{t}=8 \mathrm{~s})$, in which the 323 microtubule network is barely recognizable. By contrast, the ANNA-PALM

324 reconstructions clearly display a complex microtubule network and agree well with 325 the dense PALM image obtained from $K=60,000$ frames $(K \Delta t=10 \mathrm{~min})$

326 (Figure 5d,e,f). These images display a larger density of straighter and more 327 parallel filaments with less frequent crossings than in the untreated cells 328 (Figure 5a-c), consistent with microtubule stabilization and increased rigidity ${ }^{31}$.

329 Next, we treated cells with $1 \mu \mathrm{M}$ of Nocodazole, a drug that, on the opposite, 330 promotes microtubule depolymerization and is expected to more dramatically alter 331 the cytoskeletal network ${ }^{32}$. Again, whereas the sparse image obtained from $k=300$ 332 frames $(k \Delta t=3 \mathrm{~s})$ contained little exploitable information (Figure 5g), the ANNA-

333 PALM reconstruction offered clear and detailed views of the disrupted microtubule 334 network (Figure 5h), exhibiting a much smaller number of filaments, with higher 335 curvature, than in untreated cells. These reconstructions were in good (though not 336 perfect) agreement with dense PALM images obtained from $K=60,000$ frames $337(K \Delta \mathrm{t}=10 \mathrm{~min})$ (Figure 5i). Thus, ANNA-PALM, when trained on microtubules in 
338 one experimental condition, may be successfully applied to new experimental 339 conditions without retraining, thereby highlighting the method's robustness to 340 biologically relevant structural alterations.

341 We further asked if ANNA-PALM is robust to changes in technical imaging 342 conditions. To address this, we performed localization microscopy on microtubules

343 by simultaneously changing multiple important imaging parameters relative to the

344 training data. Instead of PALM/STORM, we used DNA-PAINT a technique where 345 single molecule detection relies on transient binding of fluorophore-conjugated 346 short DNA strands to complementary, antibody-conjugated, DNA strands ${ }^{33}$, rather 347 than on fluorophore blinking. The continuously emitting freely diffusing dyes lead to 348 higher background noise in DNA-PAINT compared to PALM/STORM. Moreover, we 349 used primary mouse antibodies instead of rat antibodies, Cy3 dyes instead of Alexa350647 dyes, and an EMCCD instead of a sCMOS camera. Despite all these differences, 351 when ANNA-PALM was applied without retraining on a sparse microtubule image 352 ( $k=400$ frames, $\mathrm{k} \Delta \mathrm{t}=12 \mathrm{~s}$ ), the reconstructed image still agreed very well with the 353 dense DNA-PAINT image obtained from $K=60,000$ frames ( $K \Delta \mathrm{t}=30 \mathrm{~min}$ ) (Figure $5 \mathbf{j}$ 354 1). These data demonstrate the high robustness of ANNA-PALM to changes in 355 experimental imaging conditions.

357 ANNA-PALM adapts to different biological structures

358 To demonstrate that ANNA-PALM is not restricted to filamentary structure, we 359 turned to nuclear pores, a very different biological structure, and another popular 360 target of super-resolution imaging studies ${ }^{34-36}$. We retrained A-net simultaneously 
361 on microtubule images and on a single PALM image of the nucleoporin gp210 in

362 immunolabeled nuclear membranes of Xenopus frog eggs ${ }^{34,36}(K=30,000)$. With the

363 switch (Figure 1) set to microtubules ('MT'), this newly trained A-net can still

364 reconstruct sparse images of microtubules as when trained exclusively on

365 microtubule data (Supplementary Figure 13a-c). We then applied the same A-net

366 with the switch set to nuclear pores ('NPC') to reconstruct a new sparse PALM

367 image of gp210 obtained from the first $k=3,000$ frames (Figure 6a). The sparsity of

368 this image makes it difficult to clearly distinguish individual nuclear pores. ANNA-

369 PALM, however, reconstructs a much clearer image, containing many easily

370 identifiable ring-like structures, as expected for nuclear pores ${ }^{34}$ (Figure 6b), and in

371 good agreement with the dense PALM image obtained from $K=30,000$ frames (even

372 though the latter shows mostly incomplete, open rings, presumably due to

373 suboptimal labeling) (Figure 6c). An automated procedure based on cross-

374 correlation with a ring template indeed identified $\sim 2.7$ times more putative nuclear

375 pores from the ANNA-PALM image than the sparse image (Supplementary Figure

376 14a-c). Moreover, computed pore locations were in good agreement with a PALM

377 image of wheat germ agglutinin (WGA), a lectin that concentrates in the inner

378 nuclear pore channel ${ }^{36}$ (Supplementary Figure 14 d-f). These results show that

379 ANNA-PALM can successfully analyze non-filamentary structures, when properly

380 retrained, and that a single ANN, with a simple computational switch, can

381 reconstruct very different types of structures.

382 Finally, we imaged TOM22, a protein of the mitochondrial outer membrane ${ }^{37}$.

383 Whereas, at the resolution of our experiments, microtubules and nucleoporins are 
384 essentially one-dimensional and zero-dimensional structures, mitochondrial

385 membranes are two-dimensional surfaces. Furthermore, their complex 3D

386 morphology might seem less predictable than filaments or nuclear pores, potentially

387 hampering ANNA-PALM reconstruction. Despite these differences, after being

388 trained on nine PALM images of TOM22 (with frame numbers ranging from

$389 K=24,000$ to $K=40,000$ ), ANNA-PALM reconstructions of distinct sparse PALM

390 images ( $k=400$ frames) displayed mitochondrial morphologies in good agreement

391 with the dense PALM images (Figure 6d-f) - although the protein's localization

392 along the membrane was less well reproduced. Taken together, our results illustrate

393 the versatility of ANNA-PALM and its applicability to images of very different

394 structural content.

395

396 Discussion

397 We introduced ANNA-PALM, a computational method based on deep learning, that

398 reconstructs high quality super-resolution images from sparse, rapidly acquired, 399 single molecule localization data (and/or widefield images). Our method enables 400 considerable gains in acquisition time compared to standard localization 401 microscopy without increasing active fluorophore density, thereby preserving 402 spatial resolution. In fact, ANNA-PALM even improves spatial resolution when 403 applied to images of lower resolution than the training data 404 (Supplementary Figures 7a, 10), and greatly diminishes the detrimental effect of 405 drift. The improvement in imaging efficiency afforded by ANNA-PALM alleviates the 406 incompatibility between high resolution and high-throughput microscopy by 
407 enabling super-resolution imaging of thousands of cells within a few hours or even

408 less (Figure 4, Supplementary Figure 12). This will facilitate super-resolution

409 studies of rare events, cellular heterogeneity and of partly stochastic structures such

410 as cytoskeletal polymers or chromosomes, whose characterization requires

411 statistics on many configurations ${ }^{38,39}$. ANNA-PALM may also be beneficial for high-

412 throughput imaging screens, e.g. of drug treatments or gene knock-outs ${ }^{40-42}$. In

413 addition, we envision applications to super-resolution imaging of large samples by

414 stitching together multiple images of spatially adjacent fields. The ability to generate

415 images spanning many orders of magnitude in scale could be well adapted to

416 expansion microscopy, a super-resolution technique that physically increases

417 sample size, but often requires tiling many fields of view to image even a single

418 cell ${ }^{43,44}$. With correlative microscopy 45 , it might also be possible to train ANNA-

419 PALM to reconstruct electron microscopy (EM) images from fluorescence images,

420 potentially extending the method to molecular resolutions currently out of reach of

421 localization microscopy. Adaptation of ANNA-PALM to $3 \mathrm{D}^{15,46}$ and multi-color ${ }^{47,48}$

422 localization microscopy should be relatively straightforward. Localization

423 microscopy of cellular dynamics remains very challenging 3,49 . By using much fewer

424 frames (or even only widefield images), ANNA-PALM could dramatically improve

425 the temporal resolution of live cell localization microscopy without sacrificing

426 spatial resolution or increasing phototoxicity and photobleaching. Thus, ANNA-

427 PALM provides multiple novel avenues for multi-scale imaging beyond standard 428 spatio-temporal resolution limits. 
Neverthelesss, important caveats should be stressed. First, although ANNA-

430 PALM can be applied successfully to very different types of images (Figure 6), the

431 method fails in absence of statistical redundancies between molecular localizations,

432 e.g. for entirely random distributions of molecules. Second, ANNA-PALM requires

433 prior training on dense PALM images with structures similar to those in the images

434 to be reconstructed. We showed that ANNA-PALM is robust, i.e. does not require

435 retraining, for some experimentally induced changes in structures and variations in

436 imaging parameters (Figure 5). Nevertheless, indiscriminate application of ANNA-

437 PALM to very different structures without retraining, or incorrect setting of the

438 switch, may result in artifacts (Supplementary Figure 13h). Third, even when

439 applied to data similar to the training images, ANNA-PALM can produce errors -as

440 any reconstruction method in a context of information scarcity. The frequency of

441 errors can be reduced by increasing the number of recorded frames, at the cost of

442 reduced acceleration (see Figures 2,3 and Supplementary Figures 4, 8, 9). In

443 addition, ANNA-PALM can use widefield images to estimate the reliability of

444 reconstructions, thereby helping their interpretation, providing some protection

445 against artifacts and indicating when retraining may be needed (Supplementary

446 Figures 11, 15). Future work, e.g. using Bayesian deep learning50, may provide

447 additional assessments of reconstruction uncertainty and shed more light on the 448 scope and limitations of our approach.

449 As a purely computational technique, ANNA-PALM does not necessitate any

450 changes to existing microscopy systems, but only requires one or a few standard

451 PALM images for training. To facilitate its adoption and future development, we 
452 make our source code, an ImageJ plugin and a cloud-computing based web

453 application available on https://annapalm.pasteur.fr/ along with sample data.

454 Because the performance of deep learning methods improves with the amount and

455 variety of training data, we designed our web application to enable sharing of data

456 and trained ANNs. As ANNA-PALM will learn from an increasing large and diverse

457 collection of images, we expect it to reach even higher accuracy or efficiency and to

458 expand its scope of application in the future.

\section{Acknowledgements}

462 We thank the following colleagues for useful discussions and suggestions and/or

463 critical reading of the manuscript: C. Leduc, S. Etienne-Manneville, S. Lévêque-Fort,

464 N. Bourg, A. Echard, J-B. Masson, T. Rose, P. Hersen, F. Mueller, M. Cohen, Z. Zhang,

465 and P. Kanchanawong. We also thank the four anonymous reviewers for their

466 constructive criticism, which led to significant improvements of ANNA-PALM. We

467 further thank O. Faklaris, J. Sellés and M. Penrad (Institut Jacques Monod), and F.

468 Montel (Ecole Normale Supérieure de Lyon) for providing Xenopus nuclear pore

469 data, B. Jian (Institut Pasteur) for TOM22 antibodies, and C. Leterrier for fixation

470 protocols. We thank E. Rensen and C. Weber for help with experiments and

471 suggestions, B. Lelandais for help with PALM image processing, J-B. Arbona for

472 polymer simulations and J. Parmar for suggestions that led to the name ANNA-

473 PALM. We thank the IT service of Institut Pasteur, including J-B. Denis, N. Joly, and S.

474 Fournier, for access to the HPC cluster and relevant assistance, and T. Huynh for

475 help with GPU computing. This work was funded by Institut Pasteur, Agence 
476 Nationale de la Recherche grant (ANR 14 CE10 0018 02), Fondation pour la

477 Recherche Médicale (Equipe FRM, DEQ 20150331762), and the Région Ile de France 478 (DIM Malinf). We also acknowledge Investissement d'Avenir grant ANR-16-CONV4790005 for funding a GPU farm used in this work. A.A. and X.H. are recipients of 480 Pasteur-Roux fellowships from Institut Pasteur. W.O. is a scholar in the Pasteur 481 Paris University (PPU) International PhD program.

482

483

484 Author contributions

485 W.O.: conceived method, developed ANNA-PALM software and web application, 486 performed experiments and analyses. A.A., M.L., X.H.: performed experiments. C.Z.:

487 conceived method, supervised project and wrote manuscript. 488

490 Competing financial interests statement

491 W.O. and C.Z. are listed as inventors on European patent application EP17306022 492 filed by Institut Pasteur.

493

494 
495

496

497

498

499

500

501

502

503

504

505

506

507

508

509

510

511

512

513

514

515

516

517

518

519

520

521

522

523

524

525

526

527

528

529

530

531

532

533

534

535

536

537

538

539

540

\section{REFERENCES}

1. Betzig, E. et al. Imaging intracellular fluorescent proteins at nanometer resolution. Science (80-. ). 313, 1642-1645 (2006).

2. Rust, M. J., Bates, M. \& Zhuang, X. Sub-diffraction-limit imaging by stochastic optical reconstruction microscopy (STORM). Nat Methods 3, 793-795 (2006).

3. Legant, W. R. et al. High-density three-dimensional localization microscopy across large volumes. Nat. Methods 13, 359-365 (2016).

4. Deschout, H. et al. Precisely and accurately localizing single emitters in fluorescence microscopy. Nat. Methods 11, 253-266 (2014).

5. Jones, S. A., Shim, S. H., He, J. \& Zhuang, X. Fast, three-dimensional superresolution imaging of live cells. Nat Methods 8, 499-505 (2011).

6. Huang, F. et al. Video-rate nanoscopy using sCMOS camera-specific singlemolecule localization algorithms. Nat. Methods 10, 653-8 (2013).

7. Carlton, P. M. et al. Fast live simultaneous multiwavelength four-dimensional optical microscopy. Proc. Natl. Acad. Sci. U. S. A. 107, 16016-22 (2010).

8. Stelzer, E. H. K. Light-sheet fluorescence microscopy for quantitative biology. Nat. Methods 12, 23-26 (2014).

9. Huang, F., Schwartz, S. L., Byars, J. M. \& Lidke, K. A. Simultaneous multipleemitter fitting for single molecule super-resolution imaging. Biomed. Opt. Express 2, 1377-93 (2011).

10. Burnette, D. T., Sengupta, P., Dai, Y., Lippincott-Schwartz, J. \& Kachar, B. Bleaching/blinking assisted localization microscopy for superresolution imaging using standard fluorescent molecules. Proc Natl Acad Sci U S A 108, 21081-21086 (2011).

11. Simonson, P. D., Rothenberg, E. \& Selvin, P. R. Single-molecule-based superresolution images in the presence of multiple fluorophores. Nano Lett 11, 5090-5096 (2011).

12. Zhu, L., Zhang, W., Elnatan, D. \& Huang, B. Faster STORM using compressed sensing. Nat. Methods 9, 721-723 (2012).

13. Cox, S. et al. Bayesian localization microscopy reveals nanoscale podosome dynamics. Nat Methods 9, 195-200 (2012).

14. Ram, S., Ward, E. S. \& Ober, R. J. Beyond Rayleigh's criterion: A resolution measure with application to single-molecule microscopy. Proc. Natl. Acad. Sci. 103, 4457-4462 (2006).

15. Deschout, H. et al. Precisely and accurately localizing single emitters in fluorescence microscopy. Nat. Methods 11, 253-266 (2014).

16. LeCun, Y., Bengio, Y. \& Hinton, G. Deep learning. Nature 521, 436-444 (2015).

17. Michael, E. Sparse and Redundant Representations: From Theory to Applications in Signal and Image Processing. Springer (Springer Verlag, 2010).

18. Hinton, G. E. \& Salakhutdinov, R. R. Reducing the Dimensionality of Data with Neural Networks. Science (80-. ). 313, (2006).

19. Schmidhuber, J. Deep learning in neural networks: An overview. Neural Networks 61, 85-117 (2014).

20. Sage, D. et al. Quantitative evaluation of software packages for single-molecule localization microscopy. Nat. Methods 12, 717-724 (2015). 
541

542

543

544

545

546

547

548

549

550

551

552

553

554

555

556

557

558

559

560

561

562

563

564

565

566

567

568

569

570

571

572

573

574

575

576

577

578

579

580

581

582

583

584

585

586

21. Isola, P., Zhu, J.-Y., Zhou, T. \& Efros, A. A. Image-to-Image Translation with Conditional Adversarial Networks. http://arxiv.org/abs/1611.07004 (2016).

22. Ronneberger, O., Fischer, P. \& Brox, T. U-net: Convolutional networks for biomedical image segmentation. in Medical Image Computing and ComputerAssisted Intervention - MICCAI 2015. Lecture Notes in Computer Science, 9351, 234-241 (Springer International Publishing, 2015).

23. Goodfellow, I. et al. Generative Adversarial Nets. Adv. Neural Inf. Process. Syst. 27 2672-2680 (2014). doi:10.1017/CB09781139058452

24. Ciresan, D., Giusti Alessandro, Luca, G. \& Schmidhuber, J. Mitosis Detection in Breast Cancer Histology Images with Deep Neural Networks. Med. Image Comput. Comput. Interv. - MICCAI 2013 8150, (2013).

25. Wang, Z. W. Z. \& Bovik, A. C. A. C. Mean squared error: Love it or leave it? A new look at Signal Fidelity Measures. IEEE Signal Process. Mag. 26, 98-117 (2009).

26. Zhao, H., Gallo, O., Frosio, I. \& Kautz, J. Loss Functions for Image Restoration with Neural Networks. IEEE Trans. Comput. IMAGING 3, 47-57 (2017).

27. Culley, S. et al. NanoJ-SQUIRREL: quantitative mapping and minimisation of super-resolution optical imaging artefacts. bioRxiv 158279 (2017). doi:10.1101/158279

28. Srivastava, N., Hinton, G., Krizhevsky, A., Sutskever, I. \& Salakhutdinov, R. Dropout: A Simple Way to Prevent Neural Networks from Overfitting. J. Mach. Learn. Res. 15, (2014).

29. Arbona, J.-M., Herbert, S., Fabre, E. \& Zimmer, C. Inferring the physical properties of yeast chromatin through Bayesian analysis of whole nucleus simulations. Genome Biol. 18, 81 (2017).

30. Arnal, I. \& Wade, R. H. How does taxol stabilize microtubules? Curr. Biol. 5, 900-908 (1995).

31. $\mathrm{Wu}, \mathrm{S}$. et al. Microtubule motors regulate ISOC activation necessary to increase endothelial cell permeability. J. Biol. Chem. 282, 34801-8 (2007).

32. De Brabander, M., De May, J., Joniau, M. \& Geuens, G. Ultrastructural immunocytochemical distribution of tubulin in cultured cells treated with microtubule inhibitors. Cell Biol. Int. Rep. 1, 177-83 (1977).

33. Schnitzbauer, J., Strauss, M. T., Schlichthaerle, T., Schueder, F. \& Jungmann, R. Super-resolution microscopy with DNA-PAINT. Nat. Protoc. 12, 1198-1228 (2017).

34. Löschberger, A. et al. Super-resolution imaging visualizes the eightfold symmetry of gp210 proteins around the nuclear pore complex and resolves the central channel with nanometer resolution. J. Cell Sci. 125, 570-5 (2012).

35. Szymborska, A. et al. Nuclear pore scaffold structure analyzed by superresolution microscopy and particle averaging. Science 341, 655-8 (2013).

36. Sellés, J. et al. Nuclear pore complex plasticity during developmental process as revealed by super-resolution microscopy. Sci. Rep. 7, 14732 (2017).

37. Bellot, G. et al. TOM22, a core component of the mitochondria outer membrane protein translocation pore, is a mitochondrial receptor for the proapoptotic protein Bax. Cell Death Differ. 14, 785-794 (2007).

38. Boettiger, A. N. et al. Super-resolution imaging reveals distinct chromatin 
folding for different epigenetic states. Nature 529, 418-422 (2016).

39. Zhang, Z., Nishimura, Y. \& Kanchanawong, P. Extracting microtubule networks from superresolution single-molecule localization microscopy data. Mol. Biol. Cell 28, 333-345 (2017).

40. Neumann, B. et al. Phenotypic profiling of the human genome by time-lapse microscopy reveals cell division genes. Nature 464, 721-727 (2010).

41. Beghin, A. et al. Localization-based super-resolution imaging meets highcontent screening. Nat. Methods 14, 1184-1190 (2017).

42. Ouyang, W. \& Zimmer, C. The imaging tsunami: Computational opportunities and challenges. Curr. Opin. Syst. Biol. 4, 105-113 (2017).

43. Chen, F., Tillberg, P. W. \& Boyden, E. S. Expansion microscopy. Science (80-. ). 347, 543-548 (2015).

44. Chang, J.-B. et al. Iterative expansion microscopy. Nat. Methods (2017). doi:10.1038/nmeth.4261

45. de Boer, P., Hoogenboom, J. P. \& Giepmans, B. N. G. Correlated light and electron microscopy: ultrastructure lights up! Nat. Methods 12, 503-513 (2015).

46. Huang, B., Wang, W., Bates, M. \& Zhuang, X. Three-dimensional superresolution imaging by stochastic optical reconstruction microscopy. Science (80-. ). 319, 810-813 (2008).

47. Bates, M., Huang, B., Dempsey, G. T. \& Zhuang, X. Multicolor Super-Resolution Imaging with Photo-Switchable Fluorescent Probes. Science (80-. ). 1146598v1 (2007).

48. Jungmann, R. et al. Multiplexed 3D cellular super-resolution imaging with DNA-PAINT and Exchange-PAINT. Nat. Methods 11, 313-318 (2014).

49. Shroff, H., Galbraith, C. G., Galbraith, J. A. \& Betzig, E. Live-cell photoactivated localization microscopy of nanoscale adhesion dynamics. Nat Methods 5, 417423 (2008).

50. Kendall, A. \& Gal, Y. What Uncertainties Do We Need in Bayesian Deep Learning for Computer Vision? Adv. Neural Inf. Process. Syst. 30 (NIPS 2017) pre-proceedings 5580-5590 (2017). 
FIGURE LEGENDS

620

\section{FIGURE 1: Overview of ANNA-PALM}

622 ANNA-PALM consists of two main stages: (a) acquisition of training images using 623 standard localization microscopy (PALM) followed by artificial neural network 624 (ANN) training, and (b) reconstruction of super-resolution views and low resolution 625 error maps from new sparse PALM and/or widefield images (inference). 626 (a) Training images are obtained by acquiring one or a few long sequences, of $627 K \sim 10^{3}-10^{5}$ diffraction limited, single molecule image frames, as in standard PALM 628 experiments; optionally, a widefield image $\mathrm{W}$ can also be acquired (top). The 629 acquisition time for each image sequence is $K \Delta \mathrm{t}$, where $\Delta \mathrm{t}$ is the single frame 630 exposure time. Standard localization microscopy algorithms (grey 'localization' 631 boxes) are used to generate super-resolution images. For each experiment, a highly 632 sampled (dense) super-resolution image (PK) is generated using all (or in practice $633 \geq 95 \%$ ) available $K$ frames. Sparse PALM images (Pk) from the same experiment are 634 obtained by using only $k<<K$ frames. A switch (light blue) can be set to distinguish 635 different types of structures, e.g. nuclear pore complexes ('NPC'), mitochondria 636 ('Mito') or microtubules ('MT'). An ANN (labeled $\mathcal{G}$ for 'generator', orange) is trained 637 by using the sparse PALM images Pk (plus an upsampled version $\mathrm{L}$ of the widefield 638 image $\mathrm{W}$ and the switch setting) as inputs and the corresponding dense PALM image 639 PK as target output. During training, the output of the generator $\mathcal{G}$ (Ak) is compared 640 to the target image PK and the widefield image L (if available) via three loss, or error

641 functions (gray bordered boxes): (i) the 'super-resolution reconstruction error' 
642 measures the difference between the reconstructed image Ak and the target PK 643 using a combination of the L1 norm and the MS-SSIM; (ii) the "low resolution

644 reconstruction error' measures the MS-SSIM between the low resolution image WA 645 predicted from the reconstruction Ak and the low resolution image WP predicted 646 from the target image PK. Images WA and WP are predicted using a second ANN, 647 called low-resolution estimator (labeled $Q$, blue) that is trained to produce an 648 approximation of the actual widefield image W based on the MS-SSIM metric; (iii) 649 the 'conditional GAN error' uses a third ANN (labeled $D$ for 'discriminator', red) that 650 attempts to distinguish between real dense PALM images PK and the generator's 651 output Ak. The combined loss functions are iteratively optimized using stochastic

652 gradient descent. (b) A short sequence of diffraction limited single molecule images 653 (with $k<<K$ frames, i.e. acquisition time $k \Delta \mathrm{t}$ ), and an optional widefield image ( $\mathrm{W}^{\prime}$ ), 654 are acquired. Standard localization algorithms generate a sparse (under-sampled) 655 PALM image ( $\mathrm{P}^{\prime} \mathrm{k}$ ). This sparse image P'k (and the upscaled widefield image L' and 656 switch setting) are fed as inputs to the trained generator $\mathcal{G}$, which outputs a 657 reconstructed ANNA-PALM image ( $\left.\mathrm{A}^{\prime} \mathrm{k}\right)$. In addition, the low resolution estimator $Q$ 658 predicts a low resolution image WA', which can be compared to the input widefield 659 image W' via the MS-SSIM to produce a low resolution error map (top). 660

\section{FIGURE 2: Validation of ANNA-PALM on simulated images}

662 (a) Simulated widefield image of microtubules. (b) Simulated sparse PALM image of 663 microtubules with $n=6,834$ localizations. (c) ANNA-PALM reconstruction using only 664 the widefield image a as input. (d) ANNA-PALM reconstruction using both the 
665 widefield image a and the sparse PALM $\mathbf{b}$ image as inputs. (e) Simulated "perfect" 666 PALM image, equivalent to a PALM image with an infinite number of localizations $667(n=\infty)$ and a resolution of $23 \mathrm{~nm}$. This image was used to generate a (by convolution 668 with a Gaussian kernel approximating the microscope point spread function) and $\mathbf{b}$ 669 (by application of Poisson noise). (f) Merged image showing the perfect PALM image $670 \mathrm{e}$ in green and the ANNA-PALM reconstruction $\mathbf{d}$ in red. Note that the ANNA-PALM 671 images c,d provide many high resolution details that are absent from the widefield 672 image a and the sparse PALM image $\mathbf{b}$ and that are in good (c) or very good (d) 673 agreement with the perfect PALM image e. Some reconstruction errors are 674 highlighted by arrows. Blue arrows in panel c point to errors of ANNA-PALM 675 reconstruction from the widefield image only, the white arrow in panel $\mathbf{d}$ points to 676 an error of ANNA-PALM reconstruction from both widefield and sparse PALM 677 images combined. Reconstruction errors diminish for larger numbers of 678 localizations, $n$ (Supplementary Figure 4). (g) Reconstruction quality of PALM and 679 ANNA-PALM images, measured by the MS-SSIM with the perfect PALM image e, as 680 function of localization number $n$. Black curve: reconstruction quality of the 681 standard PALM images. Dashed blue curve: reconstruction quality of ANNA-PALM 682 using the sparse PALM images as input. Solid blue curve: reconstruction quality of 683 ANNA-PALM using both the sparse PALM and widefield images as inputs. Red 684 dashed line: reconstruction quality of ANNA-PALM using the widefield images as 685 input only. Dots are averages from 10 simulations; error bars show standard 686 deviations. The vertical dashed orange line indicates the minimum number of 687 localizations needed to achieve a resolution of $R_{5 \times \mathrm{Nyq}}=23 \mathrm{~nm}$ according to the five- 
688 fold Nyquist criterion ${ }^{3}$. The dashed grey line indicates the minimum number of 689 localizations needed to achieve a double mean nearest neighbor distance less than $69023 \mathrm{~nm}$. ANNA-PALM reconstructions from sparse PALM images only (i.e. without 691 widefield images) achieve the same average MS-SSIM as standard PALM at the five692 fold Nyquist sampling limit with 26 times less localizations (blue double arrow). 693 ANNA-PALM reconstruction quality is highest when using both widefield and sparse 694 PALM images as inputs.

695

\section{FIGURE 3: ANNA-PALM imaging of microtubules}

697 ANNA-PALM reconstructions of a localization microscopy image of immunostained 698 microtubules. (a) Widefield image. (b) Sparse PALM image obtained from the first $6999 \mathrm{~s}$ of acquisition ( $k=300$ frames, $n=11,740$ localizations). (c) Dense PALM image 700 obtained from a 15 min long acquisition $(K=30,000$ frames, $N=409,364$

701 localizations). (d) ANNA-PALM reconstruction from the widefield image a only.

702 (e) ANNA-PALM reconstruction from the sparse PALM image b only. (f) ANNA-

703 PALM reconstruction from the widefield image a and sparse PLAM image b

704 combined. In panels b-f, pixel values are linearly mapped to colors from the look-up 705 table shown below. Black and white correspond to values $V_{\min }$ and $V_{\max }$, 706 respectively, with $V_{\min }=0$ for all panels, $V_{\max }=3,24,102,102$ and 102 for panels $\mathbf{b}, \mathbf{c}$, 707 d, e and f, respectively. (g-i) Merged images comparing ANNA-PALM 708 reconstructions from panels d-f to the dense PALM image c. ANNA-PALM 709 reconstructions are shown in red, the dense PALM image in green. (j) Gradual 710 improvement of image quality for increasing acquisition time $k \Delta t$, shown for the 
711 area highlighted in the insets of panels a-i. Top row: sparse PALM images. Middle

712 row: ANNA-PALM reconstructions from the sparse PALM images only (without

713 widefield). Bottom row: ANNA-PALM reconstructions from the widefield and sparse

714 PALM images combined. Supplementary Video 1 shows the gradual increase in

715 quality of PALM and ANNA-PALM images with increased acquisition time for the

716 larger region of interest shown in panels a-i.

717

718 FIGURE 4: High-throughput imaging with ANNA-PALM

719 Application of ANNA-PALM to high-throughput imaging of a $1.8 \mathrm{~mm} \times 1.8 \mathrm{~mm}$ area

720 containing more than 1,000 cells. (a) Sparse PALM image of this area, constructed

721 by assembling a mosaic of 33×33=1,089 sparse PALM images of individual fields of

722 view, obtained from $k=1,000$ raw frames each (with $\Delta \mathrm{t}=10 \mathrm{~ms}$ exposure time per

723 frame, i.e. in $k \Delta t=10 \mathrm{~s}$ ). Total image acquisition time was $1,089 \times 10$ s, i.e. $\sim 3.1$ hours.

724 The sparsity of the image is not apparent at this large scale. (b) ANNA-PALM

725 reconstruction of the image in a, obtained by assembling a mosaic of 1,089

726 individual reconstructions (one per field of view). (c) Magnified view of the green

727 boxed region in a. The inset shows a further magnified view of the yellow boxed

728 region, highlighting the sparsity of the image. (d) Same as c, but for the ANNA-PALM

729 reconstruction. A line profile across a microtubule is shown, with a FWHM $\approx 51 \mathrm{~nm}$.

730 Non-linear contrast adjustment was applied manually for panels $\mathbf{a}$ and $\mathbf{b}$, with black

731 corresponding to values of zero in both panels. In panels $\mathbf{c}$ and $\mathbf{d}$, pixel values were

732 linearly mapped to colors from the look-up table in Figure 3; Black and white

733 correspond to values $V_{\min }$ and $V_{\max }$, respectively, with $V_{\min }=0$ for all panels, and 
$734 \mathrm{~V}_{\max }=3$ and 51 for panels $\mathbf{c}$ and $\mathbf{d}$, respectively. See also Supplementary Video 2 for

735 an animated 'zoom-in' highlighting the spatial scales covered by the assembled

736 image. See also Supplementary Figure 12 for ANNA-PALM reconstructions of the

737 same area from the widefield images only.

738

739 FIGURE 5: Robustness of ANNA-PALM to experimental perturbations

740 This figure shows ANNA-PALM reconstructions using an ANN trained on PALM

741 images of microtubules in untreated cells and applied without retraining to sparse

742 localization images of microtubules in different experimental conditions: untreated

743 control cells (a-c); cells treated with $1 \mu \mathrm{M}$ of Taxol (d-f); cells treated with $1 \mu \mathrm{M}$ of

744 Nocodazole (g-i); untreated cells imaged with DNA-PAINT (j-1). (a,d,g,j) Sparse

745 localization images obtained from the first $k$ frames of the acquired image sequence,

746 with $k=500,800,300$, and 400 for $\mathbf{a}, \mathbf{d}, \mathbf{g}$, and $\mathbf{j}$, respectively. (b,e,h,k) ANNA-PALM

747 reconstructions using the sparse localization images immediately to the left as

748 input. (c,f,i,l) Dense localization images obtained from $K=60,000$ frames. Pixel

749 values are linearly mapped to colors from the look-up table in Figure 3. Black and

750 white correspond to values $V_{\min }$ and $V_{\max }$, respectively, with $V_{\min }=0$ for all panels,

751 and $V_{\max }=10,120,90,25,150,40,18,150,50,18,120$, and 200 for panels a, b, c, d,

$752 \mathbf{e}, \mathbf{f}, \mathbf{g}, \mathbf{h}, \mathbf{i}, \mathbf{j}, \mathbf{k}$, and $\mathbf{l}$, respectively.

753

754 FIGURE 6: ANNA-PALM reconstructions of nuclear pores and mitochondria

755 PALM and ANNA-PALM images of nuclear pores (a-c) and mitochondria (d-f).

756 (a) Sparse PALM image of the immunolabeled Xenopus nucleoporin gp210 obtained 
757 from the first $k=3,000$ frames. Note that individual nuclear pores are hard to 758 identify. (b) ANNA-PALM reconstruction of image a. (c) Dense PALM image obtained 759 from all $K=30,000$ frames. (d) Sparse PALM image of the immunolabeled 760 mitochondrial outer membrane protein TOM22, obtained from the first $k=400$ 761 frames. (e) ANNA-PALM reconstruction of image d. (f) Dense PALM image obtained 762 from all $K=30,000$ frames. Pixel values are linearly mapped to colors from the look763 up table shown in Figure 3. Black and white correspond to values $V_{\min }$ and $V_{\max }$ 764 respectively, with $V_{\min }=0$ for all panels, and $V_{\max }=3,51,3,3,128$, and 18 for panels a, 765 b, c, d, e, and f, respectively 


\section{Artificial neural network}

769 Our ANN, called 'A-net', is based on the pix2pix architecture ${ }^{21}$, which is a special

770 conditional generative adversarial network (cGAN) ${ }^{23}$ for image to image

771 "translation", i.e. mapping from one type of image to another. The A-net consists of

772 three distinct neural networks: (i) a generator network $\mathcal{G}$ that produces the

773 reconstructed super-resolution image, (ii) a network $Q$ called 'low resolution

774 estimator' that produces the low resolution error map, (iii) a cGAN discriminator

775 network $D$ that provides the adversarial loss (Figure 1a). The generator network $\mathcal{G}$

776 builds on the U-net architecture, and consists of an encoder-decoder network with

777 skip connections ${ }^{22}$ and 16 convolutional layers. Its inputs and outputs are image

778 patches containing $(256 \mathrm{~m}) \mathrm{x}(256 \mathrm{~m})$ pixels, where $m$ is an integer (we used $m=1$ or 2

779 but this can be adjusted for different sizes of CPU/GPU memory or input images).

780 The input is a sparse PALM image, a widefield image upscaled to the same size (see

781 below), and a computational switch number that allows the network to switch

782 between different types of image structures (e.g. nuclear pores or microtubules).

783 The switch setting is encoded numerically and coupled by convolutional operations

784 into the A-net encoder. The output of the generator $\mathcal{G}$ is a reconstructed image

785 (called ANNA-PALM reconstruction or ANNA-PALM image elsewhere) of the same

786 size as the input images. The low resolution estimator $Q$ has four convolutional

787 layers. It takes the $(256 \mathrm{~m}) \times(256 \mathrm{~m})$ dense PALM image patch or the ANNA-PALM

788 image patch as input and outputs a low resolution image with $(64 \mathrm{~m}) \mathrm{x}(64 \mathrm{~m})$ pixels. 
789 The cGAN discriminator network $D$ has five convolutional layers. Its inputs are three $790(256 \mathrm{~m}) \mathrm{x}(256 \mathrm{~m})$ pixel image patches (the sparse PALM image, the widefield image, 791 and either the ANNA-PALM reconstruction or the corresponding dense PALM 792 image), plus the upscaled widefield image, and its output is a $(30 \mathrm{~m}) \mathrm{x}(30 \mathrm{~m})$ image 793 whose pixel values indicate whether the corresponding input patch is real (i.e. an 794 experimentally obtained conventional PALM image) or produced by the generator $\mathcal{G}$.

795 All convolutional layers are followed by batch normalization ${ }^{51}$. Dropout layers ${ }^{28}$ 796 (with dropout probability $\mathrm{p}=0.5$ ) are introduced in the central layers of the A-net 797 generator and turned on during training, but switched off during inference. 798 Activation functions are rectified linear units (ReLU) $x \rightarrow \sup (x, 0)$ or "leaky" ReLUs $799 x \rightarrow \sup (x, 0)+\inf (\varepsilon x, 0)$ with $\varepsilon=0.2^{52}$, except for the last layer of $\mathcal{G}$, which uses the 800 hyperbolic tangent $x \rightarrow \tanh (x)$ and the last layer of $Q$, which uses a sigmoid 801 function $x \rightarrow(1+\exp (-x))^{-1}$. The A-net architecture is fully described in 802 Supplementary Note 1 and Supplementary Tables 1-4.

804 Training objectives and error map

805 Each of the three networks mentioned above $(\mathcal{G}, Q$, and $D)$ is associated to a distinct 806 objective function - also called loss- and hereafter noted $\mathscr{L}_{\mathcal{G}}, \mathscr{L}_{Q}$, and $\mathscr{L}_{D}$ respectively. 807 These loss functions are specified in detail below. In the following equations, for 808 notational simplicity, we designate the sparse input image as $\boldsymbol{S}$, the low resolution 809 (widefield) input image as $\boldsymbol{W}$, the corresponding dense PALM image (i.e. the target) 810 as $\boldsymbol{T}$, and the A-net's output as $\boldsymbol{A}=\mathcal{G}(\boldsymbol{S}, \boldsymbol{W})$ (in Figure 1a, images $\boldsymbol{S}, \boldsymbol{T}$ and $\boldsymbol{A}$ are 811 labeled Pk, PK and Ak, respectively); the computational switch indicating the image 
812 type is noted $\boldsymbol{M}$. Low resolution images produced by the low resolution estimator 813 network $Q$ from $\boldsymbol{A}$ and $\boldsymbol{T}$ are designated as $\boldsymbol{W}_{A}=Q(\boldsymbol{A})$ and $\boldsymbol{W}_{T}=Q(\boldsymbol{T})$, 814 respectively.

815 The generator loss function $\mathscr{L}_{\mathcal{G}}$ is the sum of three terms. The first term of $\mathscr{L}_{\mathcal{G}}$ 816 is the super-resolution reconstruction error, hereafter called $\mathscr{L}_{\text {SuperRes }}(\mathcal{G})$. This term 817 penalizes the difference between the generator output $\boldsymbol{A}$ and the target image $\boldsymbol{T}$. 818 Based on a recent analysis of loss functions for image restoration with neural 819 networks ${ }^{26}$, we defined this difference as a weighted average of two quantities: 820 (i) the multi-scale structural similarity index (MS-SSIM) between $\boldsymbol{A}$ and $\boldsymbol{T}$ and (ii) a 821 modification of the L1 norm, where the absolute difference between $\boldsymbol{A}$ and $\boldsymbol{T}$ is 822 smoothed by a Gaussian kernel:

$$
\begin{aligned}
& \mathscr{L}_{\text {SuperRes }}(\mathcal{G})=\mathbb{E}_{(\boldsymbol{S}, \boldsymbol{T}, \boldsymbol{W}, \boldsymbol{M}) \sim p_{\text {data }}(\boldsymbol{S}, \boldsymbol{T}, \boldsymbol{W}, \boldsymbol{M})}\left[\rho\left(1-\operatorname{MS} \_\operatorname{SSIM}(\boldsymbol{A}, \boldsymbol{T})\right)+(1\right. \\
& \left.\quad-\rho)\left\langle G_{\sigma} *|\boldsymbol{A}-\boldsymbol{T}|\right\rangle\right] \\
& \quad=\mathbb{E}_{(\boldsymbol{S}, \boldsymbol{T}, \boldsymbol{W}, \boldsymbol{M}) \sim p_{\text {data }}(\boldsymbol{S}, \boldsymbol{T}, \boldsymbol{W}, \boldsymbol{M})}\left[\rho\left(1-\operatorname{MS} \operatorname{SSIM}_{(\mathcal{G}}(\boldsymbol{S}, \boldsymbol{W}, \boldsymbol{M}), \boldsymbol{T}\right)\right)+(1 \\
& \left.\quad-\rho)\left\langle G_{\sigma} *|\mathcal{G}(\boldsymbol{S}, \boldsymbol{W}, \boldsymbol{M})-\boldsymbol{T}|\right\rangle\right]
\end{aligned}
$$

823 where $\mathbb{E}$ denotes expectation; $p_{\text {data }}(\boldsymbol{S}, \boldsymbol{T}, \boldsymbol{W}, \boldsymbol{M})$ is the joint probability density of the 824 sparse PALM images $\boldsymbol{S}$, dense PALM images $\boldsymbol{T}$, widefield images $\boldsymbol{W}$ and switch 825 settings $\boldsymbol{M}$ from the training data set; $\operatorname{MS} \operatorname{SSIM}(\boldsymbol{A}, \boldsymbol{T})$ is the multi-scale structural 826 similarity index between $\boldsymbol{A}$ and $\boldsymbol{T} ; G_{\sigma}$ is a Gaussian smoothing kernel; * denotes 827 convolution; $|\boldsymbol{A}-\boldsymbol{T}|$ is the absolute difference image (i.e. pixel (i,j) has value $828|\boldsymbol{A}(\mathrm{i}, \mathrm{j})-\boldsymbol{T}(\mathrm{i}, \mathrm{j})|$ and $\rho \in[0,1]$ is a scalar weight that balances the relative 829 contributions of MS-SSIM and the modified L1 norm and is set to $\rho=0.84$ as in ref. 83026. 
832 between the low resolution images $\boldsymbol{W}_{A}$ and $\boldsymbol{W}_{T}$ predicted by the low resolution 833 estimator network $Q$ :

$$
\begin{aligned}
\mathscr{L}_{\text {LowRes }}(\mathcal{G}, Q) & =\mathbb{E}_{(\boldsymbol{S}, \boldsymbol{T}, \boldsymbol{W}, \boldsymbol{M}) \sim p_{\mathrm{data}}(\boldsymbol{S}, \boldsymbol{T}, \boldsymbol{W}, \boldsymbol{M})}\left[1-\operatorname{MS} \operatorname{SSIM}\left(\boldsymbol{W}_{A}, \boldsymbol{W}_{T}\right)\right] \\
& =\mathbb{E}_{(\boldsymbol{S}, \boldsymbol{T}, \boldsymbol{W}, \boldsymbol{M}) \sim p_{\text {data }}(\boldsymbol{S}, \boldsymbol{T}, \boldsymbol{W}, \boldsymbol{M})}[1-\operatorname{MS} \operatorname{SSIM}(Q(\mathcal{G}(\boldsymbol{S}, \boldsymbol{W}, \boldsymbol{M})), Q(\boldsymbol{T}))]
\end{aligned}
$$

834 Alternatively, in the above objective function, $\boldsymbol{W}_{T}$ can be replaced by the actually 835 observed widefield image $\boldsymbol{W}$, although with our data this led to slightly lower 836 reconstruction quality. The low resolution estimator network $Q$ is trained 837 simultaneously with the generator $\mathcal{G}$ to produce a low resolution image from the 838 dense PALM image $\boldsymbol{T}$ that is consistent with the observed low resolution image $\boldsymbol{W}$.

839 This training is done based on the following objective function:

$$
\mathscr{L}_{Q}(Q)=\mathbb{E}_{(\boldsymbol{T}, \boldsymbol{W}) \sim p_{\text {data }}(\boldsymbol{T}, \boldsymbol{W})}\left[1-M S_{-} \operatorname{SSIM}(Q(\boldsymbol{T}), \boldsymbol{W})\right]
$$

840 Note that the reconstructed low resolution image $Q(T)$ is four times smaller than

841 the dense PALM image $\boldsymbol{T}$, as described in Supplementary Note 1. Because the input 842 widefield image $\boldsymbol{W}$ can have a different size, we use bilinear interpolation to resize $843 \boldsymbol{W}$ to the same size as $Q(\boldsymbol{T})$. (If needed, a scaling factor different from four can be 844 obtained by adding or removing downsample layers in network $Q$ ). At inference, the 845 low resolution estimator $Q$ is also used to produce the error map, as shown in 846 Figure 1b, and Supplementary Figures 6, 11, 15. This error map is defined as:

$$
\boldsymbol{E}_{Q}(\boldsymbol{A}, \boldsymbol{W})=\left(1-M S_{-} \operatorname{SSIM}(Q(\boldsymbol{A}), \boldsymbol{W})\right)(Q(\boldsymbol{A})+\boldsymbol{W})
$$

847 High (respectively low) values of the error map indicate large (respectively small) 848 inconsistencies, between the reconstructed super-resolution image $\boldsymbol{A}$ and the 849 observed widefield image $\boldsymbol{W}$. 
851 networks (GAN) $)^{21,23,53}$ and is noted $\mathscr{L}_{\mathrm{CGAN}}(\mathcal{G}, \mathcal{D})$. In a GAN, a generator network $\mathcal{G}$

852 learns to transform random input vectors $\mathbf{z}$ (drawn from a probability density $853 p_{\mathrm{z}}(\mathbf{z})$ ) into new samples of a data probability density $p_{\text {data }}(\boldsymbol{x})$. In our case, the data 854 samples $\boldsymbol{x}$ are the dense PALM images $\boldsymbol{T}$. The generator $\mathcal{G}$ learns by working against 855 a discriminator network $\mathcal{D}$ that simultaneously learns to discriminate between 856 original data samples and samples generated by $\mathcal{G}$. Adversarial training thus 857 consists in playing a minmax game such that $\left(\mathcal{G}^{*}, \mathcal{D}^{*}\right)=\arg \min _{\mathcal{G}} \max _{\mathcal{D}} \mathcal{L}_{\mathrm{GAN}}(\mathcal{G}, \mathcal{D})$, 858 with an objective function of the form ${ }^{23}: \mathcal{L}_{\mathrm{GAN}}(\mathcal{G}, \mathcal{D})=\mathbb{E}_{\boldsymbol{x} \sim p_{\text {data }}(\boldsymbol{x})}[\log \mathcal{D}(\boldsymbol{x})]+$ $859 \mathbb{E}_{\mathbf{z} \sim p_{\mathbf{Z}}(\mathbf{z})} \log [1-\mathcal{D}(\mathcal{G}(\mathbf{z}))]$, or equivalently by simultaneous optimization of two 860 coupled loss functions:

$$
\left\{\begin{array}{c}
\mathcal{D}^{*}=\arg \max _{\mathcal{D}}\left(\mathbb{E}_{\boldsymbol{x} \sim p_{\text {data }}(\boldsymbol{x})}[\log \mathcal{D}(\boldsymbol{x})]+\mathbb{E}_{\mathbf{z} \sim p_{\mathbf{Z}}(\mathbf{z})} \log [1-\mathcal{D}(\mathcal{G}(\mathbf{z}))]\right) \\
\mathcal{G}^{*}=\arg \min _{G}\left(\mathbb{E}_{\mathbf{z} \sim p_{\mathbf{Z}}(\mathbf{z})} \log [1-\mathcal{D}(\mathcal{G}(\mathbf{z}))]\right)
\end{array}\right.
$$

861 In a conditional GAN ( $\mathrm{CGAN}$ ), the generator and the discriminator have an extra 862 input vector $\boldsymbol{c}$ and the first objective function above becomes: $\mathcal{L}_{\mathcal{D}}(\mathcal{G}, \mathcal{D})=$ $863 \mathbb{E}_{(\boldsymbol{c}, \boldsymbol{x}) \sim p_{\text {data }}(\boldsymbol{c}, \boldsymbol{x})}[\log \mathcal{D}(\boldsymbol{c}, \boldsymbol{x})]+\mathbb{E}_{\boldsymbol{c} \sim p_{\text {data }}(\boldsymbol{c}), \mathbf{z} \sim p_{\mathrm{Z}}(\mathbf{z})} \log [1-\mathcal{D}(\boldsymbol{c}, \mathcal{G}(\boldsymbol{c}, \boldsymbol{z}))]$, such that the 864 generator learns a conditional probability density $p_{\text {data }}(\boldsymbol{x} \mid \boldsymbol{c})$; and the second 865 objective function likewise becomes $\mathcal{L}_{\mathrm{cGAN}}(\mathcal{G}, \mathcal{D})=\mathbb{E}_{\boldsymbol{c} \sim p_{\text {data }}(c), \boldsymbol{z} \sim p_{\mathrm{z}}(\mathbf{z})} \log [1-$ $866 \mathcal{D}(\mathcal{G}(\mathbf{z}))]$. In our A-net, we replaced the logarithmic losses above by least square $867 \operatorname{losses}^{53}$, as they empirically yielded better results. Thus, we used the objective 868 functions:

$$
\mathcal{L}_{\mathcal{D}}(\mathcal{G}, \mathcal{D})=\mathbb{E}_{(\boldsymbol{c}, \boldsymbol{x}) \sim p_{\text {data }}(\boldsymbol{c}, \boldsymbol{x})}(\mathcal{D}(\boldsymbol{c}, \boldsymbol{x})-1)^{2}+\mathbb{E}_{\boldsymbol{c} \sim p_{\text {data }}(\boldsymbol{c}), \mathbf{z} \sim p_{\mathrm{z}}(\mathbf{z})}[\mathcal{D}(\boldsymbol{c}, \mathcal{G}(\boldsymbol{c}, \boldsymbol{z}))]^{2} \quad \text { and }
$$


$870 \mathcal{L}_{\mathrm{cGAN}}(\mathcal{G}, \mathcal{D})=\mathbb{E}_{\boldsymbol{c} \sim p_{\text {data }}(\boldsymbol{c}), \boldsymbol{z} \sim p_{\mathrm{Z}}(\mathbf{z})}[1-\mathcal{D}(\boldsymbol{c}, \mathcal{G}(\boldsymbol{c}, \boldsymbol{z}))]^{2}$. In our case, the input $\boldsymbol{c}$ is the 871 sparse PALM image $\boldsymbol{S}$ combined with the upsampled version $\boldsymbol{L}=\mathcal{B}(\boldsymbol{W})$ of the 872 widefield image $\boldsymbol{W}$, where $\mathcal{B}$ denotes bilinear interpolation. Note that in practice the 873 noise $\mathbf{z}$ in our ANN was introduced only through the use of dropout layers, as in the 874 pix2pix implementation ${ }^{21}$. Thus, the objective functions are:

$$
\begin{aligned}
\mathscr{L}_{\mathcal{D}}(\mathcal{D})=\mathbb{E}_{\boldsymbol{S}, \boldsymbol{T}, \boldsymbol{W}, \boldsymbol{M} \sim p_{\text {data }}(\boldsymbol{S}, \boldsymbol{T}, \boldsymbol{W}, \boldsymbol{M})}(\mathcal{D}(\mathcal{B}(\boldsymbol{W}), \boldsymbol{S}, \boldsymbol{T})-1)^{2} \\
\quad+\mathbb{E}_{\boldsymbol{Z} \sim p_{\mathrm{Z}}(\boldsymbol{z}), \boldsymbol{S} \sim p_{\text {data }}(\boldsymbol{S})}(\mathcal{D}(\mathcal{B}(\boldsymbol{W}), \boldsymbol{S}, \mathcal{G}(\boldsymbol{S}, \boldsymbol{W}, \boldsymbol{M})))^{2}
\end{aligned}
$$

875 and:

$$
\mathscr{L}_{\mathrm{CGAN}}(\mathcal{G}, \mathcal{D})=\mathbb{E}_{(\boldsymbol{S}, \boldsymbol{T}, \boldsymbol{W}, \boldsymbol{M}) \sim p_{\mathrm{data}}(S, T, W, M)}(\mathcal{D}(\mathcal{B}(\boldsymbol{W}), \boldsymbol{S}, \mathcal{G}(\boldsymbol{S}, \boldsymbol{W}, \boldsymbol{M}))-1)^{2}
$$

876

In the end, combining the three loss terms described above, we implemented 877 the following optimization problem :

$$
\left\{\begin{array}{c}
Q^{*}=\arg \min _{Q} \mathscr{L}_{Q}(Q) \\
\mathcal{D}^{*}=\arg \max _{\mathcal{D}} \mathscr{L}_{\mathcal{D}}(\mathcal{D}) \\
\mathcal{G}^{*}=\arg \min _{\mathcal{G}}\left[\alpha \mathscr{L}_{\text {SuperRes }}(\mathcal{G})+\beta \mathscr{L}_{\text {LowRes }}(\mathcal{G}, Q)+\gamma \mathscr{L}_{\text {CGAN }}(\mathcal{G}, \mathcal{D})\right]
\end{array}\right.
$$

878 The weights $\alpha, \beta$ and $\gamma$ are hyperparameters, which we set manually to $\alpha=50$, $879 \beta=25$ and $\gamma=1$ for most experiments. In absence of widefield images $\boldsymbol{W}, \beta$ was 880 simply set to zero. The reported results are not very sensitive to these parameters.

881 We trained the A-net end-to-end using stochastic gradient descent (SGD) 882 with Adam $^{54}$ and a batch size of 1 with 200,000 or more iterations (backpropagation 883 steps). Our implementation was adapted from affinelayer's TensorFlow 55 884 implementation, which is ported from the Torch implementation of pix2pix ${ }^{21}$. Both 885 network training and inference were performed on Tesla P100, Tesla M40, Tesla 
886 K80 or GTX TitanXP graphical processing units (GPUs) from Nvidia. A-net training 887 from scratch typically takes from hours to days on a single GPU. Once trained, the A888 net takes only $\sim 1$ second or less to reconstruct a super-resolution image of $8892560 \times 2560$ pixels (corresponding to an entire FoV). Training time could be further 890 reduced by pretraining (or transfer learning), use of GPU clusters, or optimized data 891 augmentation.

892

893 Experimental training images and data augmentation

894 Experimental training data are obtained from standard localization microscopy data 895 (dense PALM images). To achieve good performance, ANNs generally necessitate 896 large amounts of training data. However, ANNA-PALM typically requires PALM 897 images from no more than 10 FoVs (of $55 \mu \mathrm{m} \times 55 \mu \mathrm{m}$ each) and can even be trained 898 with a single FoV. This is possible thanks to an extensive on-the-fly data 899 augmentation strategy, as described below. Each of the dense PALM training images 900 corresponds to a list of localizations $\left(f_{i}, x_{i}, y_{i}\right)_{i=1 . . n}$, where $f_{i} \in[1, K]$ is the index of 901 the diffraction limited frame from which localization $x_{i}, y_{i}$ originates, and $K$ is the 902 total frame number. PALM images are obtained as plain 2D histograms of these 903 localizations with typical pixel sizes of $10-20 \mathrm{~nm}$. From each list of localizations 904 (corresponding to a dense PALM image of a single FoV), we generate 10-30 pairs of 905 input and target images $(\boldsymbol{S}, \boldsymbol{T})$ for training. To define the target image $\boldsymbol{T}$, we take a 906 random consecutive subset $\left[k_{T}, k_{T}+0.95 K\right]$ of $95 \%$ of all available $K$ frames $\left(k_{T}\right.$ is 907 chosen randomly between 0 and $0.05 K$ ) and create the 2D histogram image based 908 on localizations from those frames only, i.e. from all $\left(x_{j}, y_{j}\right)$ such that $f_{j} \in$ 
$909\left[k_{T}, k_{T}+0.95 K\right]$. To define the sparse input image $\boldsymbol{S}$, we take random subsets of

$910300-500$ consecutive frames from the first half of the image sequence and similarly

911 create a 2D histogram of the localizations from those frames only. When a widefield

912 image $\boldsymbol{W}$ is available, this image must first be aligned with the corresponding dense

913 PALM image $\boldsymbol{T}$. This is done using an FFT-based phase correlation algorithm ${ }^{56}$ after

914 histogram equalization of image $\boldsymbol{T}$, smoothing by convolution with a Gaussian

915 Kernel of standard deviation 6 pixels, and resizing with bilinear interpolation to the

916 same size as image $\boldsymbol{W}$. The registered widefield image $\boldsymbol{W}$ is then scaled up using

917 bilinear interpolation to an image $\boldsymbol{L}=\mathcal{B}(\boldsymbol{W})$ with the same size as image $\boldsymbol{T}$.

918 During training, for each iteration of SGD, we crop the images $\boldsymbol{S}, \boldsymbol{L}$ and $\boldsymbol{T}$ with

919 a randomly placed $712 \times 712$ pixel sized region $\mathscr{R}=\left[x_{\min }, x_{\min }+712\right] \times$

$920\left[y_{\min }, y_{\min }+712\right]$. We then use random geometric transformations and apply them

921 identically to the three images. Specifically, we rotate the images by a random angle

922 between 0 and 360 degrees, apply elastic transformations ${ }^{57}$, and then crop the

923 center region of size $512 \times 512$ pixels. In addition to geometric transformations, we

924 also introduce realistic noise from experimental background images. This is done by

925 manually outlining regions of background in selected PALM training images,

926 splitting these regions into small patches of 40x40 pixels, grouping them according

927 to their summed pixel values, then assembling them into a larger image with the

928 same size as $\boldsymbol{T}$. During training, these semi-synthetic noise images are randomly

929 selected and added to the input image $\boldsymbol{S}$ without altering $\boldsymbol{T}$ or $\boldsymbol{W}$. Finally, we

930 normalize the input image $S$ by subtracting its mean and dividing by standard

931 deviation. If a widefield image $\boldsymbol{W}$ is provided, its pixel values are scaled to a 
932 minimum of 0 and a maximum of 1 . Otherwise, $\boldsymbol{W}$ is replaced by an image containing

933 zeros only. The target image $\boldsymbol{T}$ is truncated at a maximum value of 255 and then

934 scaled to have a minimum of 0 and a maximum of 1 . For the switch $\boldsymbol{M}$, we used an

935 integer number to define the type of training images, e.g. 0 for microtubules, 1 for

936 nucleoporins, 2 for mitochondria. When training on different types of images, e.g.

937 microtubules and nucleoporins (Supplementary Figure 13), we assign the

938 corresponding switch value to $\boldsymbol{M}$ and use it as additional input to the A-net together

939 with images $\boldsymbol{S}$ and $\boldsymbol{W}$, as described in Supplementary Note 1.

940

941 Image simulations

942 Our procedure to simulate localization microscopy (PALM) images of microtubules

943 is illustrated in Supplementary Figure 1. To simulate microtubule filaments, we

944 used a Langevin dynamics simulation ${ }^{29}$ that generates random configurations of

945 semiflexible curves with a specified rigidity (persistence length), starting from a

946 random initial configuration (Supplementary Figure 1a,b). The initial

947 configurations were generated with a Python library named cpolymer and the

948 Langevin dynamics was implemented using the molecular dynamics code

949 LAMMPS ${ }^{58}$. Although the simulation generates 3D polymer chains, we only

950 considered their 2D projections, consisting of $N_{p}$ connected positions $\left(x_{k}, y_{k}\right)_{k=1 . . N_{p}}$.

951 To obtain smooth filaments we further interpolated these connected segments using

952 spline functions with the Scipy function scipy.interpolate.splev. Next, we turned

953 these 2D curves into a grey scale image of $800 x 800$ pixels, with an assumed pixel

954 size of $7.8 \mathrm{~nm}$, using the Python library Matplotlib. This image was further 
955 convolved with a Gaussian kernel of standard deviation 1.5 pixels, resulting in a 956 smooth image $I(i, j)$ as shown in Supplementary Figure 1c, and normalized to a 957 probability density $\left(\sum \sum_{i, j} I(i, j)=1\right.$, with all $\left.I(i, j) \geq 0\right)$. This image was used to 958 mimic a "perfect" PALM image of filaments corresponding to an infinite number of 959 localizations $(n=\infty)$. Such perfect images were used as targets during ANN 960 training for simulated data and defined as ground truth for the quantification of 961 reconstruction quality by MS-SSIM (Figure 2g). During training, we applied the 962 same rotations and elastic transformations described for experimental data in the 963 previous section.

964 Localization microscopy images obtained from a finite number of 965 localizations $n<\infty$ (sparse PALM images $\boldsymbol{S}$ ), can be considered as a sampling of the 966 probability density $I(i, j)$ with $n$ samples. These images can therefore be simulated 967 by applying Poisson noise to a rescaled version of the perfect PALM image, i.e.: $968 \boldsymbol{S}(\lambda, I)=\mathcal{P}\left(\lambda I / I_{\max }\right)$, where $I_{\max }$ is the maximum value of $I, \mathcal{P}(\mu)$ denotes the 969 Poisson probability distribution of mean $\mu$ and where the peak parameter $\lambda$ controls 970 the level of sampling. In order to simulate sparse PALM images for various levels of 971 sampling, we varied the peak value $\lambda$ following a log-normal distribution where $972 \ln (\lambda)$ has mean -0.5 and standard deviation 0.001 and applied Poisson noise using 973 the numpy library function random.poisson. An example of a simulated sparse 974 PALM image is shown in Supplementary Figure 1d. Besides finite sampling, 975 localization microscopy images are corrupted by additional noise sources such as 976 false detections from background noise due to out-of-focus light or unspecific 977 binding of antibodies. To mimic this, we first created a probability density 
$978 I_{b}=I * G_{\sigma_{b}}$ for the background noise by convolving $I$ with a Gaussian kernel $G_{\sigma_{b}}$ of 979 large standard deviation $\sigma_{b}=25$ pixels, and applied Poisson noise with $\lambda=0.06$. To 980 create training images, we added this background noise image to the sparse PALM 981 image $\boldsymbol{S}$ above (Supplementary Figure 2). We did not add background noise to the 982 test images used during inference (Figure 2). To simulate the widefield images 983 (Figure 2a), we first blurred the perfect PALM image by convolution with a 984 Gaussian kernel of standard deviation 8 pixels, then added Gaussian noise with zero 985 mean and standard deviation chosen randomly between 0.5 and 1.5 .

986 For simulations of nuclear pore images (Supplementary Figure 15), we 987 applied a similar procedure, except that the perfect PALM images were obtained by 988 randomly distributing circles of diameter $150 \mathrm{~nm}$ in the plane (avoiding overlaps) 989 and placing eight Gaussian spots (of standard deviation 1.7 pixels) at equal distance 990 from each other on each circle to mimic the octogonal shape of nuclear pores.

991

\section{Sample preparation}

993 For microtubule imaging experiments (Figures 3-5 and Supplementary Figures 8994 10), except those using DNA-PAINT (Figure 5j,I), U-373 MG (Uppsala) cells were 995 cultured in Dulbecco's Modified Eagle Medium: Nutrient Mixture F-12 (DMEM/F12; 996 Gibco) supplemented with 10\% (v/v) fetal bovine serum (FBS; Gibco), 1\% (v/v) 997 penicillin-streptomycin (Gibco), in a $5 \% \mathrm{CO}_{2}$ environment at $37^{\circ} \mathrm{C}$ on $18-\mathrm{mm}$ 998 cleaned coverslips in 12-well plates. 24 hours after plating, cells were pre-extracted 999 for $10 \mathrm{~s}$ in $0.25 \%$ (v/v) Triton X-100 (Triton) in BRB80 (80 mM PIPES, $1 \mathrm{mM} \mathrm{MgCl2}$, 10001 mM EGTA, adjusted to pH 6.8 with $\mathrm{KOH}$ ) supplemented with 4 mM EGTA, and 
1001 immediately fixed for 10 min with $0.25 \%(\mathrm{v} / \mathrm{v})$ Triton $+0.5 \%$ Glutaraldehyde in 1002 BRB80, followed by reduction for 7 min with $0.1 \%$ NaBH4 solution in PBS and 1003 another washing step in PBS. Cells were directly incubated for $1 \mathrm{~h}$ at room 1004 temperature in PBS with 1:500 rat alpha-tubulin antibodies (Bio-Rad MCA77G), 1005 followed by 3 washing steps with PBS, and then incubated for 45min in PBS with 1006 1:500 anti-rat Alexa-647 conjugated secondary antibodies from donkey (Jackson 1007 ImmunoResearch Laboratories, ref. 712-605-153).

1008 For the DNA-PAINT experiment on microtubules (Figure 5k-m), U-373 cells 1009 stuck on $18 \mathrm{~mm}$ diameter coverslips were fixed at $37^{\circ} \mathrm{C}$ with $4 \%$ PFA in PHEM 1010 buffer and permeabilized in $0.2 \%$ glutaldehyde. Next, cells were incubated for $1 \mathrm{~h}$ 1011 with 1:500 primary mouse antibodies against alpha-tubulin. The sample was 1012 washed 3 times in PBS, then incubated with 1:100 anti-mouse oligo-conjugated 1013 antibodies from Ultivue Kit 2 for DNA-PAINT imaging ${ }^{33}$. After washing the sample 3 1014 times in PBS, and just before imaging, 2nM of complementary oligos coupled to Cy3 1015 fluorophores were added to the sample.

1016 Nuclear pore imaging data of gp210 and WGA (Figure 6a-c and 1017 Supplementary Figure 14) were kindly provided by J. Sellés and O. Falklaris and 1018 obtained from nuclear membranes of Xenopus frog eggs prepared as described 1019 previously 36.

1020 For mitochondria imaging experiments (Figure 6e-f), COS7 cells were 1021 cultured under the same conditions as U-373 cells above using phenol-red free 1022 DMEM medium and fixed with 4\% PFA in PBS for $10 \mathrm{~min}$. The sample was blocked 1023 with 3\% BSA in PBS for 20 min and immunostained with 1:500 mouse antibodies 
1024 against TOM22 (Sigma, ref. T6319) in wash buffer (PBS with 0.5\% BSA) for $1 \mathrm{~h}$.

1025 After extensive washing with wash buffer, the sample was incubated with 1:500 1026 anti-mouse secondary antibodies from donkey conjugated to Alexa-647 dyes 1027 (Jackson ImmunoResearch Laboratories, ref. 715-605-151) in wash buffer for 30 1028 min. After washing 5 times with wash buffer and 2 times with PBS, samples were 1029 post-fixed with 2\% PFA in PBS for 10 min and washed 5 times with PBS.

1030 For all localization microscopy experiments except DNA-PAINT, we used a 1031 photoswitching buffer ${ }^{59}$ composed of $50 \mathrm{mM}$ Tris- $\mathrm{HCl}+10 \mathrm{mM} \mathrm{NaCl}+10 \%(\mathrm{w} / \mathrm{v})$ 1032 glucose +168 AU/mL Glucose-Oxidase +1404 AU/mL Catalase + 1\% 21033 Mercaptoethanol. For microtubule imaging experiments, we used this buffer to fill a 1034 square hole that was manually cut in a parafilm sheet, which was deposited on a 1035 rectangular coverglass. The round coverslips were sealed with nail polish.

1036

1037 Image acquisition in localization and high-throughput microscopy

1038 We performed single molecule localization microscopy experiments (PALM/STORM 1039 and DNA-PAINT) on custom built microscopy systems, as previously described ${ }^{59-61 .}$

1040 The system used for PALM/STORM imaging of microtubules is based on an inverted 1041 microscope body (Nikon Ti Eclipse) equipped with a either a 60x 1.49 NA oil 1042 immersion objective (Nikon) or a 60x 1.2 NA water immersion objective (Nikon) 1043 and with the Perfect Focus System active. A $642 \mathrm{~nm}$ wavelength laser with $500 \mathrm{~mW}$ 1044 power was used to excite Alexa-647 fluorophores and an AOTF (AA optics) was used 1045 to modulate laser excitation. Sequences of diffraction limited single molecule image 1046 frames were acquired either on a sCMOS camera (Hamamatsu ORCA-Flash4.0), 
1047 which can capture images of 2,042x2,042 pixels (for Figures 4, 5a-i), or on an 1048 EMCCD (Andor IXON ULTRA 897) with 512x512 pixels (for Figures 3, 6d-f). Both

1049 cameras were controlled by MicroManager software ${ }^{62}$. For experiments using the 1050 sCMOS camera, the effective pixel size was $108 \mathrm{~nm}$ and we used a $512 \times 512$ region of

1051 interest, which resulted in an imaged FoV of $55.3 \mu \mathrm{m} \times 55.3 \mu \mathrm{m}$. For experiments 1052 using the EMCCD camera, we used a 2x telescope and the effective pixel size was $1053107 \mathrm{~nm}$, resulting in a FoV of $54.8 \mu \mathrm{m} \times 54.8 \mu \mathrm{m}$. The exposure time was set to $\Delta \mathrm{t}$ $1054=10 \mathrm{~ms}$ or $30 \mathrm{~ms}$ per frame. The number of frames acquired ranged from $k=1,000$ 1055 (Figure 4) to $K=60,000$ (e.g. Figure 5c) per FoV.

1056 For the DNA-PAINT experiment (Figure 5j,l), we used an inverted Nikon Ti-E 1057 Eclipse microscope equipped with a 100x 1.49 NA TIRF objective and with the 1058 Perfect Focus System active. A $561 \mathrm{~nm}$ wavelength laser with $500 \mathrm{~mW}$ power was 1059 used to excite Cy3 dyes. Highly inclined laser illumination was used to reduce out1060 of-focus background signal. Images were acquired on an EMCCD camera as above, 1061 with a $1.5 \mathrm{x}$ telescope, resulting in an effective pixel size of $106 \mathrm{~nm}$ and a FoV of $106254 \mu \mathrm{m} \times 54 \mu \mathrm{m}$. The sample was mounted in a magnetic sample holder filled with 1063 the imaging buffer provided with the Ultivue kit. Exposure time was set to $\Delta \mathrm{t}=30 \mathrm{~ms}$ 1064 and the EM gain of the EMCCD was set to 300. The laser power was increased until 1065 isolated fluorescent spots were observed. For the experiment shown in Figure 5g,i, $1066 K=60,000$ frames were acquired.

1067 The Xenopus nuclear pore data (Figure 6a-c and Supplementary 1068 Figure 14), were acquired on a Zeiss Elyra P.S.1 microscope as described 1069 previously 36. 
1071 Dimensional Acquisition tool in Micro-manager to define the positions of 1,089 FoVs 1072 of $55.3 \mu \mathrm{m} \times 55.3 \mu \mathrm{m}$ on a $33 \times 33$ grid, with overlaps of $1 \mu \mathrm{m}$; the stage was 1073 automatically shifted to each of these 1,089 positions. We first acquired only 1074 widefield images, taking five frames at each of these positions (the first two were 1075 ignored because of motion blur), in a total acquisition time of 12 minutes. Then, the 1076 laser power was raised to bleach out preactivated molecules and $k=1,000$ frames of 1077 single molecule images were acquired at each of the 1,089 positions, in a total 1078 acquisition time of 3 hours and 8 minutes. Raw image frames were written directly 1079 to a remote storage server via Samba networking protocol.

1081 Localization microscopy image analysis

1082 The input to ANNA-PALM reconstruction is a localization image, defined as a 2D 1083 histogram of $n$ single molecule positions $\left(x_{i}, y_{i}\right)_{i=1 . . n}$. The histogram bin, i.e. the 1084 pixel size of the localization image, was set to $7.8 \mathrm{~nm}$ for the simulated data 1085 (Figure 2 and Supplementary Figures 1-6,15) and $20 \mathrm{~nm}$ for the experimental 1086 data (Figures 3-6 and Supplementary Figures 7-14). The positions $\left(x_{i}, y_{i}\right)_{i=1 . . n}$ 1087 were obtained by analyzing sequences of diffraction limited frames using standard 1088 single molecule localization algorithms. For experimental microtubule images, we 1089 used the ThunderSTORM ${ }^{63}$ plugin of ImageJ, applying wavelet filters for detection 1090 and weighted least squares Gaussian fitting for precise estimation of subpixelic 1091 positions. We used the cross-correlation feature in ThunderSTORM for drift 1092 correction, and filtered out the least certain localizations based on the fitted 
1093 Gaussian's standard deviation and the $\chi^{2}$ of the residual. Localizations in 1094 consecutive frames separated by less than $20 \mathrm{~nm}$ were assumed to originate from 1095 the same molecule and merged into a single localization. The final number $n$ of 1096 localizations was $\sim 7$ million for the full $55 \mu \mathrm{m}$ x $55 \mu \mathrm{m}$ FoV of the images shown in 1097 Figure 3 and Supplementary Figures 8,9 (obtained from $K=30,000$ frames). For 1098 the high-throughput experiment (Figure 4 and Supplementary Figure 12), the 1099 number of localizations per $55 \mu \mathrm{m} \times 55 \mu \mathrm{m}$ FoV ranged from $n=2,949$ to $1100 n=1,442,048$ with an average $\langle n\rangle=610,983$ and standard deviation $\sigma(n)=273,606$.

1101 The total number of localizations across all 1,089 FoVs was $\approx 665$ million.

1102 ThunderSTORM analyses were performed either on high end workstations or on 1103 Institut Pasteur's high performance computer (HPC) cluster. For the high1104 throughput experiments, we used Python scripts to run ThunderSTORM in batch 1105 mode (without user intervention) on the HPC cluster and assembled mosaic images 1106 (Figure 4a,b and Supplementary Figure 12a,b) using a stitching plugin of 1107 Image ${ }^{64}$.

1108 The nuclear pore images were analyzed using the ZEN software from Zeiss as 1109 previously described ${ }^{36}$. For the DNA-PAINT experiments, we used PALMTT, a 1110 modified version of the single molecule tracking algorithm MTT65, based on Matlab

1111 (Mathworks). This algorithm uses Gaussian smoothing and thresholding for 1112 detection, and Gaussian fitting for precise estimation of subpixelic positions. Drift 1113 correction was performed computationally by tracking fluorescent beads used as 1114 fiducial markers. 


\section{Quality metrics and sampling resolution}

1117 In order to quantitatively assess the quality of PALM images and ANNA-PALM

1118 reconstructions, we calculated the multi-scale structural similarity index (MS-SSIM)

1119 between either image and the ground truth (Figure 2g and Supplementary

1120 Figures 5,9). For the simulated data, the ground truth was simply defined as the

1121 "perfect" PALM image, corresponding to an infinite number of localizations (see

1122 'Image simulations' above, Figure 2e and Supplementary Figure 1c). For the

1123 experimental data, the ground truth was defined as the ANNA-PALM reconstruction

1124 of a dense PALM image obtained from all available frames (e.g. Supplementary

1125 Figure 9c). Before calculation of the MS-SSIM, all simulated images were linearly

1126 normalized without clipping to a maximum value of 255.

1127 In order to evaluate the effect of sampling on the resolution of PALM images,

1128 we computed the double mean distance, $R_{\mathrm{Nyq}}=2\langle d\rangle$ between nearest neighbors in

1129 the underlying sets of localizations ${ }^{49}$ as function of localization number

1130 (Supplementary Figure 3d). For the simulated sparse PALM data, sets of

1131 localizations were obtained by interpreting each image $\boldsymbol{S}$ as a 2D histogram of

1132 localizations, and creating a random subpixelic position $\left(x_{k}, y_{k}\right)_{k=1 . . m}$ within each

1133 pixel $(i, j)$, as many times as given by the pixel value $m=S(i, j)$ (therefore resulting

1134 in a set of $n=\sum_{i, j} S(i, j)$ localizations). The quantity $R_{\mathrm{Nyq}}(n)=2\langle d(n)\rangle$ decreases

1135 towards zero with increasing number of localizations, $n$. A Nyquist criterion

1136 introduced in ref. ${ }^{49}$ suggests that the resolution is limited by sampling to no less

1137 than $R_{\mathrm{Nyq}}$, implying that at least $n \geq N_{\mathrm{Nyq}}=R_{\mathrm{Nyq}}^{-1}(R)$ localizations are needed to

1138 achieve a given resolution $R$. However, a more stringent and realistic criterion ${ }^{3}$ 
1139 prescribes a five-fold larger number of localizations to reach resolution $R$ :

$1140 n \geq N_{5 \times \mathrm{Nyq}}=5 \times N_{\mathrm{Nyq}}$, implying that the sampling limit to resolution is

$1141 R_{5 \times \mathrm{Nyq}}=2\langle d(n / 5)\rangle$. Accordingly, if this condition is met, the resolution is no longer

1142 limited by sampling, but by the localization precision, $R_{\mathrm{loc}} \approx 2.3 \sigma_{\mathrm{loc}}$, where $\sigma_{\mathrm{loc}}$ is

1143 the standard deviation of localization errors along each coordinate. In general, the

1144 resolution of a PALM image, as limited by both sampling and localization precision,

1145 can be written: $R=\max \left(R_{5 \times \mathrm{Nyq}}, R_{\mathrm{loc}}\right)=\max \left(2\langle d(n / 5)\rangle, 2.3 \sigma_{\mathrm{loc}}\right)$.

1147 Life Sciences Reporting Summary.

1148 Further information is available in the Life Sciences Reporting Summary.

1150 Code availability statement

1151 The source code of ANNA-PALM is available from https://annapalm.pasteur.fr/.

\section{Data availability statement}

1154 The localization data used in this paper can be downloaded directly from 1155 https://annapalm.pasteur.fr/.

1157 Methods-only references

1158 51. Xu, L., Choy, C. S. \& Li, Y. W. Deep sparse rectifier neural networks for speech

1159

1160 denoising. in 2016 International Workshop on Acoustic Signal Enhancement, IWAENC 2016 (2016). doi:10.1109/IWAENC.2016.7602891

1161

1162

1163

1164

52. Maas, A. L., Hannun, A. Y. \& Ng, A. Y. Rectifier Nonlinearities Improve Neural Network Acoustic Models. Proc. 30 th Int. Conf. Mach. Learn. (2013).

53. Mao, X. et al. Least Squares Generative Adversarial Networks. in ICCV 27942802 (2017). doi:10.1109/ICCV.2017.304 
1165

1166

1167

1168

1169

1170

1171

1172

1173

1174

1175

1176

1177

1178

1179

1180

1181

1182

1183

1184

1185

1186

1187

1188

1189

1190

1191

1192

1193

1194

1195

1196

1197

1198

1199

1200

54. Kingma, D. P. \& Ba, J. Adam: A Method for Stochastic Optimization. ICLR 1-15 (2015).

doi:http://doi.acm.org.ezproxy.lib.ucf.edu/10.1145/1830483.1830503

55. Rampasek, L. \& Goldenberg, A. TensorFlow: Biology's Gateway to Deep Learning? Cell Syst. 2, 12-14 (2016).

56. Srinivasa Reddy, B. \& Chatterji, B. N. An FFT-based technique for translation, rotation, and scale-invariant image registration. IEEE Trans. Image Process. 5, 1266-1271 (1996).

57. Simard, P. Y., Steinkraus, D. \& Platt, J. C. Best practices for convolutional neural networks applied to visual document analysis. Seventh Int. Conf. Doc. Anal. Recognition, 2003. Proceedings. 1, 958-963 (2003).

58. Plimpton, S. Fast Parallel Algorithms for Short-Range Molecular Dynamics. J. Comput. Phys. 117, 1-19 (1995).

59. van de Linde, S. et al. Direct stochastic optical reconstruction microscopy with standard fluorescent probes. Nat. Protoc. 6, 991-1009 (2011).

60. Henriques, R. et al. QuickPALM: 3D real-time photoactivation nanoscopy image processing in ImageJ. Nat. Methods 7, 339-340 (2010).

61. Lelek, M. et al. Superresolution imaging of HIV in infected cells with FlAsHPALM. Proc. Natl. Acad. Sci. U. S. A. 109, 8564-9 (2012).

62. Edelstein, A., Amodaj, N., Hoover, K., Vale, R. \& Stuurman, N. Computer control of microscopes using manager. Current Protocols in Molecular Biology (2010). doi:10.1002/0471142727.mb1420s92

63. Ovesny, M., Kiek, P., Borkovec, J., Vindrych, Z. \& Hagen, G. M. ThunderSTORM: a comprehensive ImageJ plug-in for PALM and STORM data analysis and super-resolution imaging. Bioinformatics 30, 2389-2390 (2014).

64. Preibisch, S., Saalfeld, S. \& Tomancak, P. Globally optimal stitching of tiled 3D microscopic image acquisitions. Bioinformatics 25, 1463-1465 (2009).

65. Sergé, A., Bertaux, N., Rigneault, H. \& Marguet, D. Dynamic multiple-target tracing to probe spatiotemporal cartography of cell membranes. Nat Methods 5, 687-694 (2008). 


\section{ANNA-PALM: Deep learning accelerates super-resolution localization}

2 microscopy by orders of magnitude

3

4

5 Wei Ouyang $1,2,3$, Andrey Aristov ${ }^{1,2,3}$, Mickaël Lelek ${ }^{1,2,3}$, Xian Hao ${ }^{1,2,3}$, Christophe

6 Zimmer ${ }^{1,2,3}$

7

$8{ }^{1}$ Institut Pasteur, Unité Imagerie et Modélisation, Paris, France

$9 \quad 2$ UMR 3691, CNRS

$10 \quad{ }^{3}$ C3BI, USR 3756, IP CNRS

11

12

13

14

15

16

17

18

19

20

21

22 Corresponding author: C. Zimmer 
23 The speed of super-resolution microscopy methods based on single molecule

24 localization, e.g. PALM or STORM, is severely limited by the need to record

25 many thousands of frames with a low number of observed molecules in each.

26 Here, we present ANNA-PALM, a computational strategy that uses artificial

27 neural networks to reconstruct super-resolution views from sparse, rapidly

28 acquired localization images and/or widefield images. Simulations and

29 experimental imaging of microtubules, nuclear pores and mitochondria show

30 that high-quality super-resolution images can be reconstructed from up to two

31 orders of magnitude fewer frames than usually needed, without

32 compromising spatial resolution. Super-resolution reconstructions are even

33 possible from widefield images alone, though adding localization data

34 improves image quality. We demonstrate super-resolution imaging of $>\mathbf{1 , 0 0 0}$

35 fields of view containing $>\mathbf{1 , 0 0 0}$ cells in $\sim 3 \mathrm{~h}$, yielding an image spanning

36 spatial scales from $\sim 20 \mathrm{~nm}$ to $\sim 2 \mathrm{~mm}$. The drastic reduction in acquisition

37 time and sample irradiation afforded by ANNA-PALM enables faster and

38 gentler high-throughput and live cell super-resolution imaging. 
40 Fluorescence microscopy methods that overcome the diffraction limit of resolution

41 ( 200-300 nm) allow imaging of biological structures with molecular specificity 42 closer to the molecular scale. Among super-resolution microscopy approaches,

43 those based on single molecule localization, such as PALM ${ }^{1}$ or STORM ${ }^{2}$ (hereafter

44 referred to collectively as PALM) are particularly attractive owing to their exquisite

45 spatial resolution and ease of implementation. In these methods, random subsets of

46 fluorophores are imaged in many consecutive diffraction-limited frames,

47 computationally localized to high precision, and the combined localizations are used

48 to generate a super-resolution view. In practice, typically $10^{3}-10^{5}$ diffraction-limited

49 frames are needed to assemble a single super-resolution image. This requirement

50 follows from two conditions that must be simultaneously satisfied to ensure high

51 spatial resolution: (i) a low number $\left(\sim 10-10^{2}\right)$ of active fluorophores per frame, to

52 avoid overlaps between diffraction limited spots and enable precise localization of

53 individual molecules, and (ii) a large number of independent localizations to ensure

54 a sufficiently dense sampling of the underlying biological structures ${ }^{3,4}$. The large

55 number of required frames makes localization microscopy inherently slow, thereby

56 limiting its potential for high-throughput imaging, where many fields of view (FoVs)

57 are to be imaged, and for imaging live cell dynamics. As a result, most localization

58 microscopy studies are restricted to analyzing a small number of cells (typically less

59 than ten).

60 Multiple approaches have been explored to accelerate localization

61 microscopy. Using bright dyes with rapid switching kinetics, high power lasers and

62 fast cameras allows to minimize exposure time without losing signal to noise 
63 ratio 5,6 , but reaching sub-millisecond exposure remains challenging, and intense

64 irradiation exacerbates phototoxicity in live cell imaging ${ }^{7,8}$. Increasing the number

65 of active fluorophores per frame can reduce acquisition time, but despite algorithms

66 designed to handle overlapping fluorescent spots $^{9-13}$ this approach necessarily

67 compromises spatial resolution ${ }^{14,15}$.

68 Here, we introduce a computational strategy that allows reducing the total

69 number of frames and independent localizations without losing spatial resolution:

70 Unlike previous approaches, our method leverages the structural redundancy of

71 most biological images to reconstruct high quality images from vastly under-

72 sampled localization microscopy data. Our method leverages deep learning, which

73 employs artificial neural networks (ANNs) to learn complex non-linear mappings

74 between numerical inputs and outputs ${ }^{16}$. Accordingly, we call it 'artificial neural

75 network accelerated PALM', or ANNA-PALM.

76

77 RESULTS

78 A deep learning approach to super-resolution image reconstruction

79 We aim to reconstruct a super-resolution image of approximately similar 80 information content as a standard PALM acquisition (with $K$ frames and $N$

81 localizations) from a much smaller number of raw frames $(k<<K)$ without changing

82 the average density of localizations, $\rho$, i.e. from a much smaller number of total

83 localizations $(n=\rho k \ll N=\rho K)$. If PALM images are defined as 2D histograms of

84 independent localizations, this task can be formulated as restoring an image

85 corrupted by Poisson noise (and potentially additional forms of noise). Image 
86 restoration is an ill-posed problem that has an infinity of solutions in the high-

87 dimensional space of all possible images, unless additional constraints (priors) are

88 imposed that restrict the solution to a lower dimensional subspace. Suitable

89 subspaces exist because most natural images are highly redundant, and can be

90 represented to very good approximation with a much smaller number of coefficients

91 than pixels, via appropriate functions that map feature space to pixel space ${ }^{17,18}$. In

92 recent years, ANNs with multiple layers (deep nets) have proven very successful at

93 learning meaningful features and non-linear mappings for image classification,

94 segmentation, restoration and many other tasks ${ }^{16,18,19}$. Inspired by these

95 developments, we designed ANNA-PALM, a deep learning approach for restoring

96 super-resolution views from under-sampled (sparse) localization microscopy data.

97 ANNA-PALM comprises a training stage and an inference stage (Figure 1).

98 For training (Figure 1a), a few super-resolution images representative of the

99 structure of interest (e.g. microtubules, nuclear pores, or mitochondria) are

100 obtained using standard PALM imaging, i.e. by acquiring long diffraction limited

101 image sequences (e.g. $K \sim 10^{4}-10^{5}, N \sim 10^{5}-10^{7}$ ) and processing them with

102 standard localization software ${ }^{20}$, resulting in highly sampled (dense) PALM images.

103 In addition, a low resolution (widefield) image can also be acquired, as is commonly

104 done before single molecule imaging when bleaching out preactivated fluorophores.

105 Next, the dense PALM images are under-sampled by using a much smaller number

106 of input frames, $k \ll K$, thus yielding sparse PALM images from the same

107 localization data. Then, an ANN is trained to recover approximations of the dense

108 PALM images from these sparse PALM images (and the optional widefield image). 
109 Once trained, the ANN is applied to new sparse PALM images (with or without a

110 widefield image), obtained from new image sequences with small numbers of

111 frames $(k \ll K)$-and hence in much shorter time- in order to reconstruct high

112 quality super-resolution images not previously seen (inference, Figure 1b).

113

\section{Neural net architecture and learning strategy}

115 Our ANN, hereafter called A-net, contains a total of 25 convolutional layers, and

116 roughly 42 million trainable parameters. A-net is adapted from the pix2pix

117 network ${ }^{21}$, which itself builds on U-nets ${ }^{22}$ and generative adversarial networks

118 (GANs) ${ }^{23}$, two recent successful deep learning techniques. U-nets are special types of

119 convolutional neural networks (CNNs) that have proven effective at learning multi-

120 scale representations of images and accurate, pixel-wise mappings ${ }^{22,24}$. GANs can

121 generate new samples from real image distributions using a generator network that

122 outputs synthetic images, and a discriminator network that outputs the probability

123 that an input image is real or synthetic, both networks being trained simultaneously

124 to compete against each other ${ }^{23}$. Importantly, the generator can be conditioned on

125 input data (conditional GAN, or cGAN) ${ }^{21,23}$, e.g. on images as in the pix2pix network.

126 We modified the pix2pix architecture to accept a computational switch as additional

127 input to handle multiple types of data, and introduced an additional network to

128 evaluate the consistency between the reconstructed image and the widefield input

129 image.

130 Training of our A-net proceeds as follows. Randomly under-sampled (i.e.

131 sparse) versions of PALM images are fed as input to the A-net, while the 
132 corresponding dense PALM images are defined as the A-net's targets, i.e. desired

133 outputs (Figure 1a). Additional, optional inputs are widefield images, if available,

134 and the switch, which indicates the image type when multiple types of images (e.g.

135 microtubules and nuclear pores) are used during training. ANN training requires

136 defining an objective function (also called loss), which measures how well the

137 outputs match the targets. We implemented a loss function containing three terms.

138 The first term measures the difference between the A-net output and the dense

139 PALM image. Instead of the widely used mean squared error (MSE), which poorly

140 reflects visual quality25, we used a combination of the absolute difference (L1 norm)

141 with a multi-scale version of the structural similarity index, a perceptually

142 motivated quality metric shown to improve image restoration with deep learning

143 (MS-SSIM) ${ }^{26}$. The second term measures the consistency between the A-net output

144 and the widefield image. Although in theory the latter should simply be a blurred

145 version of the PALM image, this is often not the case in practice ${ }^{27}$. Therefore, we

146 introduced another CNN (with 4-layers), called 'low resolution estimator' to predict

147 the widefield image from the super-resolution image. The corresponding loss was

148 defined as the MS-SSIM between this CNN's output and the observed widefield

149 image. (In absence of a widefield image, this loss is set to zero). The third term

150 contains a cGAN discriminator $\operatorname{loss}^{21}$, where the discriminator is a 5-layer CNN,

151 whose inputs are the sparse PALM (and widefield) image(s) and either the dense

152 PALM image or the output of the generator above; the discriminator's output is

153 compared to 0 s and 1 s (for synthetic and real, respectively), respectively, via the

154 MSE. We use dropout ${ }^{28}$ and extensive data augmentation, including random 
155 rotations, translations, elastic deformations and addition of noise in the input image

156 to mimic false detections and unspecific labeling. As a result, only a few dense PALM

157 images are required for successful training without overfitting. On graphical 158 processing units (GPU), training ANNA-PALM from scratch takes on the order of

159 hours to days, but when starting from a previously trained A-net, retraining can be 160 done in an hour or less.

161 Once trained, the A-net can take sparse localization data with an optional 162 widefield image as input(s), and output a reconstructed super-resolution image in

163 less than a second (Figure 1b). In addition, the A-net produces an 'error map' that 164 measures the consistency of this super-resolution image with the widefield image ${ }^{27}$

165 (when available) and can be used to estimate the degree of reliability and highlight 166 potential reconstruction artifacts. For more details, see Online Methods and

\section{Supplementary Note 1.}

\section{Validating ANNA-PALM on simulated images}

170 We first sought to validate ANNA-PALM on synthetic data. For this, we used

171 Brownian dynamics simulations ${ }^{29}$ to generate 200 dense PALM images of semi-

172 flexible filaments mimicking microtubules, with a resolution $R_{\text {loc }} \approx 23 \mathrm{~nm}$. These

173 represent "perfect" PALM images that would be obtained with an infinite number of

174 localizations. We applied varying levels of Poisson noise to these perfect images to

175 create sparse PALM images corresponding to finite numbers of localizations

176 (Supplementary Figure 1). We then trained our A-net using the perfect images as 
177 targets and the sparse images (and widefield image) as inputs, varying the number

178 of localizations over a large range.

179 Next, we applied the trained A-net to a distinct set of PALM images generated

180 by the same stochastic simulation (Supplementary Figure 2). Figure 2a shows a

181 widefield image and Figure $\mathbf{2 b}$ a corresponding sparse PALM image obtained from

$182 n=6,834$ localizations. Although curvilinear structures can be seen in this image

183 despite its sparsity, small-scale features remain highly ambiguous (Figure $\mathbf{2 b}$,

184 inset), and the resolution according to a recently proposed five-fold Nyquist

185 criterion $^{3}$ is limited by sampling to $R_{5 \times \mathrm{Nyq}} \approx 85 \mathrm{~nm}$; according to this criterion,

$186 N>N_{5 \times N y q}=60,000$ localizations are needed to achieve $23 \mathrm{~nm}$ resolution

187 (Supplementary Figure 3d). Figure 2c shows the ANNA-PALM image

188 reconstructed from the wide-field image alone, which exhibits clear and continuous

189 filaments that were not previously recognizable. Most of the relatively isolated

190 filaments roughly agree with the perfect PALM image (Figure 2e). In the denser

191 regions, however, many small features are erroneous, e.g. filaments are incorrectly

192 joined, displaced, split or merged (Figure 2c, blue arrows and Supplementary

193 Figure 4, top). By contrast, the ANNA-PALM image reconstructed from the sparse

194 PALM image alone or in combination with the widefield image exhibits continuous

195 and sharp filaments in very good agreement with the perfect PALM image

196 (Supplementary Figure 3b,c and Figure 2d,e,f). The spatial resolution of these

197 reconstructed images is limited neither by diffraction nor sampling, but only by the

198 localization precision, and is thus $R_{\mathrm{loc}} \approx 23 \mathrm{~nm}$, as in the perfect images

199 (Supplementary Figure 3e,f). These results indicate that high quality super- 
200 resolution images can be obtained from only a small fraction of the number of

201 localizations traditionally required (here, approximately $\sim 11 \%$ of $N_{5 x N y q}$ above; see

202 Supplementary Figure 3d), hence enabling a strong reduction in acquisition time.

203 Nevertheless, reconstruction errors can still occur in areas where the sparse

204 localization data are most ambiguous, e.g. where filament density is highest

205 (Figure 2d,e,f, white arrow). These errors can be reduced by increasing the

206 localization number $n$, implying a trade-off between acquisition time and 207 reconstruction quality (Supplementary Figure 4).

208 To quantify this trade-off, we computed the MS-SSIM between reconstructed 209 ANNA-PALM and perfect PALM images $(n=\infty)$ as function of localization number, 210 from $n \sim 200$ to $n \sim 2 \times 10^{6}$, in comparison with the standard PALM images 211 (Figure 2g). The MS-SSIM ranges from 0 to 1 and reaches 1 for perfect 212 reconstructions. For standard PALM images, the MS-SSIM increases monotonically, 213 as expected, from $<0.2$ to $>0.95$ for $n=2 \times 10^{6}$ million localizations (Figure 2g, black 214 curve). Using only the sparse image as input, ANNA-PALM reconstructions achieve 215 MS-SSIM that are consistently higher and increase with localization number $n$ much 216 more rapidly than standard PALM, already exceeding 0.9 for $n \approx 10,000$ 217 localizations (Figure 2, dashed blue curve). ANNA-PALM achieves the same MS218 SSIM as standard PALM at the five-fold Nyquist sampling level $(\approx 0.65)$ with only $219 n=2,248$ localizations instead of $n=58,588$, suggesting a $\sim 26$-fold speed-up. If the 220 widefield image is used as additional input, the MS-SSIM further increases, and 221 dramatically so for low localization numbers (Figure 2g, solid blue curve). For 222 example, with $n=7,477$ localizations, ANNA-PALM achieves a MS-SSIM $(\approx 0.95)$ 
223 similar to standard PALM with $n=644,844$, implying a speed-up of roughly two

224 orders of magnitude. (Note that, if the perfect PALM image was not available for

225 these quantifications, it could be replaced by the ANNA-PALM reconstruction of a

226 dense PALM image with a large number of localizations, e.g. $n=10^{5}$, with similar

227 results- see Supplementary Figure 5).

228 As any image restoration method, ANNA-PALM can make errors. The low

229 resolution error map described above (Figure 1b) provides a means to estimate

230 where errors are most likely to occur. When applied to ANNA-PALM reconstructions

231 of a sparse PALM image, this error map highlights regions containing the highest

232 density of filament crossings, where reconstructions tend to be least accurate

233 (Supplementary Figure 6i,k). If we artificially displace a small piece of filament in

234 this image to simulate a false positive and a false negative in the reconstruction

235 (Supplementary Figure 6b,d, white and blue arrows, respectively), the affected

236 regions also light up in the error map (Supplementary Figure 6j,1). Thus, the error

237 map offers a useful tool to highlight regions most likely to contain reconstruction

238 errors, and conversely, to outline regions where reconstructions are most

239 trustworthy. Thus, simulations suggest that ANNA-PALM can considerably reduce

240 acquisition time in localization microscopy and also map reconstruction reliability.

\section{ANNA-PALM reconstructions of immunostained microtubules}

243 We next tested our method on real images of immunolabeled microtubules

244 (Figure 3). We trained our A-net on seven dense PALM images (with corresponding

245 widefield images) obtained during 10 minute long acquisitions $(K=60,000$; 
$246 \Delta \mathrm{t}=10 \mathrm{~ms}$ exposure time) (not shown). We then considered a sparse PALM image of

247 microtubules in a distinct FoV obtained from only $9 \mathrm{~s}$ of acquisition $(k=300 ; \Delta \mathrm{t}=30$

$248 \mathrm{~ms})$, together with a widefield image $(\Delta \mathrm{t}=2 \times 50 \mathrm{~ms}$ ) (Figure 3a,b). Whereas

249 microtubule filaments can already be seen in this sparse PALM image, structural

250 details below the diffraction limit are hard to discern, making it difficult to follow

251 the path of individual filaments in the denser regions and to identify features such

252 as filament crossings (Figure 3b). By contrast, the ANNA-PALM images, whether

253 reconstructed from the widefield image alone, the sparse PALM image alone, or

254 both, all display sharp and continuous filaments and clearly reveal many structural

255 details (Figure 3d-f). Their resolution is similar to or even better than the dense

256 PALM image (Supplementary Figure 7a). As for the simulations, in regions where

257 microtubule filaments are isolated, the ANNA-PALM image reconstructed from the

258 widefield image alone is in good agreement with the dense PALM image

259 (Figure 3d,g). However, it is often incorrect in areas of high microtubule density

260 (e.g. Figure 3d,g white and gray arrows). Most of these reconstruction errors are

261 corrected when applying ANNA-PALM to the sparse PALM image instead

262 (Figure 3e,h). For example, parallel sections of two microtubules unresolved in the

263 widefield image and incorrectly merged in Figure 3d are now clearly separated and

264 positioned correctly, and missed portions of other filaments are now recovered

265 (Figure 3h, white and gray arrows). Counter-intuitively, the sparse PALM image

266 exhibits high signal in some locations where the dense PALM image does not,

267 presumably because of spurious localizations due e.g. to unspecific binding

268 (Figure 3b, blue arrow). Such signal can lead to incorrect features in the ANNA- 
269 PALM reconstruction from the sparse localization data alone (Figure 3e,h, blue 270 arrows). However, when combining the widefield and sparse PALM data, these 271 artifacts are largely removed and reconstructions agree very well with the dense 272 PALM image (Figure 3f,i). Reconstruction quality increases with the number of 273 frames $k$ (Figure 3j, Supplementary Figures 8-9 and Supplementary Video 1). 274 More quantitatively, a MS-SSIM analysis similar to that for the simulated data above 275 (with the ANNA-PALM output of the dense PALM image defined as ground truth; see 276 Supplementary Figures 5, 9f) suggests that ANNA-PALM allows a hundred-fold 277 reduction of acquisition time compared to standard PALM (Supplementary 278 Figure 9g). Supplementary Figure 10 shows other examples of sparse 279 microtubule images reconstructed by ANNA-PALM.

280 As for simulations above, we used the widefield image to compute an error 281 map (Supplementary Figures 6,11). Bright areas in this error map highlight 282 regions where the reconstruction indeed disagrees with the dense PALM image; 283 conversely, reconstructions are of high quality in the majority of regions where the 284 error map is dim (Supplementary Figure 11d-f). These results demonstrate 285 experimentally that ANNA-PALM can restore high quality approximations of super286 resolution images from much shorter acquisition time than typical for PALM 287 imaging, and also predict where reconstruction errors are most likely.

289 ANNA-PALM enables high-throughput super-resolution imaging

290 The drastic improvement in imaging efficiency afforded by ANNA-PALM permits 291 super-resolution imaging of orders of magnitude more cells and FoVs per unit time. 
292 To demonstrate this, we used an automated acquisition protocol to image $>1,000$ 293 cells with immunolabeled microtubules in 1,089 (33x33), partly overlapping, FoVs 294 of $55.3 \mu \mathrm{m} \times 55.3 \mu \mathrm{m}$ each (Figure 4, Supplementary Figure 12). We first acquired 295 widefield images at each of these positions, in a total of $\sim 12$ minutes, mostly 296 consisting of stage stabilization delays (Supplementary Figure 12a). Next, we 297 obtained 1,089 sparse PALM images using only $10 \mathrm{~s}$ of imaging time per FoV 298 ( $k=1,000$ frames, $\Delta t=10 \mathrm{~ms}$ ), in a total of only $\sim 3.1$ hours (Figure 4a). Neither the 299 widefield nor the sparse PALM images provided much small scale information 300 (Figure 4c, Supplementary Figure 12 c,e). However, ANNA-PALM reconstructions 301 led to high quality super-resolution images, allowing to visualize the microtubule 302 network with clarity and to distinguish microtubule filaments in dense areas that 303 appeared as unstructured regions in the sparse PALM image (Figure $\mathbf{4 b}, \mathbf{d}$ ). The 304 FWHM across filaments in the reconstructed image was $\sim 51 \mathrm{~nm}$ (Figure 4d), within 305 the range measured for the training data (Supplementary Figure 7a). Similar 306 images can be obtained by ANNA-PALM using the widefield images alone 307 (Supplementary Figure 12), although of lower quality, as discussed above. 308 Stitching the reconstructed images together yielded a single super-resolution image 309 that contained approximately seven billion $20 \times 20 \mathrm{~nm}$ pixels and covered an area of $3101.8 \mathrm{~mm} \times 1.8 \mathrm{~mm}$, thereby spanning almost five orders of magnitude in spatial scales 311 (Figure 4b,d, Supplementary Figure 12b and Supplementary Video 2). 312

313 ANNA-PALM is robust to experimental perturbations 
314 ANNA-PALM can reconstruct accurate super-resolution images from sparse data

315 because it was trained on similar images before. This raises the question of whether

316 an ANN trained in one experimental condition can be successfully applied to

317 another condition. To test this, we used the A-net as trained above to analyze

318 microtubule images of cells subjected to drugs affecting the cytoskeletal network.

319 We first treated U373 cells with $1 \mu \mathrm{M}$ of Taxol, an antimitotic agent that inhibits the

320 depolymerization of microtubules and increases their bending rigidity ${ }^{30,31}$.

321 Figure 5d shows a sparse PALM image of these cells ( $k=800, \mathrm{k} \Delta \mathrm{t}=8 \mathrm{~s})$, in which the

322 microtubule network is barely recognizable. By contrast, the ANNA-PALM

323 reconstructions clearly display a complex microtubule network and agree well with

324 the dense PALM image obtained from $K=60,000$ frames $(K \Delta t=10 \mathrm{~min})$

325 (Figure 5d,e,f). These images display a larger density of straighter and more

326 parallel filaments with less frequent crossings than in the untreated cells

327 (Figure 5a-c), consistent with microtubule stabilization and increased rigidity ${ }^{31}$.

328 Next, we treated cells with $1 \mu \mathrm{M}$ of Nocodazole, a drug that, on the opposite,

329 promotes microtubule depolymerization and is expected to more dramatically alter

330 the cytoskeletal network ${ }^{32}$. Again, whereas the sparse image obtained from $k=300$

331 frames $(k \Delta t=3 \mathrm{~s})$ contained little exploitable information (Figure 5g), the ANNA-

332 PALM reconstruction offered clear and detailed views of the disrupted microtubule

333 network (Figure 5h), exhibiting a much smaller number of filaments, with higher

334 curvature, than in untreated cells. These reconstructions were in good (though not

335 perfect) agreement with dense PALM images obtained from $K=60,000$ frames

$336(K \Delta \mathrm{t}=10 \mathrm{~min})$ (Figure 5i). Thus, ANNA-PALM, when trained on microtubules in 
337 one experimental condition, may be successfully applied to new experimental 338 conditions without retraining, thereby highlighting the method's robustness to 339 biologically relevant structural alterations.

340 We further asked if ANNA-PALM is robust to changes in technical imaging 341 conditions. To address this, we performed localization microscopy on microtubules

342 by simultaneously changing multiple important imaging parameters relative to the

343 training data. Instead of PALM/STORM, we used DNA-PAINT a technique where

344 single molecule detection relies on transient binding of fluorophore-conjugated

345 short DNA strands to complementary, antibody-conjugated, DNA strands ${ }^{33}$, rather

346 than on fluorophore blinking. The continuously emitting freely diffusing dyes lead to

347 higher background noise in DNA-PAINT compared to PALM/STORM. Moreover, we

348 used primary mouse antibodies instead of rat antibodies, Cy3 dyes instead of Alexa-

349647 dyes, and an EMCCD instead of a sCMOS camera. Despite all these differences,

350 when ANNA-PALM was applied without retraining on a sparse microtubule image

351 ( $k=400$ frames, $\mathrm{k} \Delta \mathrm{t}=12 \mathrm{~s}$ ), the reconstructed image still agreed very well with the

352 dense DNA-PAINT image obtained from $K=60,000$ frames $(K \Delta \mathrm{t}=30 \mathrm{~min}$ ) (Figure $\mathbf{5 j}$ -

353 1). These data demonstrate the high robustness of ANNA-PALM to changes in

354 experimental imaging conditions.

355

356 ANNA-PALM adapts to different biological structures

357 To demonstrate that ANNA-PALM is not restricted to filamentary structure, we

358 turned to nuclear pores, a very different biological structure, and another popular

359 target of super-resolution imaging studies ${ }^{34-36}$. We retrained A-net simultaneously 
360 on microtubule images and on a single PALM image of the nucleoporin gp210 in

361 immunolabeled nuclear membranes of Xenopus frog eggs ${ }^{34,36}(K=30,000)$. With the

362 switch (Figure 1) set to microtubules ('MT'), this newly trained A-net can still

363 reconstruct sparse images of microtubules as when trained exclusively on

364 microtubule data (Supplementary Figure 13a-c). We then applied the same A-net

365 with the switch set to nuclear pores ('NPC') to reconstruct a new sparse PALM

366 image of gp210 obtained from the first $k=3,000$ frames (Figure 6a). The sparsity of

367 this image makes it difficult to clearly distinguish individual nuclear pores. ANNA-

368 PALM, however, reconstructs a much clearer image, containing many easily

369 identifiable ring-like structures, as expected for nuclear pores ${ }^{34}$ (Figure 6b), and in

370 good agreement with the dense PALM image obtained from $K=30,000$ frames (even

371 though the latter shows mostly incomplete, open rings, presumably due to

372 suboptimal labeling) (Figure 6c). An automated procedure based on cross-

373 correlation with a ring template indeed identified $\sim 2.7$ times more putative nuclear

374 pores from the ANNA-PALM image than the sparse image (Supplementary Figure

375 14a-c). Moreover, computed pore locations were in good agreement with a PALM

376 image of wheat germ agglutinin (WGA), a lectin that concentrates in the inner

377 nuclear pore channel ${ }^{36}$ (Supplementary Figure 14 d-f). These results show that

378 ANNA-PALM can successfully analyze non-filamentary structures, when properly

379 retrained, and that a single ANN, with a simple computational switch, can

380 reconstruct very different types of structures.

381 Finally, we imaged TOM22, a protein of the mitochondrial outer membrane ${ }^{37}$.

382 Whereas, at the resolution of our experiments, microtubules and nucleoporins are 
383 essentially one-dimensional and zero-dimensional structures, mitochondrial

384 membranes are two-dimensional surfaces. Furthermore, their complex 3D

385 morphology might seem less predictable than filaments or nuclear pores, potentially

386 hampering ANNA-PALM reconstruction. Despite these differences, after being

387 trained on nine PALM images of TOM22 (with frame numbers ranging from

$388 K=24,000$ to $K=40,000$ ), ANNA-PALM reconstructions of distinct sparse PALM

389 images ( $k=400$ frames) displayed mitochondrial morphologies in good agreement

390 with the dense PALM images (Figure 6d-f) - although the protein's localization

391 along the membrane was less well reproduced. Taken together, our results illustrate

392 the versatility of ANNA-PALM and its applicability to images of very different

393 structural content.

394

\section{Discussion}

396 We introduced ANNA-PALM, a computational method based on deep learning, that

397 reconstructs high quality super-resolution images from sparse, rapidly acquired, 398 single molecule localization data (and/or widefield images). Our method enables 399 considerable gains in acquisition time compared to standard localization 400 microscopy without increasing active fluorophore density, thereby preserving 401 spatial resolution. In fact, ANNA-PALM even improves spatial resolution when 402 applied to images of lower resolution than the training data 403 (Supplementary Figures 7a, 10), and greatly diminishes the detrimental effect of 404 drift. The improvement in imaging efficiency afforded by ANNA-PALM alleviates the 405 incompatibility between high resolution and high-throughput microscopy by 
406 enabling super-resolution imaging of thousands of cells within a few hours or even

407 less (Figure 4, Supplementary Figure 12). This will facilitate super-resolution

408 studies of rare events, cellular heterogeneity and of partly stochastic structures such

409 as cytoskeletal polymers or chromosomes, whose characterization requires

410 statistics on many configurations ${ }^{38,39}$. ANNA-PALM may also be beneficial for high-

411 throughput imaging screens, e.g. of drug treatments or gene knock-outs ${ }^{40-42}$. In

412 addition, we envision applications to super-resolution imaging of large samples by

413 stitching together multiple images of spatially adjacent fields. The ability to generate

414 images spanning many orders of magnitude in scale could be well adapted to

415 expansion microscopy, a super-resolution technique that physically increases

416 sample size, but often requires tiling many fields of view to image even a single

417 cell ${ }^{43,44}$. With correlative microscopy 45 , it might also be possible to train ANNA-

418 PALM to reconstruct electron microscopy (EM) images from fluorescence images,

419 potentially extending the method to molecular resolutions currently out of reach of

420 localization microscopy. Adaptation of ANNA-PALM to $3 \mathrm{D}^{15,46}$ and multi-color ${ }^{47,48}$

421 localization microscopy should be relatively straightforward. Localization

422 microscopy of cellular dynamics remains very challenging 3,49 . By using much fewer

423 frames (or even only widefield images), ANNA-PALM could dramatically improve

424 the temporal resolution of live cell localization microscopy without sacrificing

425 spatial resolution or increasing phototoxicity and photobleaching. Thus, ANNA-

426 PALM provides multiple novel avenues for multi-scale imaging beyond standard 427 spatio-temporal resolution limits. 
Neverthelesss, important caveats should be stressed. First, although ANNA-

429 PALM can be applied successfully to very different types of images (Figure 6), the

430 method fails in absence of statistical redundancies between molecular localizations,

431 e.g. for entirely random distributions of molecules. Second, ANNA-PALM requires

432 prior training on dense PALM images with structures similar to those in the images

433 to be reconstructed. We showed that ANNA-PALM is robust, i.e. does not require

434 retraining, for some experimentally induced changes in structures and variations in

435 imaging parameters (Figure 5). Nevertheless, indiscriminate application of ANNA-

436 PALM to very different structures without retraining, or incorrect setting of the

437 switch, may result in artifacts (Supplementary Figure 13h). Third, even when

438 applied to data similar to the training images, ANNA-PALM can produce errors -as

439 any reconstruction method in a context of information scarcity. The frequency of

440 errors can be reduced by increasing the number of recorded frames, at the cost of

441 reduced acceleration (see Figures 2,3 and Supplementary Figures 4, 8, 9). In

442 addition, ANNA-PALM can use widefield images to estimate the reliability of

443 reconstructions, thereby helping their interpretation, providing some protection

444 against artifacts and indicating when retraining may be needed (Supplementary

445 Figures 11, 15). Future work, e.g. using Bayesian deep learning50, may provide

446 additional assessments of reconstruction uncertainty and shed more light on the

447 scope and limitations of our approach.

448 As a purely computational technique, ANNA-PALM does not necessitate any

449 changes to existing microscopy systems, but only requires one or a few standard

450 PALM images for training. To facilitate its adoption and future development, we 
451 make our source code, an ImageJ plugin and a cloud-computing based web

452 application available on https://annapalm.pasteur.fr/ along with sample data.

453 Because the performance of deep learning methods improves with the amount and

454 variety of training data, we designed our web application to enable sharing of data

455 and trained ANNs. As ANNA-PALM will learn from an increasing large and diverse

456 collection of images, we expect it to reach even higher accuracy or efficiency and to

457 expand its scope of application in the future.

\section{Acknowledgements}

461 We thank the following colleagues for useful discussions and suggestions and/or

462 critical reading of the manuscript: C. Leduc, S. Etienne-Manneville, S. Lévêque-Fort,

463 N. Bourg, A. Echard, J-B. Masson, T. Rose, P. Hersen, F. Mueller, M. Cohen, Z. Zhang,

464 and P. Kanchanawong. We also thank the four anonymous reviewers for their

465 constructive criticism, which led to significant improvements of ANNA-PALM. We

466 further thank O. Faklaris, J. Sellés and M. Penrad (Institut Jacques Monod), and F.

467 Montel (Ecole Normale Supérieure de Lyon) for providing Xenopus nuclear pore

468 data, B. Jian (Institut Pasteur) for TOM22 antibodies, and C. Leterrier for fixation

469 protocols. We thank E. Rensen and C. Weber for help with experiments and

470 suggestions, B. Lelandais for help with PALM image processing, J-B. Arbona for

471 polymer simulations and J. Parmar for suggestions that led to the name ANNA-

472 PALM. We thank the IT service of Institut Pasteur, including J-B. Denis, N. Joly, and S.

473 Fournier, for access to the HPC cluster and relevant assistance, and T. Huynh for

474 help with GPU computing. This work was funded by Institut Pasteur, Agence 
475 Nationale de la Recherche grant (ANR 14 CE10 0018 02), Fondation pour la 476 Recherche Médicale (Equipe FRM, DEQ 20150331762), and the Région Ile de France 477 (DIM Malinf). We also acknowledge Investissement d'Avenir grant ANR-16-CONV4780005 for funding a GPU farm used in this work. A.A. and X.H. are recipients of 479 Pasteur-Roux fellowships from Institut Pasteur. W.O. is a scholar in the Pasteur 480 Paris University (PPU) International PhD program.

\section{Author contributions}

484 W.O.: conceived method, developed ANNA-PALM software and web application, 485 performed experiments and analyses. A.A., M.L., X.H.: performed experiments. C.Z.: 486 conceived method, supervised project and wrote manuscript.

\section{Competing financial interests statement}

490 W.O. and C.Z. are listed as inventors on European patent application EP17306022 491 filed by Institut Pasteur. 
494

495

496

497

498

499

500

501

502

503

504

505

506

507

508

509

510

511

512

513

514

515

516

517

518

519

520

521

522

523

524

525

526

527

528

529

530

531

532

533

534

535

536

537

538

539

\section{REFERENCES}

1. Betzig, E. et al. Imaging intracellular fluorescent proteins at nanometer resolution. Science (80-. ). 313, 1642-1645 (2006).

2. Rust, M. J., Bates, M. \& Zhuang, X. Sub-diffraction-limit imaging by stochastic optical reconstruction microscopy (STORM). Nat Methods 3, 793-795 (2006).

3. Legant, W. R. et al. High-density three-dimensional localization microscopy across large volumes. Nat. Methods 13, 359-365 (2016).

4. Deschout, H. et al. Precisely and accurately localizing single emitters in fluorescence microscopy. Nat. Methods 11, 253-266 (2014).

5. Jones, S. A., Shim, S. H., He, J. \& Zhuang, X. Fast, three-dimensional superresolution imaging of live cells. Nat Methods 8, 499-505 (2011).

6. Huang, F. et al. Video-rate nanoscopy using sCMOS camera-specific singlemolecule localization algorithms. Nat. Methods 10, 653-8 (2013).

7. Carlton, P. M. et al. Fast live simultaneous multiwavelength four-dimensional optical microscopy. Proc. Natl. Acad. Sci. U. S. A. 107, 16016-22 (2010).

8. Stelzer, E. H. K. Light-sheet fluorescence microscopy for quantitative biology. Nat. Methods 12, 23-26 (2014).

9. Huang, F., Schwartz, S. L., Byars, J. M. \& Lidke, K. A. Simultaneous multipleemitter fitting for single molecule super-resolution imaging. Biomed. Opt. Express 2, 1377-93 (2011).

10. Burnette, D. T., Sengupta, P., Dai, Y., Lippincott-Schwartz, J. \& Kachar, B. Bleaching/blinking assisted localization microscopy for superresolution imaging using standard fluorescent molecules. Proc Natl Acad Sci U S A 108, 21081-21086 (2011).

11. Simonson, P. D., Rothenberg, E. \& Selvin, P. R. Single-molecule-based superresolution images in the presence of multiple fluorophores. Nano Lett 11, 5090-5096 (2011).

12. Zhu, L., Zhang, W., Elnatan, D. \& Huang, B. Faster STORM using compressed sensing. Nat. Methods 9, 721-723 (2012).

13. Cox, S. et al. Bayesian localization microscopy reveals nanoscale podosome dynamics. Nat Methods 9, 195-200 (2012).

14. Ram, S., Ward, E. S. \& Ober, R. J. Beyond Rayleigh's criterion: A resolution measure with application to single-molecule microscopy. Proc. Natl. Acad. Sci. 103, 4457-4462 (2006).

15. Deschout, H. et al. Precisely and accurately localizing single emitters in fluorescence microscopy. Nat. Methods 11, 253-266 (2014).

16. LeCun, Y., Bengio, Y. \& Hinton, G. Deep learning. Nature 521, 436-444 (2015).

17. Michael, E. Sparse and Redundant Representations: From Theory to Applications in Signal and Image Processing. Springer (Springer Verlag, 2010).

18. Hinton, G. E. \& Salakhutdinov, R. R. Reducing the Dimensionality of Data with Neural Networks. Science (80-. ). 313, (2006).

19. Schmidhuber, J. Deep learning in neural networks: An overview. Neural Networks 61, 85-117 (2014).

20. Sage, D. et al. Quantitative evaluation of software packages for single-molecule localization microscopy. Nat. Methods 12, 717-724 (2015). 
540

541

542

543

544

545

546

547

548

549

550

551

552

553

554

555

556

557

558

559

560

561

562

563

564

565

566

567

568

569

570

571

572

573

574

575

576

577

578

579

580

581

582

583

584

585

21. Isola, P., Zhu, J.-Y., Zhou, T. \& Efros, A. A. Image-to-Image Translation with Conditional Adversarial Networks. http://arxiv.org/abs/1611.07004 (2016).

22. Ronneberger, O., Fischer, P. \& Brox, T. U-net: Convolutional networks for biomedical image segmentation. in Medical Image Computing and ComputerAssisted Intervention - MICCAI 2015. Lecture Notes in Computer Science, 9351, 234-241 (Springer International Publishing, 2015).

23. Goodfellow, I. et al. Generative Adversarial Nets. Adv. Neural Inf. Process. Syst. 27 2672-2680 (2014). doi:10.1017/CB09781139058452

24. Ciresan, D., Giusti Alessandro, Luca, G. \& Schmidhuber, J. Mitosis Detection in Breast Cancer Histology Images with Deep Neural Networks. Med. Image Comput. Comput. Interv. - MICCAI 2013 8150, (2013).

25. Wang, Z. W. Z. \& Bovik, A. C. A. C. Mean squared error: Love it or leave it? A new look at Signal Fidelity Measures. IEEE Signal Process. Mag. 26, 98-117 (2009).

26. Zhao, H., Gallo, O., Frosio, I. \& Kautz, J. Loss Functions for Image Restoration with Neural Networks. IEEE Trans. Comput. IMAGING 3, 47-57 (2017).

27. Culley, S. et al. NanoJ-SQUIRREL: quantitative mapping and minimisation of super-resolution optical imaging artefacts. bioRxiv 158279 (2017). doi:10.1101/158279

28. Srivastava, N., Hinton, G., Krizhevsky, A., Sutskever, I. \& Salakhutdinov, R. Dropout: A Simple Way to Prevent Neural Networks from Overfitting. J. Mach. Learn. Res. 15, (2014).

29. Arbona, J.-M., Herbert, S., Fabre, E. \& Zimmer, C. Inferring the physical properties of yeast chromatin through Bayesian analysis of whole nucleus simulations. Genome Biol. 18, 81 (2017).

30. Arnal, I. \& Wade, R. H. How does taxol stabilize microtubules? Curr. Biol. 5, 900-908 (1995).

31. $\mathrm{Wu}, \mathrm{S}$. et al. Microtubule motors regulate ISOC activation necessary to increase endothelial cell permeability. J. Biol. Chem. 282, 34801-8 (2007).

32. De Brabander, M., De May, J., Joniau, M. \& Geuens, G. Ultrastructural immunocytochemical distribution of tubulin in cultured cells treated with microtubule inhibitors. Cell Biol. Int. Rep. 1, 177-83 (1977).

33. Schnitzbauer, J., Strauss, M. T., Schlichthaerle, T., Schueder, F. \& Jungmann, R. Super-resolution microscopy with DNA-PAINT. Nat. Protoc. 12, 1198-1228 (2017).

34. Löschberger, A. et al. Super-resolution imaging visualizes the eightfold symmetry of gp210 proteins around the nuclear pore complex and resolves the central channel with nanometer resolution. J. Cell Sci. 125, 570-5 (2012).

35. Szymborska, A. et al. Nuclear pore scaffold structure analyzed by superresolution microscopy and particle averaging. Science 341, 655-8 (2013).

36. Sellés, J. et al. Nuclear pore complex plasticity during developmental process as revealed by super-resolution microscopy. Sci. Rep. 7, 14732 (2017).

37. Bellot, G. et al. TOM22, a core component of the mitochondria outer membrane protein translocation pore, is a mitochondrial receptor for the proapoptotic protein Bax. Cell Death Differ. 14, 785-794 (2007).

38. Boettiger, A. N. et al. Super-resolution imaging reveals distinct chromatin 
586

587

588

589

590

591

592

593

594

595

596

597

598

599

600

601

602

603

604

605

606

607

608

609

610

611

612

613

614

615

616

617

folding for different epigenetic states. Nature 529, 418-422 (2016).

39. Zhang, Z., Nishimura, Y. \& Kanchanawong, P. Extracting microtubule networks from superresolution single-molecule localization microscopy data. Mol. Biol. Cell 28, 333-345 (2017).

40. Neumann, B. et al. Phenotypic profiling of the human genome by time-lapse microscopy reveals cell division genes. Nature 464, 721-727 (2010).

41. Beghin, A. et al. Localization-based super-resolution imaging meets highcontent screening. Nat. Methods 14, 1184-1190 (2017).

42. Ouyang, W. \& Zimmer, C. The imaging tsunami: Computational opportunities and challenges. Curr. Opin. Syst. Biol. 4, 105-113 (2017).

43. Chen, F., Tillberg, P. W. \& Boyden, E. S. Expansion microscopy. Science (80-. ). 347, 543-548 (2015).

44. Chang, J.-B. et al. Iterative expansion microscopy. Nat. Methods (2017). doi:10.1038/nmeth.4261

45. de Boer, P., Hoogenboom, J. P. \& Giepmans, B. N. G. Correlated light and electron microscopy: ultrastructure lights up! Nat. Methods 12, 503-513 (2015).

46. Huang, B., Wang, W., Bates, M. \& Zhuang, X. Three-dimensional superresolution imaging by stochastic optical reconstruction microscopy. Science (80-. ). 319, 810-813 (2008).

47. Bates, M., Huang, B., Dempsey, G. T. \& Zhuang, X. Multicolor Super-Resolution Imaging with Photo-Switchable Fluorescent Probes. Science (80-. ). 1146598v1 (2007).

48. Jungmann, R. et al. Multiplexed 3D cellular super-resolution imaging with DNA-PAINT and Exchange-PAINT. Nat. Methods 11, 313-318 (2014).

49. Shroff, H., Galbraith, C. G., Galbraith, J. A. \& Betzig, E. Live-cell photoactivated localization microscopy of nanoscale adhesion dynamics. Nat Methods 5, 417423 (2008).

50. Kendall, A. \& Gal, Y. What Uncertainties Do We Need in Bayesian Deep Learning for Computer Vision? Adv. Neural Inf. Process. Syst. 30 (NIPS 2017) pre-proceedings 5580-5590 (2017). 


\section{FIGURE LEGENDS}

\section{FIGURE 1: Overview of ANNA-PALM}

621 ANNA-PALM consists of two main stages: (a) acquisition of training images using 622 standard localization microscopy (PALM) followed by artificial neural network 623 (ANN) training, and (b) reconstruction of super-resolution views and low resolution 624 error maps from new sparse PALM and/or widefield images (inference). 625 (a) Training images are obtained by acquiring one or a few long sequences, of $626 K \sim 10^{3}-10^{5}$ diffraction limited, single molecule image frames, as in standard PALM 627 experiments; optionally, a widefield image $\mathrm{W}$ can also be acquired (top). The 628 acquisition time for each image sequence is $K \Delta t$, where $\Delta t$ is the single frame 629 exposure time. Standard localization microscopy algorithms (grey 'localization' 630 boxes) are used to generate super-resolution images. For each experiment, a highly 631 sampled (dense) super-resolution image (PK) is generated using all (or in practice $632 \geq 95 \%$ ) available $K$ frames. Sparse PALM images (Pk) from the same experiment are 633 obtained by using only $k<<K$ frames. A switch (light blue) can be set to distinguish 634 different types of structures, e.g. nuclear pore complexes ('NPC'), mitochondria 635 ('Mito') or microtubules ('MT'). An ANN (labeled $\mathcal{G}$ for 'generator', orange) is trained 636 by using the sparse PALM images Pk (plus an upsampled version L of the widefield 637 image $\mathrm{W}$ and the switch setting) as inputs and the corresponding dense PALM image 638 PK as target output. During training, the output of the generator $\mathcal{G}$ (Ak) is compared 639 to the target image PK and the widefield image L (if available) via three loss, or error 640 functions (gray bordered boxes): (i) the 'super-resolution reconstruction error' 
641 measures the difference between the reconstructed image Ak and the target PK

642 using a combination of the L1 norm and the MS-SSIM; (ii) the "low resolution

643 reconstruction error' measures the MS-SSIM between the low resolution image WA

644 predicted from the reconstruction Ak and the low resolution image WP predicted

645 from the target image PK. Images WA and WP are predicted using a second ANN,

646 called low-resolution estimator (labeled $Q$, blue) that is trained to produce an

647 approximation of the actual widefield image W based on the MS-SSIM metric; (iii)

648 the 'conditional GAN error' uses a third ANN (labeled $D$ for 'discriminator', red) that

649 attempts to distinguish between real dense PALM images PK and the generator's

650 output Ak. The combined loss functions are iteratively optimized using stochastic

651 gradient descent. (b) A short sequence of diffraction limited single molecule images

652 (with $k<<K$ frames, i.e. acquisition time $k \Delta \mathrm{t}$ ), and an optional widefield image ( $\mathrm{W}^{\prime}$ ),

653 are acquired. Standard localization algorithms generate a sparse (under-sampled)

654 PALM image ( $\mathrm{P}^{\prime} \mathrm{k}$ ). This sparse image P'k (and the upscaled widefield image L' and

655 switch setting) are fed as inputs to the trained generator $\mathcal{G}$, which outputs a

656 reconstructed ANNA-PALM image ( $\left.\mathrm{A}^{\prime} \mathrm{k}\right)$. In addition, the low resolution estimator $Q$

657 predicts a low resolution image WA', which can be compared to the input widefield

658 image W' via the MS-SSIM to produce a low resolution error map (top).

659

660 FIGURE 2: Validation of ANNA-PALM on simulated images

661 (a) Simulated widefield image of microtubules. (b) Simulated sparse PALM image of

662 microtubules with $n=6,834$ localizations. (c) ANNA-PALM reconstruction using only

663 the widefield image a as input. (d) ANNA-PALM reconstruction using both the 
664 widefield image a and the sparse PALM $\mathbf{b}$ image as inputs. (e) Simulated "perfect" 665 PALM image, equivalent to a PALM image with an infinite number of localizations $666(n=\infty)$ and a resolution of $23 \mathrm{~nm}$. This image was used to generate a (by convolution 667 with a Gaussian kernel approximating the microscope point spread function) and $\mathbf{b}$ 668 (by application of Poisson noise). (f) Merged image showing the perfect PALM image 669 e in green and the ANNA-PALM reconstruction $\mathbf{d}$ in red. Note that the ANNA-PALM 670 images c,d provide many high resolution details that are absent from the widefield 671 image a and the sparse PALM image $\mathbf{b}$ and that are in good (c) or very good (d) 672 agreement with the perfect PALM image e. Some reconstruction errors are 673 highlighted by arrows. Blue arrows in panel c point to errors of ANNA-PALM 674 reconstruction from the widefield image only, the white arrow in panel $\mathbf{d}$ points to 675 an error of ANNA-PALM reconstruction from both widefield and sparse PALM 676 images combined. Reconstruction errors diminish for larger numbers of 677 localizations, $n$ (Supplementary Figure 4). (g) Reconstruction quality of PALM and 678 ANNA-PALM images, measured by the MS-SSIM with the perfect PALM image e, as 679 function of localization number $n$. Black curve: reconstruction quality of the 680 standard PALM images. Dashed blue curve: reconstruction quality of ANNA-PALM 681 using the sparse PALM images as input. Solid blue curve: reconstruction quality of 682 ANNA-PALM using both the sparse PALM and widefield images as inputs. Red 683 dashed line: reconstruction quality of ANNA-PALM using the widefield images as 684 input only. Dots are averages from 10 simulations; error bars show standard 685 deviations. The vertical dashed orange line indicates the minimum number of 686 localizations needed to achieve a resolution of $R_{5 \times \mathrm{Nyq}}=23 \mathrm{~nm}$ according to the five- 
687 fold Nyquist criterion ${ }^{3}$. The dashed grey line indicates the minimum number of 688 localizations needed to achieve a double mean nearest neighbor distance less than $68923 \mathrm{~nm}$. ANNA-PALM reconstructions from sparse PALM images only (i.e. without 690 widefield images) achieve the same average MS-SSIM as standard PALM at the five691 fold Nyquist sampling limit with 26 times less localizations (blue double arrow). 692 ANNA-PALM reconstruction quality is highest when using both widefield and sparse 693 PALM images as inputs.

694

695 FIGURE 3: ANNA-PALM imaging of microtubules

696 ANNA-PALM reconstructions of a localization microscopy image of immunostained 697 microtubules. (a) Widefield image. (b) Sparse PALM image obtained from the first $6989 \mathrm{~s}$ of acquisition ( $k=300$ frames, $n=11,740$ localizations). (c) Dense PALM image 699 obtained from a $15 \mathrm{~min}$ long acquisition $(K=30,000$ frames, $N=409,364$ 700 localizations). (d) ANNA-PALM reconstruction from the widefield image a only. 701 (e) ANNA-PALM reconstruction from the sparse PALM image b only. (f) ANNA702 PALM reconstruction from the widefield image a and sparse PLAM image b 703 combined. In panels b-f, pixel values are linearly mapped to colors from the look-up 704 table shown below. Black and white correspond to values $V_{\min }$ and $V_{\max }$ 705 respectively, with $V_{\min }=0$ for all panels, $V_{\max }=3,24,102,102$ and 102 for panels $\mathbf{b}, \mathbf{c}$, 706 d, e and f, respectively. (g-i) Merged images comparing ANNA-PALM 707 reconstructions from panels d-f to the dense PALM image c. ANNA-PALM 708 reconstructions are shown in red, the dense PALM image in green. (j) Gradual 709 improvement of image quality for increasing acquisition time $k \Delta t$, shown for the 
710 area highlighted in the insets of panels a-i. Top row: sparse PALM images. Middle

711 row: ANNA-PALM reconstructions from the sparse PALM images only (without

712 widefield). Bottom row: ANNA-PALM reconstructions from the widefield and sparse

713 PALM images combined. Supplementary Video 1 shows the gradual increase in

714 quality of PALM and ANNA-PALM images with increased acquisition time for the

715 larger region of interest shown in panels a-i.

716

717 FIGURE 4: High-throughput imaging with ANNA-PALM

718 Application of ANNA-PALM to high-throughput imaging of a $1.8 \mathrm{~mm} \times 1.8 \mathrm{~mm}$ area

719 containing more than 1,000 cells. (a) Sparse PALM image of this area, constructed

720 by assembling a mosaic of $33 \times 33=1,089$ sparse PALM images of individual fields of

721 view, obtained from $k=1,000$ raw frames each (with $\Delta \mathrm{t}=10 \mathrm{~ms}$ exposure time per

722 frame, i.e. in $k \Delta t=10 \mathrm{~s}$ ). Total image acquisition time was $1,089 \times 10$ s, i.e. $\sim 3.1$ hours.

723 The sparsity of the image is not apparent at this large scale. (b) ANNA-PALM

724 reconstruction of the image in a, obtained by assembling a mosaic of 1,089

725 individual reconstructions (one per field of view). (c) Magnified view of the green

726 boxed region in a. The inset shows a further magnified view of the yellow boxed

727 region, highlighting the sparsity of the image. (d) Same as c, but for the ANNA-PALM

728 reconstruction. A line profile across a microtubule is shown, with a FWHM $\approx 51 \mathrm{~nm}$.

729 Non-linear contrast adjustment was applied manually for panels $\mathbf{a}$ and $\mathbf{b}$, with black

730 corresponding to values of zero in both panels. In panels $\mathbf{c}$ and $\mathbf{d}$, pixel values were

731 linearly mapped to colors from the look-up table in Figure 3; Black and white

732 correspond to values $V_{\min }$ and $V_{\max }$, respectively, with $\mathrm{V}_{\min }=0$ for all panels, and 
$733 V_{\max }=3$ and 51 for panels $\mathbf{c}$ and $\mathbf{d}$, respectively. See also Supplementary Video 2 for

734 an animated 'zoom-in' highlighting the spatial scales covered by the assembled

735 image. See also Supplementary Figure 12 for ANNA-PALM reconstructions of the

736 same area from the widefield images only.

737

738 FIGURE 5: Robustness of ANNA-PALM to experimental perturbations

739 This figure shows ANNA-PALM reconstructions using an ANN trained on PALM

740 images of microtubules in untreated cells and applied without retraining to sparse

741 localization images of microtubules in different experimental conditions: untreated

742 control cells (a-c); cells treated with $1 \mu \mathrm{M}$ of Taxol (d-f); cells treated with $1 \mu \mathrm{M}$ of

743 Nocodazole (g-i); untreated cells imaged with DNA-PAINT (j-l). (a,d,g,j) Sparse

744 localization images obtained from the first $k$ frames of the acquired image sequence,

745 with $k=500,800,300$, and 400 for $\mathbf{a}, \mathbf{d}, \mathbf{g}$, and $\mathbf{j}$, respectively. (b,e,h,k) ANNA-PALM

746 reconstructions using the sparse localization images immediately to the left as

747 input. (c,f,i,l) Dense localization images obtained from $K=60,000$ frames. Pixel

748 values are linearly mapped to colors from the look-up table in Figure 3. Black and

749 white correspond to values $V_{\min }$ and $V_{\max }$, respectively, with $V_{\min }=0$ for all panels,

750 and $V_{\max }=10,120,90,25,150,40,18,150,50,18,120$, and 200 for panels a, b, c, d,

$751 \mathbf{e}, \mathbf{f}, \mathbf{g}, \mathbf{h}, \mathbf{i}, \mathbf{j}, \mathbf{k}$, and $\mathbf{l}$, respectively.

752

753 FIGURE 6: ANNA-PALM reconstructions of nuclear pores and mitochondria

754 PALM and ANNA-PALM images of nuclear pores (a-c) and mitochondria (d-f).

755 (a) Sparse PALM image of the immunolabeled Xenopus nucleoporin gp210 obtained 
756 from the first $k=3,000$ frames. Note that individual nuclear pores are hard to 757 identify. (b) ANNA-PALM reconstruction of image a. (c) Dense PALM image obtained 758 from all $K=30,000$ frames. (d) Sparse PALM image of the immunolabeled 759 mitochondrial outer membrane protein TOM22, obtained from the first $k=400$ 760 frames. (e) ANNA-PALM reconstruction of image d. (f) Dense PALM image obtained 761 from all $K=30,000$ frames. Pixel values are linearly mapped to colors from the look762 up table shown in Figure 3. Black and white correspond to values $V_{\min }$ and $V_{\max }$ 763 respectively, with $V_{\min }=0$ for all panels, and $V_{\max }=3,51,3,3,128$, and 18 for panels a, 764 b, c, d, e, and f, respectively 


\section{ONLINE METHODS}

\section{Artificial neural network}

768 Our ANN, called 'A-net', is based on the pix2pix architecture21, which is a special

769 conditional generative adversarial network (cGAN) ${ }^{23}$ for image to image

770 "translation", i.e. mapping from one type of image to another. The A-net consists of

771 three distinct neural networks: (i) a generator network $\mathcal{G}$ that produces the

772 reconstructed super-resolution image, (ii) a network $Q$ called 'low resolution

773 estimator' that produces the low resolution error map, (iii) a cGAN discriminator

774 network $D$ that provides the adversarial loss (Figure 1a). The generator network $\mathcal{G}$

775 builds on the U-net architecture, and consists of an encoder-decoder network with

776 skip connections ${ }^{22}$ and 16 convolutional layers. Its inputs and outputs are image

777 patches containing $(256 \mathrm{~m}) \mathrm{x}(256 \mathrm{~m})$ pixels, where $m$ is an integer (we used $m=1$ or 2

778 but this can be adjusted for different sizes of CPU/GPU memory or input images).

779 The input is a sparse PALM image, a widefield image upscaled to the same size (see

780 below), and a computational switch number that allows the network to switch

781 between different types of image structures (e.g. nuclear pores or microtubules).

782 The switch setting is encoded numerically and coupled by convolutional operations

783 into the A-net encoder. The output of the generator $\mathcal{G}$ is a reconstructed image

784 (called ANNA-PALM reconstruction or ANNA-PALM image elsewhere) of the same

785 size as the input images. The low resolution estimator $Q$ has four convolutional

786 layers. It takes the $(256 \mathrm{~m}) \times(256 \mathrm{~m})$ dense PALM image patch or the ANNA-PALM

787 image patch as input and outputs a low resolution image with $(64 \mathrm{~m}) \times(64 \mathrm{~m})$ pixels. 
788 The cGAN discriminator network $D$ has five convolutional layers. Its inputs are three $789(256 \mathrm{~m}) \mathrm{x}(256 \mathrm{~m})$ pixel image patches (the sparse PALM image, the widefield image, 790 and either the ANNA-PALM reconstruction or the corresponding dense PALM 791 image), plus the upscaled widefield image, and its output is a $(30 \mathrm{~m}) \mathrm{x}(30 \mathrm{~m})$ image 792 whose pixel values indicate whether the corresponding input patch is real (i.e. an 793 experimentally obtained conventional PALM image) or produced by the generator $\mathcal{G}$.

794 All convolutional layers are followed by batch normalization ${ }^{51}$. Dropout layers ${ }^{28}$ 795 (with dropout probability $\mathrm{p}=0.5$ ) are introduced in the central layers of the A-net 796 generator and turned on during training, but switched off during inference. 797 Activation functions are rectified linear units (ReLU) $x \rightarrow \sup (x, 0)$ or "leaky" ReLUs $798 x \rightarrow \sup (x, 0)+\inf (\varepsilon x, 0)$ with $\varepsilon=0.2^{52}$, except for the last layer of $\mathcal{G}$, which uses the 799 hyperbolic tangent $x \rightarrow \tanh (x)$ and the last layer of $Q$, which uses a sigmoid 800 function $x \rightarrow(1+\exp (-x))^{-1}$. The A-net architecture is fully described in 801 Supplementary Note 1 and Supplementary Tables 1-4. 802

\section{Training objectives and error map}

804 Each of the three networks mentioned above $(\mathcal{G}, Q$, and $D)$ is associated to a distinct 805 objective function - also called loss- and hereafter noted $\mathscr{L}_{\mathcal{G}}, \mathscr{L}_{Q}$, and $\mathscr{L}_{D}$ respectively. 806 These loss functions are specified in detail below. In the following equations, for 807 notational simplicity, we designate the sparse input image as $\boldsymbol{S}$, the low resolution 808 (widefield) input image as $\boldsymbol{W}$, the corresponding dense PALM image (i.e. the target) 809 as $\boldsymbol{T}$, and the A-net's output as $\boldsymbol{A}=\mathcal{G}(\boldsymbol{S}, \boldsymbol{W})$ (in Figure 1a, images $\boldsymbol{S}, \boldsymbol{T}$ and $\boldsymbol{A}$ are 810 labeled Pk, PK and Ak, respectively); the computational switch indicating the image 
811 type is noted $\boldsymbol{M}$. Low resolution images produced by the low resolution estimator 812 network $Q$ from $\boldsymbol{A}$ and $\boldsymbol{T}$ are designated as $\boldsymbol{W}_{A}=Q(\boldsymbol{A})$ and $\boldsymbol{W}_{T}=Q(\boldsymbol{T})$, 813 respectively.

814 The generator loss function $\mathscr{L}_{\mathcal{G}}$ is the sum of three terms. The first term of $\mathscr{L}_{\mathcal{G}}$ 815 is the super-resolution reconstruction error, hereafter called $\mathscr{L}_{\text {SuperRes }}(\mathcal{G})$. This term 816 penalizes the difference between the generator output $\boldsymbol{A}$ and the target image $\boldsymbol{T}$. 817 Based on a recent analysis of loss functions for image restoration with neural 818 networks ${ }^{26}$, we defined this difference as a weighted average of two quantities: 819 (i) the multi-scale structural similarity index (MS-SSIM) between $\boldsymbol{A}$ and $\boldsymbol{T}$ and (ii) a 820 modification of the L1 norm, where the absolute difference between $\boldsymbol{A}$ and $\boldsymbol{T}$ is 821 smoothed by a Gaussian kernel:

$$
\begin{aligned}
& \mathscr{L}_{\text {SuperRes }}(\mathcal{G})=\mathbb{E}_{(\boldsymbol{S}, \boldsymbol{T}, \boldsymbol{W}, \boldsymbol{M}) \sim p_{\text {data }}(\boldsymbol{S}, \boldsymbol{T}, \boldsymbol{W}, \boldsymbol{M})}\left[\rho\left(1-\operatorname{MS} \_\operatorname{SSIM}(\boldsymbol{A}, \boldsymbol{T})\right)+(1\right. \\
& \left.\quad-\rho)\left\langle G_{\sigma} *|\boldsymbol{A}-\boldsymbol{T}|\right\rangle\right] \\
& \quad=\mathbb{E}_{(\boldsymbol{S}, \boldsymbol{T}, \boldsymbol{W}, \boldsymbol{M}) \sim p_{\text {data }}(\boldsymbol{S}, \boldsymbol{T}, \boldsymbol{W}, \boldsymbol{M})}\left[\rho\left(1-\operatorname{MS} \operatorname{SSIM}_{(\mathcal{G}}(\boldsymbol{S}, \boldsymbol{W}, \boldsymbol{M}), \boldsymbol{T}\right)\right)+(1 \\
& \left.\quad-\rho)\left\langle G_{\sigma} *|\mathcal{G}(\boldsymbol{S}, \boldsymbol{W}, \boldsymbol{M})-\boldsymbol{T}|\right\rangle\right]
\end{aligned}
$$

822 where $\mathbb{E}$ denotes expectation; $p_{\mathrm{data}}(\boldsymbol{S}, \boldsymbol{T}, \boldsymbol{W}, \boldsymbol{M})$ is the joint probability density of the 823 sparse PALM images $\boldsymbol{S}$, dense PALM images $\boldsymbol{T}$, widefield images $\boldsymbol{W}$ and switch 824 settings $\boldsymbol{M}$ from the training data set; $\operatorname{MS} \operatorname{SSIM}(\boldsymbol{A}, \boldsymbol{T})$ is the multi-scale structural 825 similarity index between $\boldsymbol{A}$ and $\boldsymbol{T} ; G_{\sigma}$ is a Gaussian smoothing kernel; * denotes 826 convolution; $|\boldsymbol{A}-\boldsymbol{T}|$ is the absolute difference image (i.e. pixel (i,j) has value $827|\boldsymbol{A}(\mathrm{i}, \mathrm{j})-\boldsymbol{T}(\mathrm{i}, \mathrm{j})|$ and $\rho \in[0,1]$ is a scalar weight that balances the relative 828 contributions of MS-SSIM and the modified L1 norm and is set to $\rho=0.84$ as in ref. 82926. 
831 between the low resolution images $\boldsymbol{W}_{A}$ and $\boldsymbol{W}_{T}$ predicted by the low resolution 832 estimator network $Q$ :

$$
\begin{aligned}
\mathscr{L}_{\text {LowRes }}(\mathcal{G}, Q) & =\mathbb{E}_{(\boldsymbol{S}, \boldsymbol{T}, \boldsymbol{W}, \boldsymbol{M}) \sim p_{\mathrm{data}}(\boldsymbol{S}, \boldsymbol{T}, \boldsymbol{W}, \boldsymbol{M})}\left[1-\operatorname{MS} \operatorname{SSIM}_{-}\left(\boldsymbol{W}_{A}, \boldsymbol{W}_{T}\right)\right] \\
& =\mathbb{E}_{(\boldsymbol{S}, \boldsymbol{T}, \boldsymbol{W}, \boldsymbol{M}) \sim p_{\text {data }}(\boldsymbol{S}, \boldsymbol{T}, \boldsymbol{W}, \boldsymbol{M})}\left[1-\operatorname{MS} \operatorname{SSIM}_{-}(Q(\mathcal{G}(\boldsymbol{S}, \boldsymbol{W}, \boldsymbol{M})), Q(\boldsymbol{T}))\right]
\end{aligned}
$$

833 Alternatively, in the above objective function, $\boldsymbol{W}_{T}$ can be replaced by the actually 834 observed widefield image $\boldsymbol{W}$, although with our data this led to slightly lower 835 reconstruction quality. The low resolution estimator network $Q$ is trained 836 simultaneously with the generator $\mathcal{G}$ to produce a low resolution image from the 837 dense PALM image $\boldsymbol{T}$ that is consistent with the observed low resolution image $\boldsymbol{W}$.

838 This training is done based on the following objective function:

$$
\mathscr{L}_{Q}(Q)=\mathbb{E}_{(\boldsymbol{T}, \boldsymbol{W}) \sim p_{\text {data }}(\boldsymbol{T}, \boldsymbol{W})}\left[1-M S_{-} \operatorname{SSIM}(Q(\boldsymbol{T}), \boldsymbol{W})\right]
$$

839 Note that the reconstructed low resolution image $Q(\boldsymbol{T})$ is four times smaller than 840 the dense PALM image $\boldsymbol{T}$, as described in Supplementary Note 1. Because the input 841 widefield image $\boldsymbol{W}$ can have a different size, we use bilinear interpolation to resize $842 W$ to the same size as $Q(\boldsymbol{T})$. (If needed, a scaling factor different from four can be 843 obtained by adding or removing downsample layers in network $Q$ ). At inference, the 844 low resolution estimator $Q$ is also used to produce the error map, as shown in 845 Figure 1b, and Supplementary Figures 6, 11, 15. This error map is defined as:

$$
\boldsymbol{E}_{Q}(\boldsymbol{A}, \boldsymbol{W})=\left(1-M S_{-} \operatorname{SSIM}(Q(\boldsymbol{A}), \boldsymbol{W})\right)(Q(\boldsymbol{A})+\boldsymbol{W})
$$

846 High (respectively low) values of the error map indicate large (respectively small) 847 inconsistencies, between the reconstructed super-resolution image $\boldsymbol{A}$ and the 848 observed widefield image $\boldsymbol{W}$. 
850 networks $(\mathrm{GAN})^{21,23,53}$ and is noted $\mathscr{L}_{\mathrm{cGAN}}(\mathcal{G}, \mathcal{D})$. In a GAN, a generator network $\mathcal{G}$

851 learns to transform random input vectors $\mathbf{z}$ (drawn from a probability density $852 p_{\mathrm{z}}(\mathbf{z})$ ) into new samples of a data probability density $p_{\text {data }}(\boldsymbol{x})$. In our case, the data 853 samples $\boldsymbol{x}$ are the dense PALM images $\boldsymbol{T}$. The generator $\mathcal{G}$ learns by working against 854 a discriminator network $\mathcal{D}$ that simultaneously learns to discriminate between 855 original data samples and samples generated by $\mathcal{G}$. Adversarial training thus 856 consists in playing a minmax game such that $\left(\mathcal{G}^{*}, \mathcal{D}^{*}\right)=\arg \min _{\mathcal{G}} \max _{\mathcal{D}} \mathcal{L}_{\mathrm{GAN}}(\mathcal{G}, \mathcal{D})$, 857 with an objective function of the form ${ }^{23}: \mathcal{L}_{\mathrm{GAN}}(\mathcal{G}, \mathcal{D})=\mathbb{E}_{\boldsymbol{x} \sim p_{\text {data }}(\boldsymbol{x})}[\log \mathcal{D}(\boldsymbol{x})]+$ $858 \mathbb{E}_{\mathbf{z} \sim p_{\mathbf{Z}}(\mathbf{z})} \log [1-\mathcal{D}(\mathcal{G}(\mathbf{z}))]$, or equivalently by simultaneous optimization of two 859 coupled loss functions:

$$
\left\{\begin{array}{c}
\mathcal{D}^{*}=\arg \max _{\mathcal{D}}\left(\mathbb{E}_{\boldsymbol{x} \sim p_{\text {data }}(\boldsymbol{x})}[\log \mathcal{D}(\boldsymbol{x})]+\mathbb{E}_{\mathbf{z} \sim p_{\mathbf{Z}}(\mathbf{z})} \log [1-\mathcal{D}(\mathcal{G}(\mathbf{z}))]\right) \\
\mathcal{G}^{*}=\arg \min _{G}\left(\mathbb{E}_{\mathbf{z} \sim p_{\mathbf{Z}}(\mathbf{z})} \log [1-\mathcal{D}(\mathcal{G}(\mathbf{z}))]\right)
\end{array}\right.
$$

860 In a conditional GAN ( $\mathrm{CGAN}$ ), the generator and the discriminator have an extra 861 input vector $\boldsymbol{c}$ and the first objective function above becomes: $\mathcal{L}_{\mathcal{D}}(\mathcal{G}, \mathcal{D})=$ $862 \mathbb{E}_{(\boldsymbol{c}, \boldsymbol{x}) \sim p_{\text {data }}(\boldsymbol{c}, \boldsymbol{x})}[\log \mathcal{D}(\boldsymbol{c}, \boldsymbol{x})]+\mathbb{E}_{\boldsymbol{c} \sim p_{\text {data }}(\boldsymbol{c}), \mathbf{z} \sim p_{\mathrm{Z}}(\mathbf{z})} \log [1-\mathcal{D}(\boldsymbol{c}, \mathcal{G}(\boldsymbol{c}, \boldsymbol{z}))]$, such that the 863 generator learns a conditional probability density $p_{\text {data }}(\boldsymbol{x} \mid \boldsymbol{c})$; and the second 864 objective function likewise becomes $\mathcal{L}_{\mathrm{cGAN}}(\mathcal{G}, \mathcal{D})=\mathbb{E}_{\boldsymbol{c} \sim p_{\text {data }}(c), \boldsymbol{z} \sim p_{\mathrm{z}}(\mathbf{z})} \log [1-$ $865 \mathcal{D}(\mathcal{G}(\mathbf{z}))]$. In our A-net, we replaced the logarithmic losses above by least square $866 \operatorname{losses}^{53}$, as they empirically yielded better results. Thus, we used the objective 867 functions:

$868 \mathcal{L}_{\mathcal{D}}(\mathcal{G}, \mathcal{D})=\mathbb{E}_{(\boldsymbol{c}, \boldsymbol{x}) \sim p_{\text {data }}(\boldsymbol{c}, \boldsymbol{x})}(\mathcal{D}(\boldsymbol{c}, \boldsymbol{x})-1)^{2}+\mathbb{E}_{\boldsymbol{c} \sim p_{\text {data }}(\boldsymbol{c}), \mathbf{z} \sim p_{\mathrm{Z}}(\mathbf{z})}[\mathcal{D}(\boldsymbol{c}, \mathcal{G}(\boldsymbol{c}, \boldsymbol{z}))]^{2} \quad$ and 
$869 \mathcal{L}_{\mathrm{cGAN}}(\mathcal{G}, \mathcal{D})=\mathbb{E}_{\boldsymbol{c} \sim p_{\text {data }}(\boldsymbol{c}), \mathbf{z} \sim p_{\mathrm{Z}}(\mathbf{z})}[1-\mathcal{D}(\boldsymbol{c}, \mathcal{G}(\boldsymbol{c}, \mathbf{z}))]^{2}$. In our case, the input $\boldsymbol{c}$ is the 870 sparse PALM image $\boldsymbol{S}$ combined with the upsampled version $\boldsymbol{L}=\mathcal{B}(\boldsymbol{W})$ of the 871 widefield image $\boldsymbol{W}$, where $\mathcal{B}$ denotes bilinear interpolation. Note that in practice the 872 noise $\mathbf{z}$ in our ANN was introduced only through the use of dropout layers, as in the 873 pix2pix implementation ${ }^{21}$. Thus, the objective functions are:

$$
\begin{aligned}
\mathscr{L}_{\mathcal{D}}(\mathcal{D})=\mathbb{E}_{\boldsymbol{S}, \boldsymbol{T}, \boldsymbol{W}, \boldsymbol{M} \sim p_{\text {data }}(\boldsymbol{S}, \boldsymbol{T}, \boldsymbol{W}, \boldsymbol{M})}(\mathcal{D}(\mathcal{B}(\boldsymbol{W}), \boldsymbol{S}, \boldsymbol{T})-1)^{2} \\
\quad+\mathbb{E}_{\boldsymbol{Z} \sim p_{\mathrm{Z}}(\boldsymbol{z}), \boldsymbol{S} \sim p_{\text {data }}(\boldsymbol{S})}(\mathcal{D}(\mathcal{B}(\boldsymbol{W}), \boldsymbol{S}, \mathcal{G}(\boldsymbol{S}, \boldsymbol{W}, \boldsymbol{M})))^{2}
\end{aligned}
$$

874 and:

$$
\mathscr{L}_{\mathrm{CGAN}}(\mathcal{G}, \mathcal{D})=\mathbb{E}_{(\boldsymbol{S}, \boldsymbol{T}, \boldsymbol{W}, \boldsymbol{M}) \sim p_{\mathrm{data}}(S, T, W, M)}(\mathcal{D}(\mathcal{B}(\boldsymbol{W}), \boldsymbol{S}, \mathcal{G}(\boldsymbol{S}, \boldsymbol{W}, \boldsymbol{M}))-1)^{2}
$$

875

In the end, combining the three loss terms described above, we implemented 876 the following optimization problem :

$$
\left\{\begin{array}{c}
Q^{*}=\arg \min _{Q} \mathscr{L}_{Q}(Q) \\
\mathcal{D}^{*}=\arg \max _{\mathcal{D}} \mathscr{L}_{\mathcal{D}}(\mathcal{D}) \\
\mathcal{G}^{*}=\arg \min _{\mathcal{G}}\left[\alpha \mathscr{L}_{\text {SuperRes }}(\mathcal{G})+\beta \mathscr{L}_{\text {LowRes }}(\mathcal{G}, Q)+\gamma \mathscr{L}_{\text {CGAN }}(\mathcal{G}, \mathcal{D})\right]
\end{array}\right.
$$

877 The weights $\alpha, \beta$ and $\gamma$ are hyperparameters, which we set manually to $\alpha=50$, $878 \beta=25$ and $\gamma=1$ for most experiments. In absence of widefield images $\boldsymbol{W}, \beta$ was 879 simply set to zero. The reported results are not very sensitive to these parameters.

880 We trained the A-net end-to-end using stochastic gradient descent (SGD) 881 with Adam $^{54}$ and a batch size of 1 with 200,000 or more iterations (backpropagation 882 steps). Our implementation was adapted from affinelayer's TensorFlow 55 883 implementation, which is ported from the Torch implementation of pix2pix ${ }^{21}$. Both 884 network training and inference were performed on Tesla P100, Tesla M40, Tesla 
885 K80 or GTX TitanXP graphical processing units (GPUs) from Nvidia. A-net training 886 from scratch typically takes from hours to days on a single GPU. Once trained, the A887 net takes only $\sim 1$ second or less to reconstruct a super-resolution image of $8882560 \times 2560$ pixels (corresponding to an entire FoV). Training time could be further 889 reduced by pretraining (or transfer learning), use of GPU clusters, or optimized data 890 augmentation.

891

892 Experimental training images and data augmentation

893 Experimental training data are obtained from standard localization microscopy data 894 (dense PALM images). To achieve good performance, ANNs generally necessitate 895 large amounts of training data. However, ANNA-PALM typically requires PALM 896 images from no more than 10 FoVs (of $55 \mu \mathrm{m} \times 55 \mu \mathrm{m}$ each) and can even be trained 897 with a single FoV. This is possible thanks to an extensive on-the-fly data 898 augmentation strategy, as described below. Each of the dense PALM training images 899 corresponds to a list of localizations $\left(f_{i}, x_{i}, y_{i}\right)_{i=1 . n}$, where $f_{i} \in[1, K]$ is the index of 900 the diffraction limited frame from which localization $x_{i}, y_{i}$ originates, and $K$ is the 901 total frame number. PALM images are obtained as plain 2D histograms of these 902 localizations with typical pixel sizes of $10-20 \mathrm{~nm}$. From each list of localizations 903 (corresponding to a dense PALM image of a single FoV), we generate 10-30 pairs of 904 input and target images $(\boldsymbol{S}, \boldsymbol{T})$ for training. To define the target image $\boldsymbol{T}$, we take a 905 random consecutive subset $\left[k_{T}, k_{T}+0.95 K\right]$ of $95 \%$ of all available $K$ frames $\left(k_{T}\right.$ is 906 chosen randomly between 0 and $0.05 K$ ) and create the 2D histogram image based 907 on localizations from those frames only, i.e. from all $\left(x_{j}, y_{j}\right)$ such that $f_{j} \in$ 
$908\left[k_{T}, k_{T}+0.95 K\right]$. To define the sparse input image $\boldsymbol{S}$, we take random subsets of $909300-500$ consecutive frames from the first half of the image sequence and similarly 910 create a 2D histogram of the localizations from those frames only. When a widefield 911 image $\boldsymbol{W}$ is available, this image must first be aligned with the corresponding dense

912 PALM image $\boldsymbol{T}$. This is done using an FFT-based phase correlation algorithm ${ }^{56}$ after 913 histogram equalization of image $\boldsymbol{T}$, smoothing by convolution with a Gaussian

914 Kernel of standard deviation 6 pixels, and resizing with bilinear interpolation to the 915 same size as image $\boldsymbol{W}$. The registered widefield image $\boldsymbol{W}$ is then scaled up using 916 bilinear interpolation to an image $\boldsymbol{L}=\mathcal{B}(\boldsymbol{W})$ with the same size as image $\boldsymbol{T}$.

917 During training, for each iteration of SGD, we crop the images $\boldsymbol{S}, \boldsymbol{L}$ and $\boldsymbol{T}$ with 918 a randomly placed $712 \times 712$ pixel sized region $\mathscr{R}=\left[x_{\min }, x_{\min }+712\right] \times$ $919\left[y_{\min }, y_{\min }+712\right]$. We then use random geometric transformations and apply them 920 identically to the three images. Specifically, we rotate the images by a random angle 921 between 0 and 360 degrees, apply elastic transformations ${ }^{57}$, and then crop the 922 center region of size $512 \times 512$ pixels. In addition to geometric transformations, we 923 also introduce realistic noise from experimental background images. This is done by 924 manually outlining regions of background in selected PALM training images, 925 splitting these regions into small patches of 40x40 pixels, grouping them according 926 to their summed pixel values, then assembling them into a larger image with the 927 same size as $\boldsymbol{T}$. During training, these semi-synthetic noise images are randomly 928 selected and added to the input image $\boldsymbol{S}$ without altering $\boldsymbol{T}$ or $\boldsymbol{W}$. Finally, we 929 normalize the input image $\boldsymbol{S}$ by subtracting its mean and dividing by standard 930 deviation. If a widefield image $\boldsymbol{W}$ is provided, its pixel values are scaled to a 
931 minimum of 0 and a maximum of 1 . Otherwise, $\boldsymbol{W}$ is replaced by an image containing

932 zeros only. The target image $\boldsymbol{T}$ is truncated at a maximum value of 255 and then

933 scaled to have a minimum of 0 and a maximum of 1 . For the switch $\boldsymbol{M}$, we used an

934 integer number to define the type of training images, e.g. 0 for microtubules, 1 for

935 nucleoporins, 2 for mitochondria. When training on different types of images, e.g.

936 microtubules and nucleoporins (Supplementary Figure 13), we assign the

937 corresponding switch value to $\boldsymbol{M}$ and use it as additional input to the A-net together

938 with images $\boldsymbol{S}$ and $\boldsymbol{W}$, as described in Supplementary Note 1.

939

940 Image simulations

941 Our procedure to simulate localization microscopy (PALM) images of microtubules

942 is illustrated in Supplementary Figure 1. To simulate microtubule filaments, we

943 used a Langevin dynamics simulation ${ }^{29}$ that generates random configurations of

944 semiflexible curves with a specified rigidity (persistence length), starting from a

945 random initial configuration (Supplementary Figure 1a,b). The initial

946 configurations were generated with a Python library named cpolymer and the

947 Langevin dynamics was implemented using the molecular dynamics code

948 LAMMPS ${ }^{58}$. Although the simulation generates 3D polymer chains, we only

949 considered their 2D projections, consisting of $N_{p}$ connected positions $\left(x_{k}, y_{k}\right)_{k=1 . . N_{p}}$.

950 To obtain smooth filaments we further interpolated these connected segments using

951 spline functions with the Scipy function scipy.interpolate.splev. Next, we turned

952 these 2D curves into a grey scale image of $800 x 800$ pixels, with an assumed pixel

953 size of $7.8 \mathrm{~nm}$, using the Python library Matplotlib. This image was further 
954 convolved with a Gaussian kernel of standard deviation 1.5 pixels, resulting in a 955 smooth image $I(i, j)$ as shown in Supplementary Figure 1c, and normalized to a 956 probability density $\left(\sum \sum_{i, j} I(i, j)=1\right.$, with all $\left.I(i, j) \geq 0\right)$. This image was used to 957 mimic a "perfect" PALM image of filaments corresponding to an infinite number of 958 localizations $(n=\infty)$. Such perfect images were used as targets during ANN 959 training for simulated data and defined as ground truth for the quantification of 960 reconstruction quality by MS-SSIM (Figure 2g). During training, we applied the 961 same rotations and elastic transformations described for experimental data in the 962 previous section.

963 Localization microscopy images obtained from a finite number of 964 localizations $n<\infty$ (sparse PALM images $\boldsymbol{S}$ ), can be considered as a sampling of the 965 probability density $I(i, j)$ with $n$ samples. These images can therefore be simulated 966 by applying Poisson noise to a rescaled version of the perfect PALM image, i.e.: $967 \boldsymbol{S}(\lambda, I)=\mathcal{P}\left(\lambda I / I_{\max }\right)$, where $I_{\max }$ is the maximum value of $I, \mathcal{P}(\mu)$ denotes the 968 Poisson probability distribution of mean $\mu$ and where the peak parameter $\lambda$ controls 969 the level of sampling. In order to simulate sparse PALM images for various levels of 970 sampling, we varied the peak value $\lambda$ following a log-normal distribution where $971 \ln (\lambda)$ has mean -0.5 and standard deviation 0.001 and applied Poisson noise using 972 the numpy library function random.poisson. An example of a simulated sparse 973 PALM image is shown in Supplementary Figure 1d. Besides finite sampling, 974 localization microscopy images are corrupted by additional noise sources such as 975 false detections from background noise due to out-of-focus light or unspecific 976 binding of antibodies. To mimic this, we first created a probability density 
$977 I_{b}=I * G_{\sigma_{b}}$ for the background noise by convolving $I$ with a Gaussian kernel $G_{\sigma_{b}}$ of 978 large standard deviation $\sigma_{b}=25$ pixels, and applied Poisson noise with $\lambda=0.06$. To 979 create training images, we added this background noise image to the sparse PALM 980 image $\boldsymbol{S}$ above (Supplementary Figure 2). We did not add background noise to the 981 test images used during inference (Figure 2). To simulate the widefield images 982 (Figure 2a), we first blurred the perfect PALM image by convolution with a 983 Gaussian kernel of standard deviation 8 pixels, then added Gaussian noise with zero 984 mean and standard deviation chosen randomly between 0.5 and 1.5 .

985 For simulations of nuclear pore images (Supplementary Figure 15), we 986 applied a similar procedure, except that the perfect PALM images were obtained by 987 randomly distributing circles of diameter $150 \mathrm{~nm}$ in the plane (avoiding overlaps) 988 and placing eight Gaussian spots (of standard deviation 1.7 pixels) at equal distance 989 from each other on each circle to mimic the octogonal shape of nuclear pores. 990

\section{Sample preparation}

992 For microtubule imaging experiments (Figures 3-5 and Supplementary Figures 8993 10), except those using DNA-PAINT (Figure 5j,I), U-373 MG (Uppsala) cells were 994 cultured in Dulbecco's Modified Eagle Medium: Nutrient Mixture F-12 (DMEM/F12; 995 Gibco) supplemented with 10\% (v/v) fetal bovine serum (FBS; Gibco), 1\% (v/v) 996 penicillin-streptomycin (Gibco), in a $5 \% \mathrm{CO}_{2}$ environment at $37^{\circ} \mathrm{C}$ on $18-\mathrm{mm}$ 997 cleaned coverslips in 12-well plates. 24 hours after plating, cells were pre-extracted 998 for $10 \mathrm{~s}$ in $0.25 \%$ (v/v) Triton X-100 (Triton) in BRB80 (80 mM PIPES, $1 \mathrm{mM} \mathrm{MgCl2}$, 9991 mM EGTA, adjusted to pH 6.8 with $\mathrm{KOH}$ ) supplemented with 4 mM EGTA, and 
1000 immediately fixed for 10 min with $0.25 \%(\mathrm{v} / \mathrm{v})$ Triton $+0.5 \%$ Glutaraldehyde in 1001 BRB80, followed by reduction for 7 min with $0.1 \%$ NaBH4 solution in PBS and 1002 another washing step in PBS. Cells were directly incubated for $1 \mathrm{~h}$ at room 1003 temperature in PBS with 1:500 rat alpha-tubulin antibodies (Bio-Rad MCA77G), 1004 followed by 3 washing steps with PBS, and then incubated for 45min in PBS with 1005 1:500 anti-rat Alexa-647 conjugated secondary antibodies from donkey (Jackson 1006 ImmunoResearch Laboratories, ref. 712-605-153).

1007 For the DNA-PAINT experiment on microtubules (Figure 5k-m), U-373 cells 1008 stuck on $18 \mathrm{~mm}$ diameter coverslips were fixed at $37^{\circ} \mathrm{C}$ with $4 \%$ PFA in PHEM 1009 buffer and permeabilized in $0.2 \%$ glutaldehyde. Next, cells were incubated for $1 \mathrm{~h}$ 1010 with 1:500 primary mouse antibodies against alpha-tubulin. The sample was 1011 washed 3 times in PBS, then incubated with 1:100 anti-mouse oligo-conjugated 1012 antibodies from Ultivue Kit 2 for DNA-PAINT imaging 33 . After washing the sample 3 1013 times in PBS, and just before imaging, 2nM of complementary oligos coupled to Cy3 1014 fluorophores were added to the sample.

1015 Nuclear pore imaging data of gp210 and WGA (Figure 6a-c and 1016 Supplementary Figure 14) were kindly provided by J. Sellés and O. Falklaris and 1017 obtained from nuclear membranes of Xenopus frog eggs prepared as described 1018 previously 36.

1019 For mitochondria imaging experiments (Figure 6e-f), COS7 cells were 1020 cultured under the same conditions as U-373 cells above using phenol-red free 1021 DMEM medium and fixed with 4\% PFA in PBS for 10 min. The sample was blocked 1022 with 3\% BSA in PBS for 20 min and immunostained with 1:500 mouse antibodies 
1023 against TOM22 (Sigma, ref. T6319) in wash buffer (PBS with 0.5\% BSA) for $1 \mathrm{~h}$.

1024 After extensive washing with wash buffer, the sample was incubated with 1:500 1025 anti-mouse secondary antibodies from donkey conjugated to Alexa-647 dyes 1026 (Jackson ImmunoResearch Laboratories, ref. 715-605-151) in wash buffer for 30 1027 min. After washing 5 times with wash buffer and 2 times with PBS, samples were 1028 post-fixed with 2\% PFA in PBS for 10 min and washed 5 times with PBS.

1029 For all localization microscopy experiments except DNA-PAINT, we used a 1030 photoswitching buffer ${ }^{59}$ composed of $50 \mathrm{mM}$ Tris- $\mathrm{HCl}+10 \mathrm{mM} \mathrm{NaCl}+10 \%(\mathrm{w} / \mathrm{v})$ 1031 glucose + 168 AU/mL Glucose-Oxidase + 1404 AU/mL Catalase + 1\% 21032 Mercaptoethanol. For microtubule imaging experiments, we used this buffer to fill a 1033 square hole that was manually cut in a parafilm sheet, which was deposited on a 1034 rectangular coverglass. The round coverslips were sealed with nail polish.

1035

1036 Image acquisition in localization and high-throughput microscopy

1037 We performed single molecule localization microscopy experiments (PALM/STORM 1038 and DNA-PAINT) on custom built microscopy systems, as previously described ${ }^{59-61 .}$

1039 The system used for PALM/STORM imaging of microtubules is based on an inverted 1040 microscope body (Nikon Ti Eclipse) equipped with a either a 60x $1.49 \mathrm{NA}$ oil 1041 immersion objective (Nikon) or a 60x 1.2 NA water immersion objective (Nikon) 1042 and with the Perfect Focus System active. A $642 \mathrm{~nm}$ wavelength laser with $500 \mathrm{~mW}$ 1043 power was used to excite Alexa-647 fluorophores and an AOTF (AA optics) was used 1044 to modulate laser excitation. Sequences of diffraction limited single molecule image 1045 frames were acquired either on a sCMOS camera (Hamamatsu ORCA-Flash4.0), 
1046 which can capture images of 2,042x2,042 pixels (for Figures 4, 5a-i), or on an 1047 EMCCD (Andor IXON ULTRA 897) with 512x512 pixels (for Figures 3, 6d-f). Both 1048 cameras were controlled by MicroManager software ${ }^{62}$. For experiments using the 1049 sCMOS camera, the effective pixel size was $108 \mathrm{~nm}$ and we used a $512 \times 512$ region of 1050 interest, which resulted in an imaged FoV of $55.3 \mu \mathrm{m} \times 55.3 \mu \mathrm{m}$. For experiments 1051 using the EMCCD camera, we used a 2x telescope and the effective pixel size was $1052107 \mathrm{~nm}$, resulting in a FoV of $54.8 \mu \mathrm{m} \times 54.8 \mu \mathrm{m}$. The exposure time was set to $\Delta \mathrm{t}$ $1053=10 \mathrm{~ms}$ or $30 \mathrm{~ms}$ per frame. The number of frames acquired ranged from $k=1,000$ 1054 (Figure 4) to $K=60,000$ (e.g. Figure 5c) per FoV.

1055 For the DNA-PAINT experiment (Figure 5j,l), we used an inverted Nikon Ti-E 1056 Eclipse microscope equipped with a 100x 1.49 NA TIRF objective and with the 1057 Perfect Focus System active. A $561 \mathrm{~nm}$ wavelength laser with $500 \mathrm{~mW}$ power was 1058 used to excite Cy3 dyes. Highly inclined laser illumination was used to reduce out1059 of-focus background signal. Images were acquired on an EMCCD camera as above, 1060 with a $1.5 \mathrm{x}$ telescope, resulting in an effective pixel size of $106 \mathrm{~nm}$ and a FoV of $106154 \mu \mathrm{m} \times 54 \mu \mathrm{m}$. The sample was mounted in a magnetic sample holder filled with 1062 the imaging buffer provided with the Ultivue kit. Exposure time was set to $\Delta \mathrm{t}=30 \mathrm{~ms}$ 1063 and the EM gain of the EMCCD was set to 300. The laser power was increased until 1064 isolated fluorescent spots were observed. For the experiment shown in Figure 5g,i, $1065 K=60,000$ frames were acquired.

1066 The Xenopus nuclear pore data (Figure 6a-c and Supplementary 1067 Figure 14), were acquired on a Zeiss Elyra P.S.1 microscope as described 1068 previously 36. 
1070 Dimensional Acquisition tool in Micro-manager to define the positions of 1,089 FoVs 1071 of $55.3 \mu \mathrm{m} \times 55.3 \mu \mathrm{m}$ on a $33 \times 33$ grid, with overlaps of $1 \mu \mathrm{m}$; the stage was 1072 automatically shifted to each of these 1,089 positions. We first acquired only 1073 widefield images, taking five frames at each of these positions (the first two were 1074 ignored because of motion blur), in a total acquisition time of 12 minutes. Then, the 1075 laser power was raised to bleach out preactivated molecules and $k=1,000$ frames of 1076 single molecule images were acquired at each of the 1,089 positions, in a total 1077 acquisition time of 3 hours and 8 minutes. Raw image frames were written directly 1078 to a remote storage server via Samba networking protocol.

1080 Localization microscopy image analysis

1081 The input to ANNA-PALM reconstruction is a localization image, defined as a 2D 1082 histogram of $n$ single molecule positions $\left(x_{i}, y_{i}\right)_{i=1 . . n}$. The histogram bin, i.e. the 1083 pixel size of the localization image, was set to $7.8 \mathrm{~nm}$ for the simulated data 1084 (Figure 2 and Supplementary Figures 1-6,15) and $20 \mathrm{~nm}$ for the experimental 1085 data (Figures 3-6 and Supplementary Figures 7-14). The positions $\left(x_{i}, y_{i}\right)_{i=1 . . n}$ 1086 were obtained by analyzing sequences of diffraction limited frames using standard 1087 single molecule localization algorithms. For experimental microtubule images, we 1088 used the ThunderSTORM ${ }^{63}$ plugin of ImageJ, applying wavelet filters for detection 1089 and weighted least squares Gaussian fitting for precise estimation of subpixelic 1090 positions. We used the cross-correlation feature in ThunderSTORM for drift 1091 correction, and filtered out the least certain localizations based on the fitted 
1092 Gaussian's standard deviation and the $\chi^{2}$ of the residual. Localizations in 1093 consecutive frames separated by less than $20 \mathrm{~nm}$ were assumed to originate from 1094 the same molecule and merged into a single localization. The final number $n$ of 1095 localizations was $\sim 7$ million for the full $55 \mu \mathrm{m}$ x $55 \mu \mathrm{m}$ FoV of the images shown in 1096 Figure 3 and Supplementary Figures 8,9 (obtained from $K=30,000$ frames). For 1097 the high-throughput experiment (Figure 4 and Supplementary Figure 12), the 1098 number of localizations per $55 \mu \mathrm{m} \times 55 \mu \mathrm{m}$ FoV ranged from $n=2,949$ to $1099 n=1,442,048$ with an average $\langle n\rangle=610,983$ and standard deviation $\sigma(n)=273,606$.

1100 The total number of localizations across all 1,089 FoVs was $\approx 665$ million.

1101 ThunderSTORM analyses were performed either on high end workstations or on 1102 Institut Pasteur's high performance computer (HPC) cluster. For the high1103 throughput experiments, we used Python scripts to run ThunderSTORM in batch 1104 mode (without user intervention) on the HPC cluster and assembled mosaic images 1105 (Figure 4a,b and Supplementary Figure 12a,b) using a stitching plugin of 1106 Image $^{64}$.

1107 The nuclear pore images were analyzed using the ZEN software from Zeiss as 1108 previously described ${ }^{36}$. For the DNA-PAINT experiments, we used PALMTT, a 1109 modified version of the single molecule tracking algorithm MTT65, based on Matlab 1110 (Mathworks). This algorithm uses Gaussian smoothing and thresholding for 1111 detection, and Gaussian fitting for precise estimation of subpixelic positions. Drift 1112 correction was performed computationally by tracking fluorescent beads used as 1113 fiducial markers. 


\section{Quality metrics and sampling resolution}

1116 In order to quantitatively assess the quality of PALM images and ANNA-PALM

1117 reconstructions, we calculated the multi-scale structural similarity index (MS-SSIM)

1118 between either image and the ground truth (Figure 2g and Supplementary

1119 Figures 5,9). For the simulated data, the ground truth was simply defined as the

1120 "perfect" PALM image, corresponding to an infinite number of localizations (see

1121 'Image simulations' above, Figure 2e and Supplementary Figure 1c). For the

1122 experimental data, the ground truth was defined as the ANNA-PALM reconstruction

1123 of a dense PALM image obtained from all available frames (e.g. Supplementary

1124 Figure 9c). Before calculation of the MS-SSIM, all simulated images were linearly

1125 normalized without clipping to a maximum value of 255.

1126 In order to evaluate the effect of sampling on the resolution of PALM images,

1127 we computed the double mean distance, $R_{\mathrm{Nyq}}=2\langle d\rangle$ between nearest neighbors in

1128 the underlying sets of localizations ${ }^{49}$ as function of localization number

1129 (Supplementary Figure 3d). For the simulated sparse PALM data, sets of

1130 localizations were obtained by interpreting each image $\boldsymbol{S}$ as a 2D histogram of

1131 localizations, and creating a random subpixelic position $\left(x_{k}, y_{k}\right)_{k=1 . . m}$ within each

1132 pixel $(i, j)$, as many times as given by the pixel value $m=S(i, j)$ (therefore resulting

1133 in a set of $n=\sum_{i, j} S(i, j)$ localizations). The quantity $R_{\mathrm{Nyq}}(n)=2\langle d(n)\rangle$ decreases

1134 towards zero with increasing number of localizations, $n$. A Nyquist criterion

1135 introduced in ref. ${ }^{49}$ suggests that the resolution is limited by sampling to no less

1136 than $R_{\mathrm{Nyq}}$, implying that at least $n \geq N_{\mathrm{Nyq}}=R_{\mathrm{Nyq}}^{-1}(R)$ localizations are needed to

1137 achieve a given resolution $R$. However, a more stringent and realistic criterion ${ }^{3}$ 
1138 prescribes a five-fold larger number of localizations to reach resolution $R$ :

$1139 n \geq N_{5 \times \mathrm{Nyq}}=5 \times N_{\mathrm{Nyq}}$, implying that the sampling limit to resolution is

$1140 R_{5 \times \mathrm{Nyq}}=2\langle d(n / 5)\rangle$. Accordingly, if this condition is met, the resolution is no longer

1141 limited by sampling, but by the localization precision, $R_{\mathrm{loc}} \approx 2.3 \sigma_{\mathrm{loc}}$, where $\sigma_{\mathrm{loc}}$ is

1142 the standard deviation of localization errors along each coordinate. In general, the

1143 resolution of a PALM image, as limited by both sampling and localization precision,

1144 can be written: $R=\max \left(R_{5 \times \mathrm{Nyq}}, R_{\mathrm{loc}}\right)=\max \left(2\langle d(n / 5)\rangle, 2.3 \sigma_{\mathrm{loc}}\right)$.

1145

1146 Life Sciences Reporting Summary.

1147 Further information is available in the Life Sciences Reporting Summary.

1149 Code availability statement

1150 The source code of ANNA-PALM is available from https://annapalm.pasteur.fr/.

\section{Data availability statement}

1153 The localization data used in this paper can be downloaded directly from

1154 https://annapalm.pasteur.fr/.

\section{Methods-only references}

1157 51. Xu, L., Choy, C. S. \& Li, Y. W. Deep sparse rectifier neural networks for speech

1158

1159 denoising. in 2016 International Workshop on Acoustic Signal Enhancement, IWAENC 2016 (2016). doi:10.1109/IWAENC.2016.7602891

1160

1161

1162

1163

52. Maas, A. L., Hannun, A. Y. \& Ng, A. Y. Rectifier Nonlinearities Improve Neural Network Acoustic Models. Proc. 30 th Int. Conf. Mach. Learn. (2013).

53. Mao, X. et al. Least Squares Generative Adversarial Networks. in ICCV 27942802 (2017). doi:10.1109/ICCV.2017.304 
1164 54. Kingma, D. P. \& Ba, J. Adam: A Method for Stochastic Optimization. ICLR 1-15

1165 (2015).

1166

1167 doi:http://doi.acm.org.ezproxy.lib.ucf.edu/10.1145/1830483.1830503

1168

55. Rampasek, L. \& Goldenberg, A. TensorFlow: Biology's Gateway to Deep Learning? Cell Syst. 2, 12-14 (2016).

1169

56. Srinivasa Reddy, B. \& Chatterji, B. N. An FFT-based technique for translation, rotation, and scale-invariant image registration. IEEE Trans. Image Process. 5, 1266-1271 (1996).

1171

1172

1173

1174

1175

1176

1177

1178

1179

1180

1181

1182

1183

1184

1185

1186

1187

1188

1189

1190

1191

1192

1193

1194

1195

57. Simard, P. Y., Steinkraus, D. \& Platt, J. C. Best practices for convolutional neural networks applied to visual document analysis. Seventh Int. Conf. Doc. Anal. Recognition, 2003. Proceedings. 1, 958-963 (2003).

58. Plimpton, S. Fast Parallel Algorithms for Short-Range Molecular Dynamics. J. Comput. Phys. 117, 1-19 (1995).

59. van de Linde, S. et al. Direct stochastic optical reconstruction microscopy with standard fluorescent probes. Nat. Protoc. 6, 991-1009 (2011).

60. Henriques, R. et al. QuickPALM: 3D real-time photoactivation nanoscopy image processing in ImageJ. Nat. Methods 7, 339-340 (2010).

61. Lelek, M. et al. Superresolution imaging of HIV in infected cells with FlAsHPALM. Proc. Natl. Acad. Sci. U. S. A. 109, 8564-9 (2012).

62. Edelstein, A., Amodaj, N., Hoover, K., Vale, R. \& Stuurman, N. Computer control of microscopes using manager. Current Protocols in Molecular Biology (2010). doi:10.1002/0471142727.mb1420s92

63. Ovesny, M., Kiek, P., Borkovec, J., Vindrych, Z. \& Hagen, G. M. ThunderSTORM: a comprehensive ImageJ plug-in for PALM and STORM data analysis and super-resolution imaging. Bioinformatics 30, 2389-2390 (2014).

64. Preibisch, S., Saalfeld, S. \& Tomancak, P. Globally optimal stitching of tiled 3D microscopic image acquisitions. Bioinformatics 25, 1463-1465 (2009).

65. Sergé, A., Bertaux, N., Rigneault, H. \& Marguet, D. Dynamic multiple-target tracing to probe spatiotemporal cartography of cell membranes. Nat Methods 5, 687-694 (2008). 

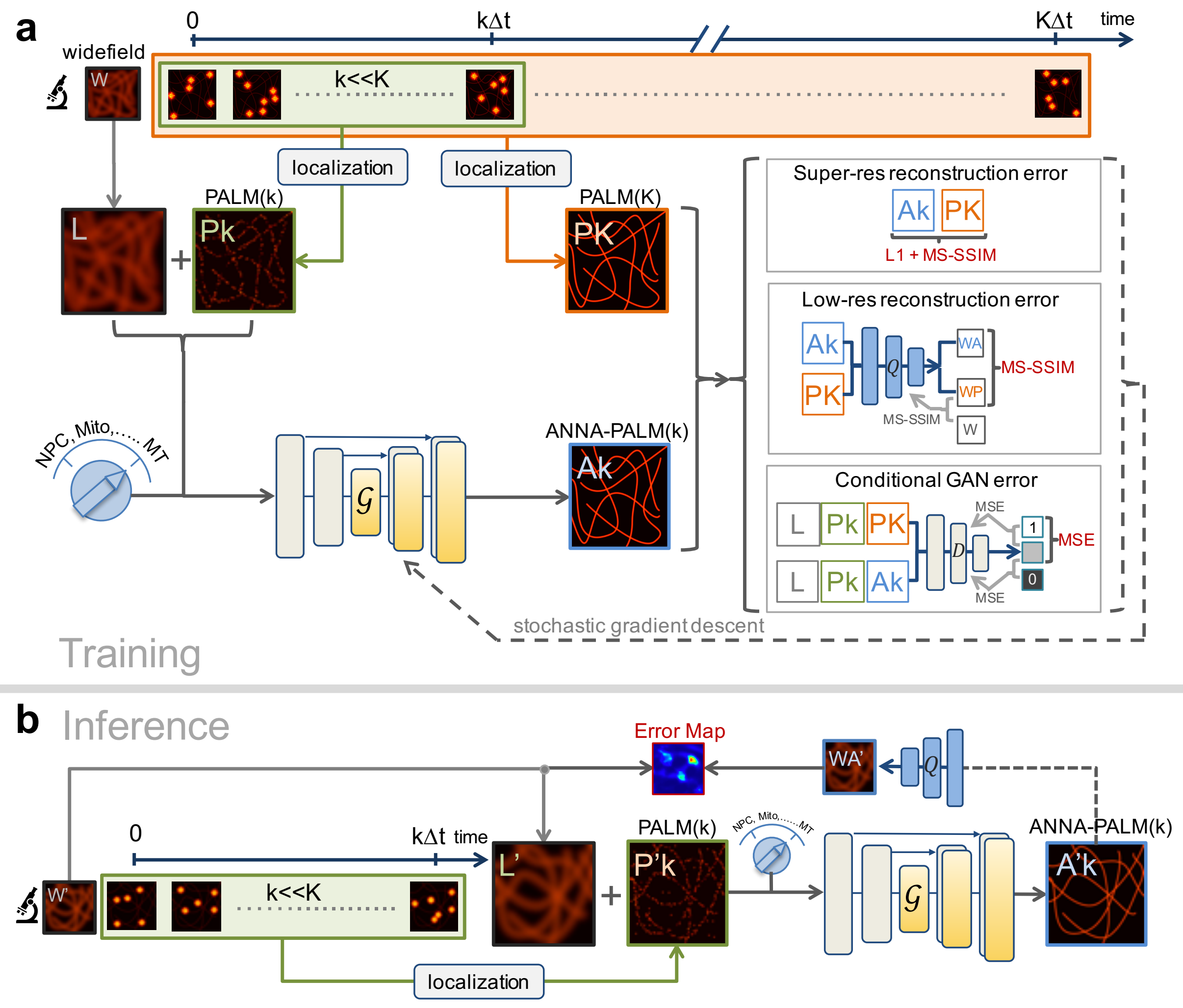

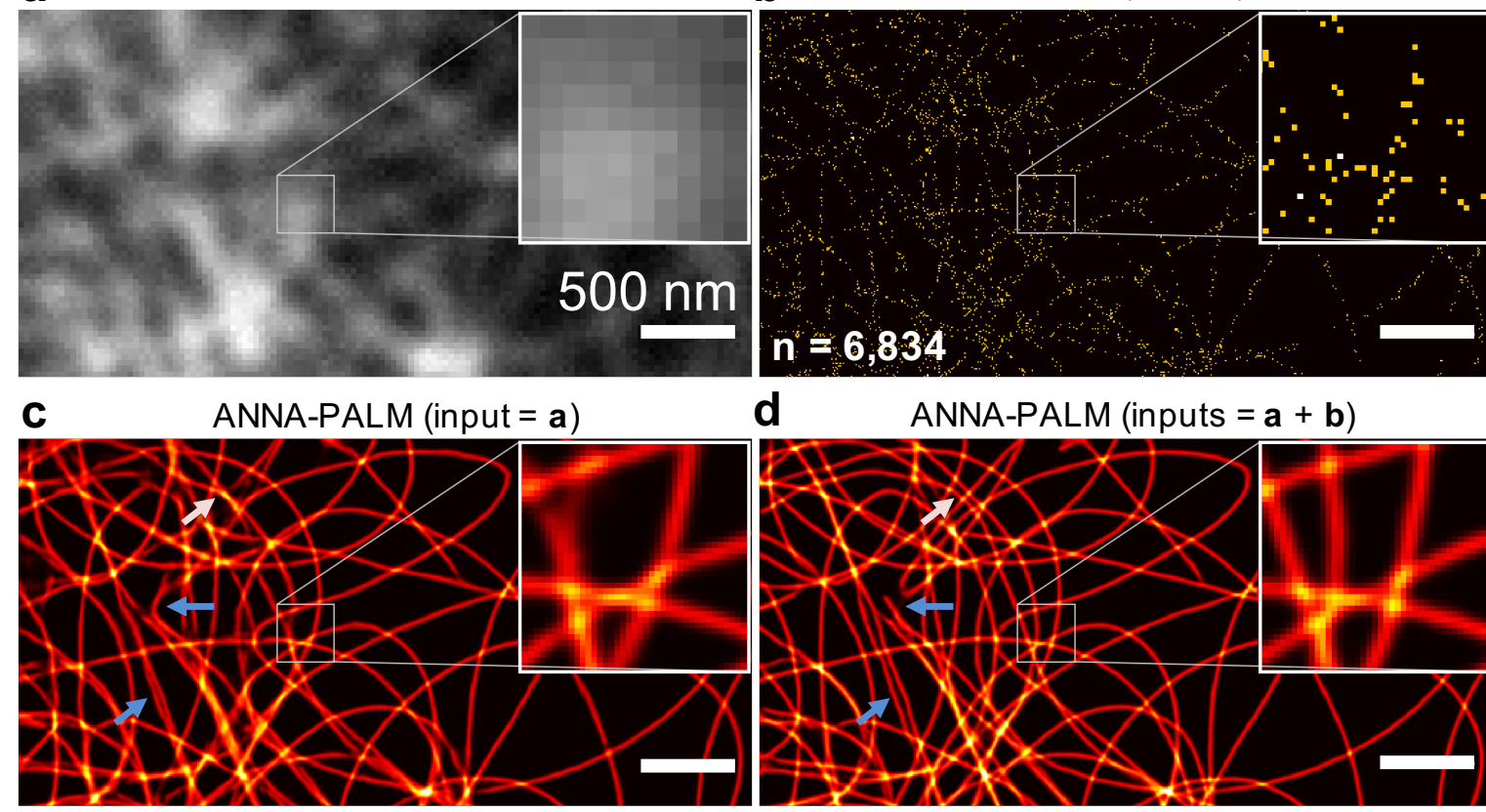

e

PALM (perfect)

$$
n=6,834
$$

d ANNA-PALM (inputs $=\mathbf{a}+\mathbf{b}$ )
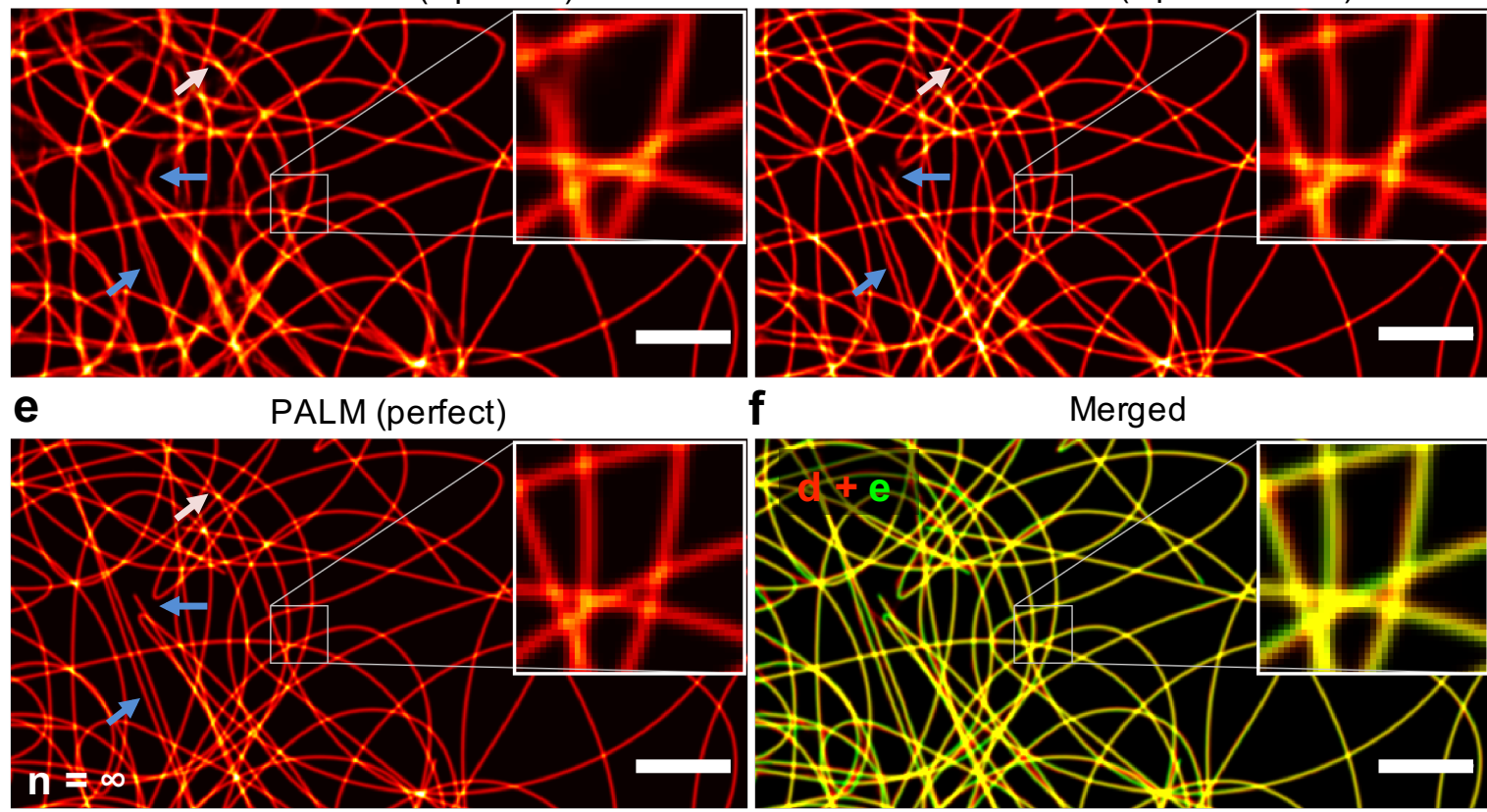

f

Merged

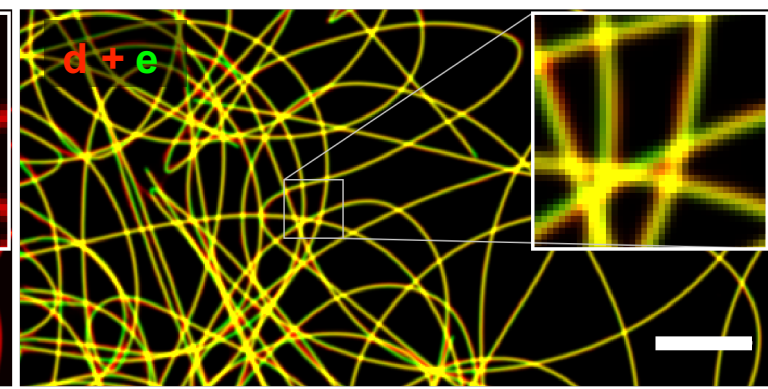

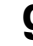

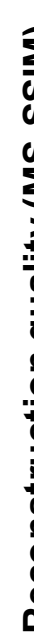

1x Nyquist

5x Nyquist
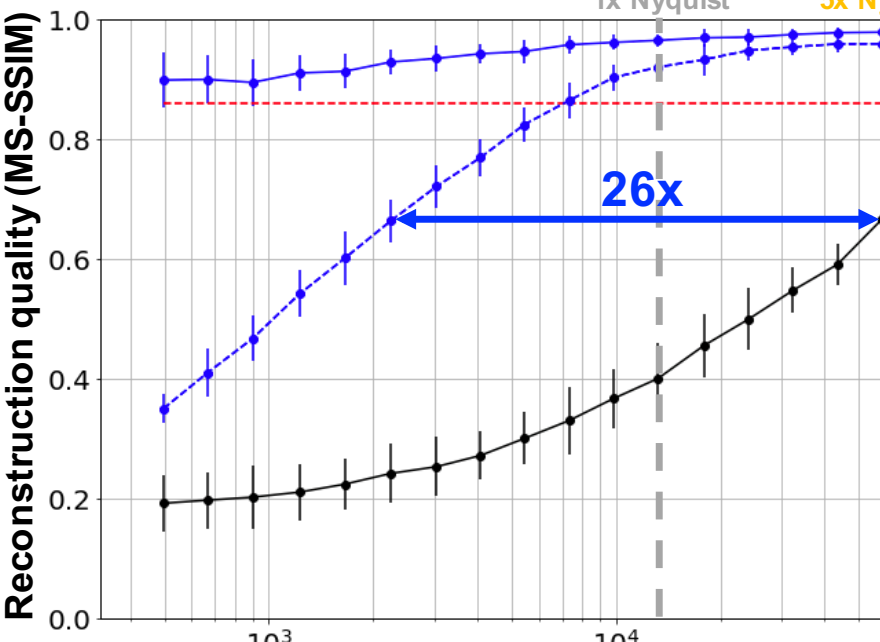

PALM

ANNA-PALM (sparse+widefield) ANNA-PALM (sparse)

ANNA-PALM (widefield) 


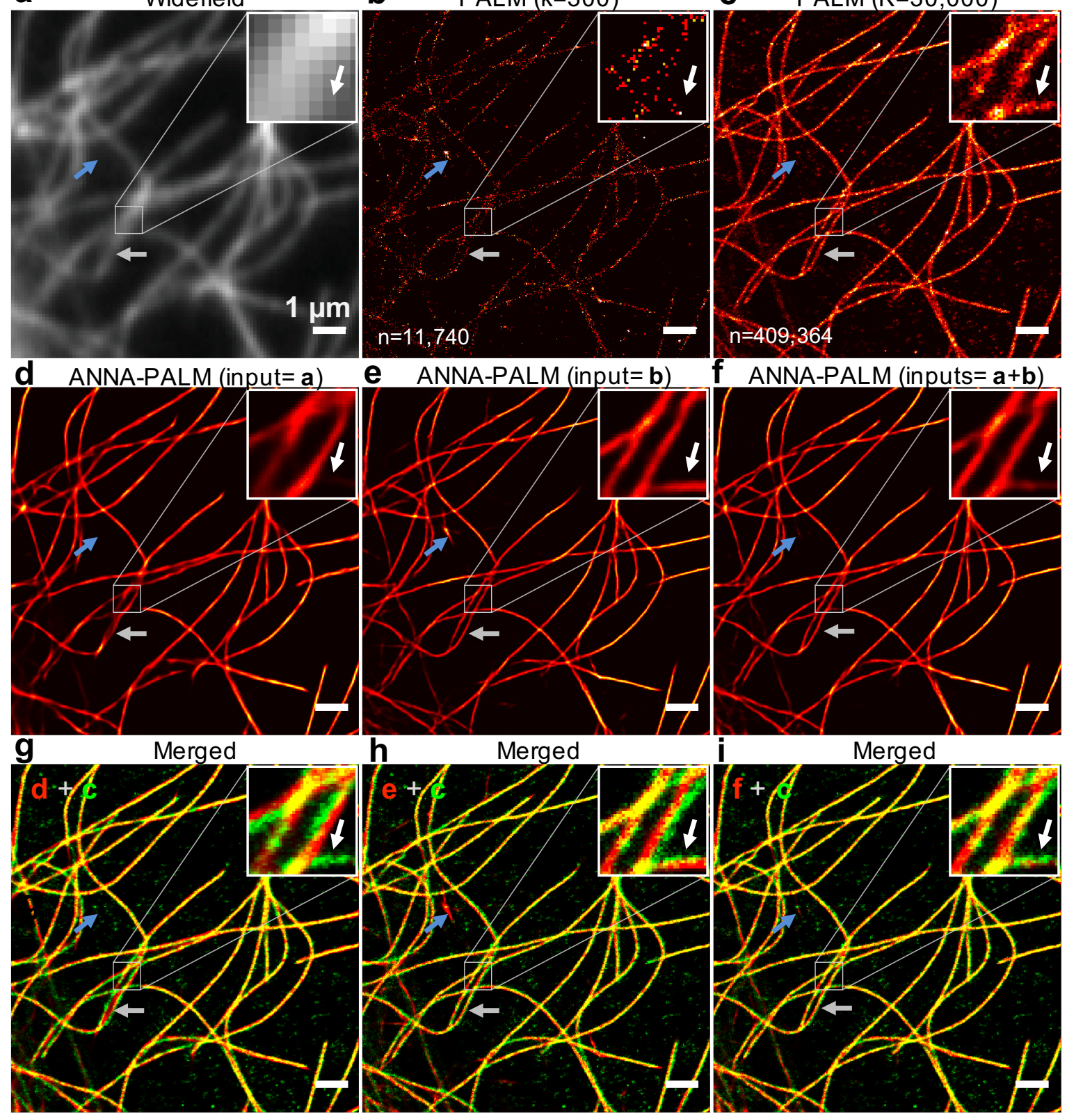

Vmin

$V_{\max }$

j.

10

40

$151 \mathrm{k} \Delta \mathrm{t}(\mathrm{s})$

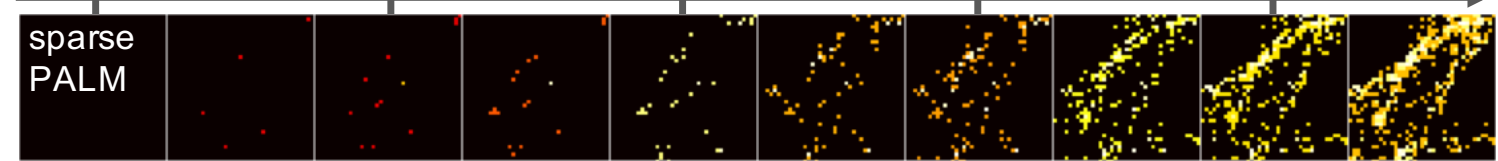

ANNA-

PALM

w/o WF 
PALM $(k=1000 \times 1089, t=3.1 \mathrm{~h})$

\section{a}

\section{ANNA-PALM $(\mathrm{t}=3.1 \mathrm{~h})$}

b

(

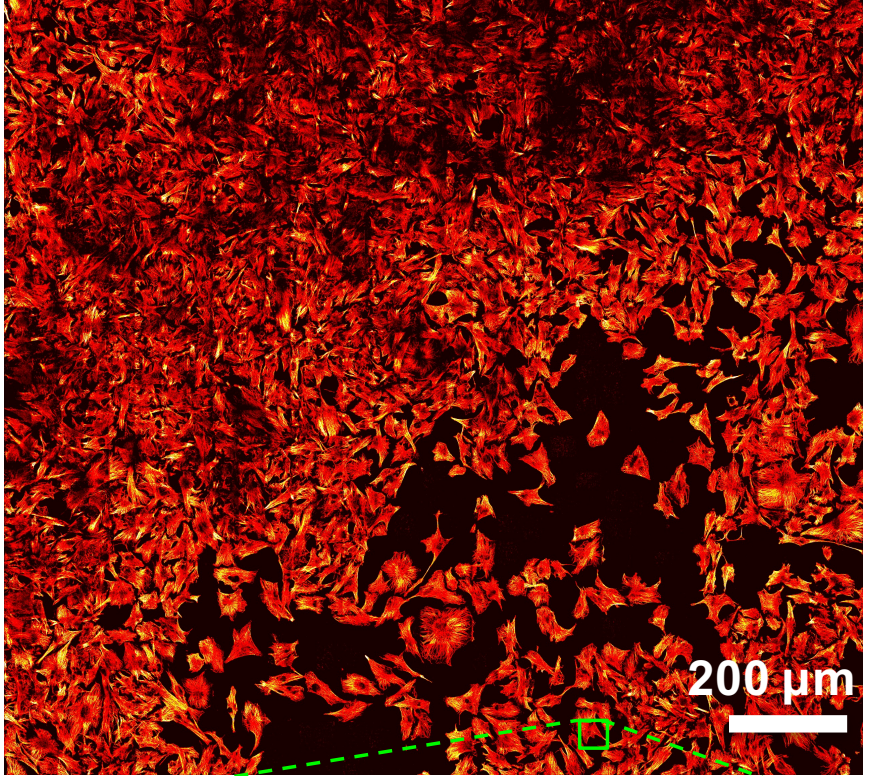

\section{C}

$10 \mu \mathrm{m}$
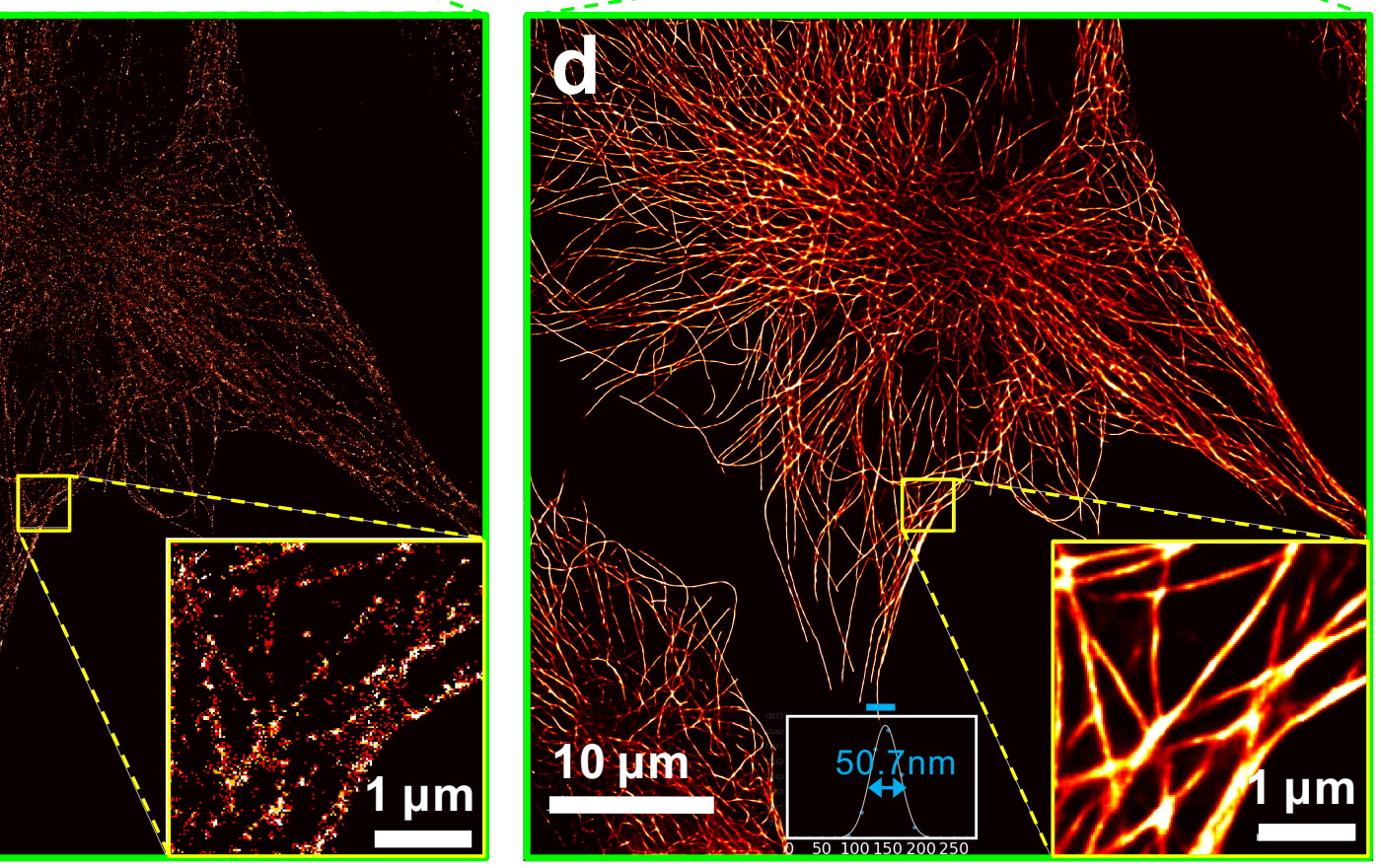


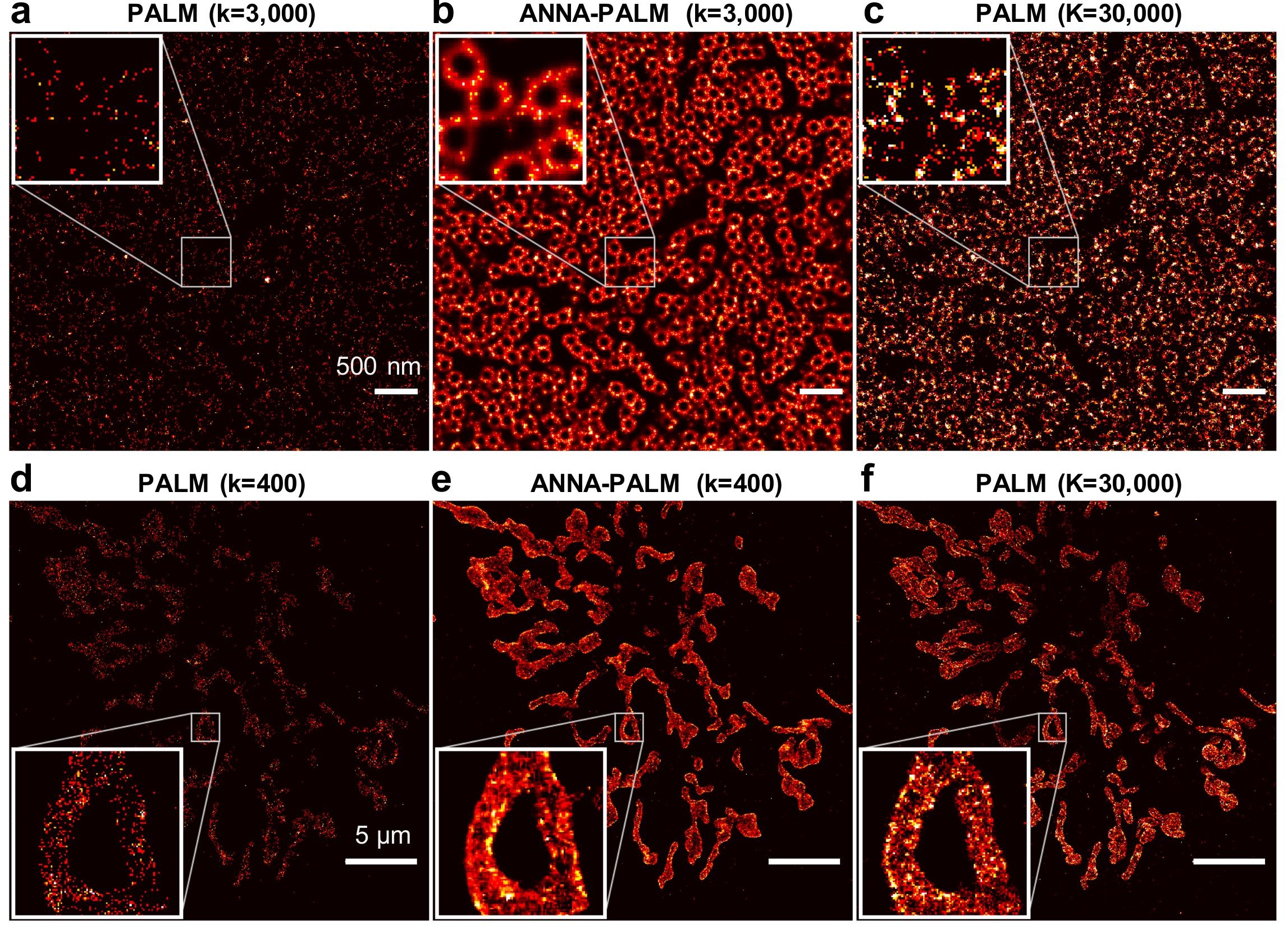

